\title{
THEORETICAL AND COMPUTATIONAL MODELING OF THE DYNAMICS OF MULTICELLULAR AND LIPID MEMBRANE SYSTEMS
}

A Dissertation presented to

the Faculty of the Graduate School

at the University of Missouri

In Partial Fulfillment

of the Requirements for the Degree

Doctor of Philosophy

by

MATTHEW MCCUNE

Dr. Ioan Kosztin, Dissertation Supervisor

DEC 2016 
(C) Copyright by Matthew McCune 2016

All Rights Reserved 
The undersigned, appointed by the Dean of the Graduate School, have examined the dissertation entitled:

\section{THEORETICAL AND COMPUTATIONAL MODELING OF THE DYNAMICS OF MULTICELLULAR AND LIPID MEMBRANE SYSTEMS}

presented by Matthew McCune, a candidate for the degree of Doctor of Philosophy and hereby certify that, in their opinion, it is worthy of acceptance.

Dr. Ioan Kosztin

Dr. Haskell Taub

Dr. Xiaoqin Zou

Dr. Carlos Wexler

Dr. Gavin King

Dr. Peter Cornish 


\section{DEDICATION}

I dedicate this dissertaiton to my wife, Sarah Lirley McCune, whose love and support over our many years together has given me the strength to continue to pursue my dreams and passion. 


\section{ACKNOWLEDGMENTS}

I would like to thank and express my appreciation first and foremost to my advisor, Dr. Ioan Kosztin, for his patience, advice, guidance, and mentorship throughout my graduate studies at the University of Missouri. Without his constant feedback and expertise my research projects would never have even gotten off the ground, much less completed. Special thanks also to Dr. Haskell Taub who went above and beyond the responsibilities of a committee member, meeting with me individually on numerous occasions to help me better understand how to advance my research and to better connect my simulations to experiment.

Thank you also to all of my committee members: Dr. Xiaoqin Zou, Dr. Carlos Wexler, Dr. Gavin King, Dr. Peter Cornish, and Dr. Taub, as well as past member Dr. Jason Cooley. Your comments and suggestions have been instrumental in the success of my research project. Finally, thank you to Dr. Jiong Zhang, Dr. Ashkan Shafiee, Dr. Andrew Miskowiec, Ph.D. candidate Zachary Buck, Dr. Flemming Hansen, and Dr. Gabor Forgacs for their helpful discussions, advice, and encouragement over the years.

*This work was supported in part by the National Science Foundation (NSF) [PHY0957914] and an NSF Integrative Graduate Education and Research Traineeship (DGE-1069091). The computations were performed on the HPC resources at the University of Missouri Bioinformatics Consortium (UMBC). 


\section{TABLE OF CONTENTS}

ACKNOWLEDGMENTS ............... ii

LIST OF TABLES $\ldots \ldots \ldots \ldots \ldots \ldots \ldots \ldots$

LIST OF FIGURES $\ldots \ldots \ldots \ldots \ldots \ldots$ vi

ABSTRACT .................... ix

\section{CHAPTER}

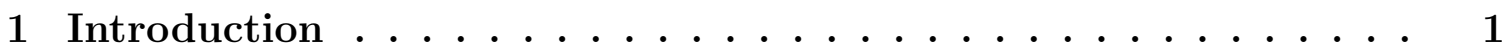

2 Modeling biomechanical relaxation processes of multi-cellular systems via Cellular Particle Dynamics . . . . . . . . . . . . 4

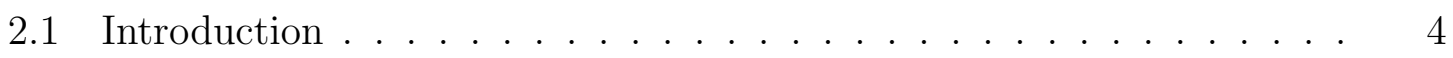

2.2 Methods . . . . . . . . . . . . . . . . . 6

2.3 General Theory . . . . . . . . . . . . . . . . . 9

2.4 Fusion of Spherical Aggregates . . . . . . . . . . . . . . . . 10

2.4 .1 Theory . . . . . . . . . . . . . . . . . . 10

2.4 .2 Calibration . . . . . . . . . . . . . . 16

2.4 .3 Validation . . . . . . . . . . . . . . . . . . . . 19

2.4 .4 Prediction . . . . . . . . . . . . . . . . 22

2.5 Fusion of Cylindrical Aggregates . . . . . . . . . . . . . . . . 24

2.5 .1 Theory . . . . . . . . . . . . . . . . . . 25

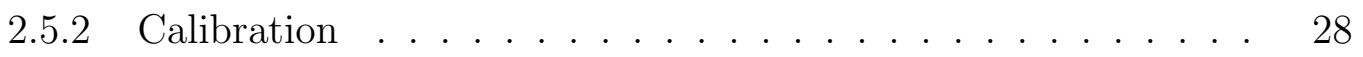

2.5 .3 Validation . . . . . . . . . . . . . . . . . . . 28 
2.5 .4 Prediction . . . . . . . . . . . . . . . . 32

2.5 .5 Application . . . . . . . . . . . . . . 33

2.6 Conclusion . . . . . . . . . . . . . . . . . . . . . . . . . . . . . 34

3 Structure and dynamics of lipid and water molecules in free-standing and supported hydrated phospholipid bilayer systems . . . . 36

3.1 Introduction . . . . . . . . . . . . . . . 36

3.2 Methods . . . . . . . . . . . . . . . . . . . . . 39

3.2 .1 Bilayer Simulations . . . . . . . . . . . . . . . . . 39

3.2.2 Annealing Simulation of the Silica Substrate . . . . . . . . 40

3.3 Results . . . . . . . . . . . . . . . . . . . . . . . 42

3.3.1 Bilayer: free-standing . . . . . . . . . . . . . . . . . 42

3.3 .2 Silica Substrate . . . . . . . . . . . . . . . . . . . 44

3.3 .3 Bilayer: supported . . . . . . . . . . . . . . . . . 4 44

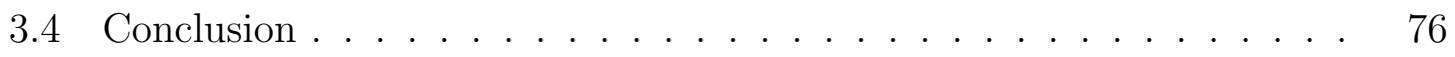

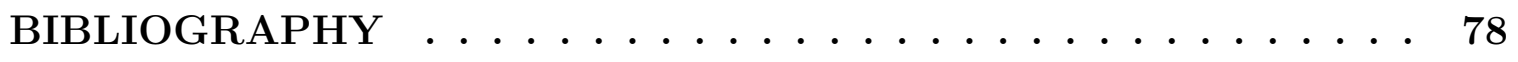

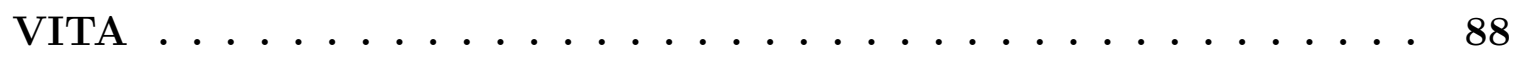




\section{LIST OF TABLES}

Table $\quad$ Page

2.1 CPD Simulation Parameter Values . . . . . . . . . . . . . . . . 9

2.2 Nomenclature - CPD simulations and EHAM experiments . . . . . 16

2.3 Fitting parameter values - CPD simulations and EHAM experiments 21

2.4 Nomenclature - CPD simulations and extrusion experiments . . . . . 28

2.5 Fitting parameter values - CPD simulations and extrusion experiments 31

3.1 Silica WCA force field parameter values . . . . . . . . . . . . . . 41

3.2 FS and SP Systems: Bilayer Thickness . . . . . . . . . . . . . 52

3.3 SP: Water Layer Thickness . . . . . . . . . . . . . . . . . . 54

3.4 Out-of-Plane Lipid Fluctuations . . . . . . . . . . . . . 58

3.5 Water Molecules per Lipid . . . . . . . . . . . . . 61

3.6 Lipid Phosphorus Lateral Diffusion Coefficients $(2 \leq t \leq 10 \mathrm{~ns})$. . . 68

3.7 Lipid Phosphorus Lateral Diffusion Coefficients (10 $\leq t \leq 50 \mathrm{~ns})$. . . 69

3.8 Water Layer Definitions I . . . . . . . . . . . . . . . . 70

3.9 Water Lateral Diffusion Coefficients . . . . . . . . . . . . 73

3.10 Water Layer Definitions II . . . . . . . . . . . . . . . . . 74 


\section{LIST OF FIGURES}

Figure $\quad$ Page

2.1 Schematic of multi-layered tube creation using spherical bioink . . . . 5

2.2 CPD simulation force field potentials . . . . . . . . . . . . . . 7

2.3 Contour of fusing aggregates . . . . . . . . . . . . . . . 11

2.4 Simulation result: fusion of even spherical aggregates . . . . . . . . 17

2.5 Experimental result: fusion of even spherical aggregates (EHAM) . . 18

2.6 Simulation result: fusion of uneven spherical aggregates . . . . . . . . 19

2.7 Experimental result: fusion of uneven spherical aggregates (EHAM) . 20

2.8 Volume change during fusion process for even and uneven spherical

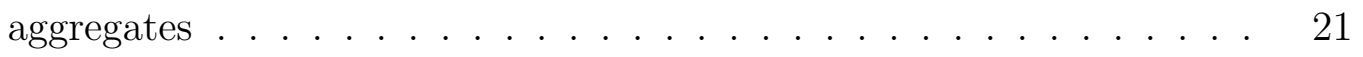

2.9 Simulation prediction: fusion of even spherical aggregates (EHAM) . 22

2.10 Simulation prediction: fusion of uneven spherical aggregates (EHAM) 24

2.11 Experimental result: fusion of even spherical aggregates (extrusion) . 29

2.12 Simulation result: fusion of even cylindrical aggregates . . . . . . . . 30

2.13 Experimental results: fusion of even cylindrical aggregates (extrusion) 31

2.14 Volume and cylinder length change during experimental fusion process for even spherical and cylindrical aggregates . . . . . . . . . . . . 32 
2.15 Simulation prediction: fusion of even cylindrical aggregates (extrusion) 34

2.16 Simulation results: tube creation using spherical and cylindrical aggre-

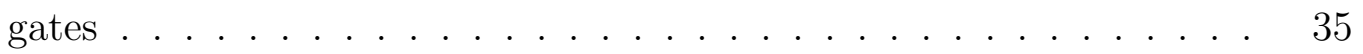

3.1 Schematic of a lipid bilayer mounted on a silica substrate . . . . . . . 38

3.2 Simulation snapshot: FS hydrated bilayer systems . . . . . . . . . . . 42

3.3 Simulation result: FS mass and number density profiles . . . . . . . . 43

3.4 Simulation result: water mass density profile on a silica substrate . . 44

3.5 Simulation snapshot: SP9 and SP14 hydrated bilayer systems . . . . 45

3.6 Simulation snapshot: SP14 hydrated bilayer systems (atomic represen-

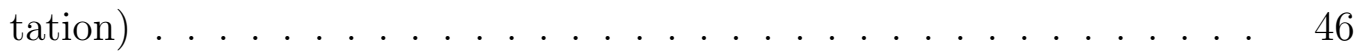

3.7 Simulation result: water hydration level thickness . . . . . . . . . . . 47

3.8 AFM experimental result: bilayer thickness . . . . . . . . . . . . . 49

3.9 Simulation result: bilayer thickness and mean $z$ position . . . . . . 51

3.10 Simulation result: acyl chain order parameter . . . . . . . . . . 53

3.11 Simulation result: hydrophilic layer thickness . . . . . . . . . . . 54

3.12 Simulation result: membrane surface tension . . . . . . . . . . 55

3.13 Simulation result: probability density, mean, standard deviation of lipid phosphorus $z$ coordinates . . . . . . . . . . . . . . 57

3.14 Simulation result: mass density profiles (water and all atoms) . . . . 59

3.15 Simulation result: water hydration about the lipid phosphorus atoms 61

3.16 Simulation result: water mean dipole orientation profile . . . . . . . . 62

3.17 Simulation result: mean orientation of lipid PN vector . . . . . . 63

3.18 Simulation result: lipid MSD - P, CH, and CT . . . . . . . 65

3.19 Simulation result: lipid MSD - phosphorus . . . . . . . . . . 67 
3.20 Water layer snapshots . . . . . . . . . . . . . . . . 71

3.21 Simulation result: water MSD and $N / N_{0}$ (layer definitions I) . . . . 72

3.22 Simulation result: water MSD and $N / N_{0}$ (layer definition II) . . . . . 75 


\begin{abstract}
This dissertation presents two research projects that apply theoretical and computational modeling to (1) describe and predict the formation and shape evolution of three-dimensional (3D) bioprinted tissue constructs, and (2) investigate the effect of a silica substrate on the structural and dynamic properties of a single fully hydrated lipid bilayer.

(1) Bioprinting, a novel tissue engineering technique, has the ultimate goal of using 3D printers with bioink made from a person's own cells to create tissues in the laboratory for transplantation or drug testing. The outcome of the post-bioprinting process, where the bioink particles fuse to form the desired 3D tissue construct, is difficult to predict and experimental techniques have generally been optimized through trial and error. To address this shortcoming, by employing theoretical modeling and computer simulations, we have developed and implemented an effective procedure that is capable of describing and predicting the shape dynamics during post-printing structure formation in 3D bioprinting. In particular, we have explained and demonstrated that the post-printing fusion process is considerably faster when using cylindrical instead of spheroidal bioink particles, a result that has considerable practical implication for extrusion bioprinting.

(2) The study of lipid bilayers using neutron scattering experiments requires samples that contain a large stack of membranes. The analysis and computer simulation of such systems is challenging mainly due to the unknown amount of water separating the membranes. To overcome this difficulty, more recent experiments place single lipid membranes onto a support and stack about a hundred of them together.
\end{abstract}


In this project we use molecular dynamics simulations of both free-standing and hydrated single-supported lipid bilayers to investigate the effect of the silica substrate on the structural and dynamical properties of the lipids and hydration waters. Our results may provide useful information in interpreting some recent neutron scattering experiments. 


\section{Chapter 1}

\section{Introduction}

This thesis presents two research projects that applies theoretical and computational modeling to (1) describe and predict the formation and shape evolution of threedimensional (3D) bioprinted tissue constructs, and (2) investigate the effect of a silica substrate on the structural and dynamic properties of a single fully hydrated lipid bilayer.

The first project (Ch. II) is a collaboration between our theoretical and computational group and the experimental bioprinting lab of Dr. Gabor Forgacs. Bioprinting, a novel tissue engineering technique, has the ultimate goal of creating tissues in the lab for transplantation or drug testing [1-4]. This experimental technique employs a 3D printer which places, through layer-by-layer deposition of bioink, an initial grouping of multi-cellular aggregates which will fuse to create the final desired shape [5-7]. Through this collaboration our groups have developed Cellular Particle Dynamics (CPD), a theoretical-computational-experimental framework that can be used to predict the time evolution, final shapes, and characteristic fusion times that 
multi-cellular aggregates will take in vitro to help guide tissue fusion experiments.

Previous work has demonstrated the ability of CPD simulations in describing the fusion of identical aggregates in vitro $[8,9]$. Continuum models were derived that quantitatively described the dynamics for the fusion of identical spheres and cylinders. These models assumed that the volume of the spherical aggregates was conserved, which is true in simulation but rarely in experiment. This work extends the $\mathrm{CPD}$ theory to describe experiments where the volume of the aggregates changes in time [10]. Using cylindrical bioink introduces additional complexity since the lengths of the cylindrical aggregates will contract postprinting. We have run simulations and developed theoretical models which can account for both volume loss and length contraction of cylinders while undergoing fusion [11]. Additionally, we have shown how the theoretical models can be used as an intermediary to connect simulation and experiment, and how predictions can be made directly from simulations.

The second project (Ch. III) is a collaboration with the neutron scattering group of Dr. Haskell Taub. Their experimental group performs neutron scattering on hydrated lipid bilayers mounted on silica substrates to determine structural and dynamical properties of water layers on the bilayer $[12,13]$. Our group has used molecular dynamics to study free-standing bilayers in the past but here an amorphous silica substrate is introduced below the bilayer to see how the presence of the substrate affects certain structural and dynamical properties of the lipid bilayer and the hydration layers $[14,15]$.

Specifically the properties of interest, which were measured and compared to experiment when possible, were the (i) thickness of the hydration layer between the bilayer and silica, (ii) hydrophilic layer thickness, (iii) bilayer thickness, (iv) acyl chain 
order parameter, (iv) membrane surface tension, (v) out-of-plane lipid fluctuations, (vi) mass and number density, (vii) mean water dipole orientation, (viii) phospholipid PN vector orientation, (ix) mean squared displacement and diffusion coefficients for the lipids and water, and (x) radial distributions for the water molecules about the lipid headgroups. 


\section{Chapter 2}

\section{Modeling biomechanical relaxation processes of multi-cellular systems via Cellular Particle Dynamics}

\section{$2.1 \quad$ Introduction}

In the post-printing phase of bioprinting, the bioink, which are multi-cellular aggregates composed of thousands of cells, will relax (i.e., fuse) to form the final continuous biological structure. A quantitative description of the process is difficult and experimental techniques have generally been optimized through trial and error. Here we will demonstrate using Cellular Particle Dynamics (CPD) $[8,9]$ how the fusion process, which follows physical laws, can be modeled quantitatively to address the lack of predictability of the fusion process for simple systems composed of two fusing aggregates and even complex structures with many aggregates.

Previous work has demonstrated the ability of the CPD model to describe the 

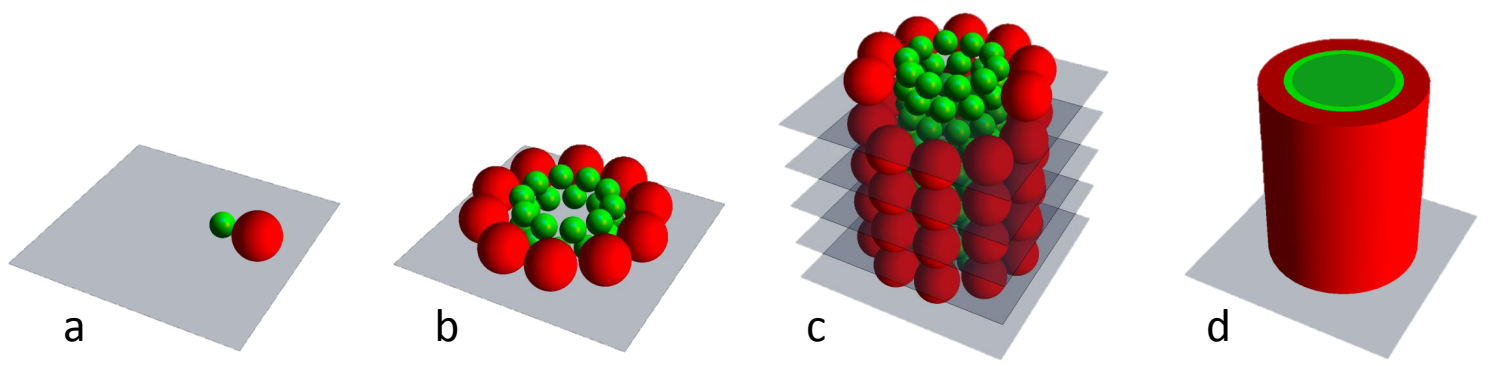

Figure 2.1: Schematic showing the process for printing (a-c) a tube with nonidentical bioink particle sizes and the final fused shape (d). The simple case of two fusing uneven multicellular aggregates (a) of the same cell type is the focus of Section 2.4.

fusion of two identical spherical and cylindrical aggregates, as well as the fusion of a ring of ten identical spherical aggregates [9], but only for the simplest case where the volume of the system and cylinder lengths are assumed to be conserved, which conflicts with what has been observed in experiment. Also, bioprinting experiments do not exclusively use aggregates with identical initial radii, as nonidentical aggregates are necessary to build a tube where only a small layer of cells are wanted in the tube's interior, e.g., a primitive blood vessel of smooth smooth muscle cells with the interior lined with endothelial cells, Fig. 2.1.

My research has (i) extended the CPD model to a fusing system where the interacting multi-cellular spherical aggregates do not have the same initial radii [10], (ii) extended the theoretical models to account for volume decrease (and cylinder length contraction) for fusing spherical (cylindrical) aggregates [11], and (iii) made experimental predictions directly from the CPD simulations thus eliminating the need for the intermediary theory. 


\subsection{Methods}

Within the CPD framework, cells are coarse-grained into cellular particles (CPs), with attractive and repulsive interactions optimized so the multi-cellular aggregates behave similarly to experiment. CPs in different cells interact via strong but shortrange forces that mimic cell contact interactions, while CPs within the same cell have an additional confining force which keeps the CPs together, acting as an effective cell membrane. After the summation of these inter- and intra-molecular forces along with an additional stochastic force (accounting for the stochastic environment inside the cell), the equation of motion for each $\mathrm{CP}$ is time integrated to determine its trajectory. The trajectories of the CPs are recorded and used to extract the time evolution of the shape of the multi-cellular system.

From experiment, it is known that a single multi-cellular aggregate of any shape will round to form a sphere to minimize its surface energy. Also, when two spherical (cylindrical) aggregates are brought into contact they will fuse as spherical caps (truncated cylinders), forming a single larger aggregate. During this process, very little cell mixing is observed between the two multi-cellular aggregates. This behavior is typical of highly viscous liquids, hence continuum theory for viscoelastic liquids is used to derive a theoretical model for the dynamics of the fusion of multi-cellular aggregates. Furthermore, CPD simulation parameter values have been chosen to produce simulations with the same aggregate behavior as in experiments.

While an individual cell within a multi-cellular aggregate may have a defined volume, its shape is not rigid. This shape-change behavior (i.e., cell surface fluctuations) has been shown to increase the cell's motility [16,17]. To mimic a cell with a dynamic shape in CPD, each cell has been coarse-grained into ten cellular particles where each 
(a)

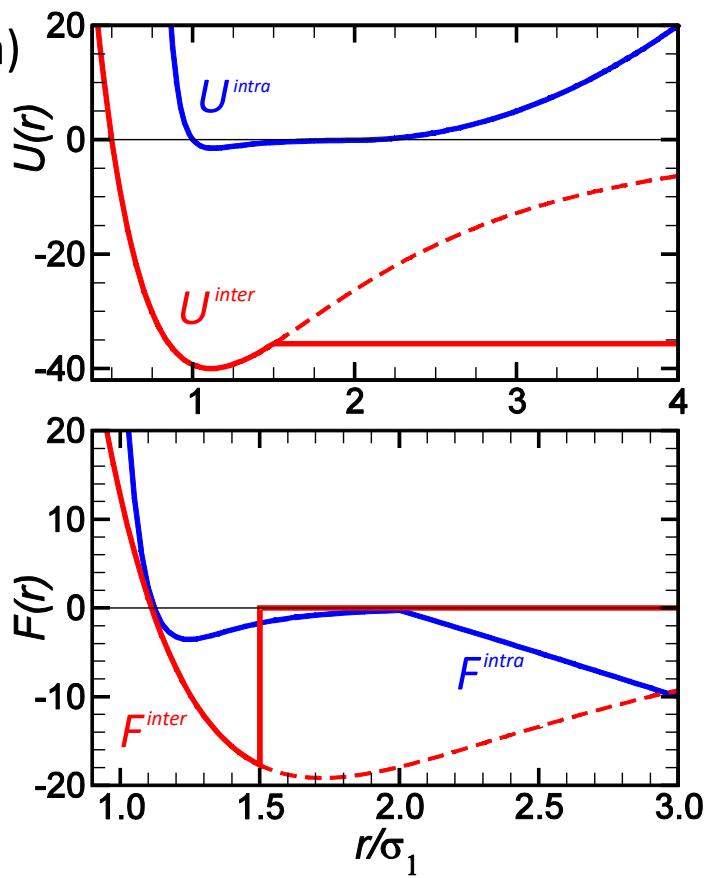

(b)

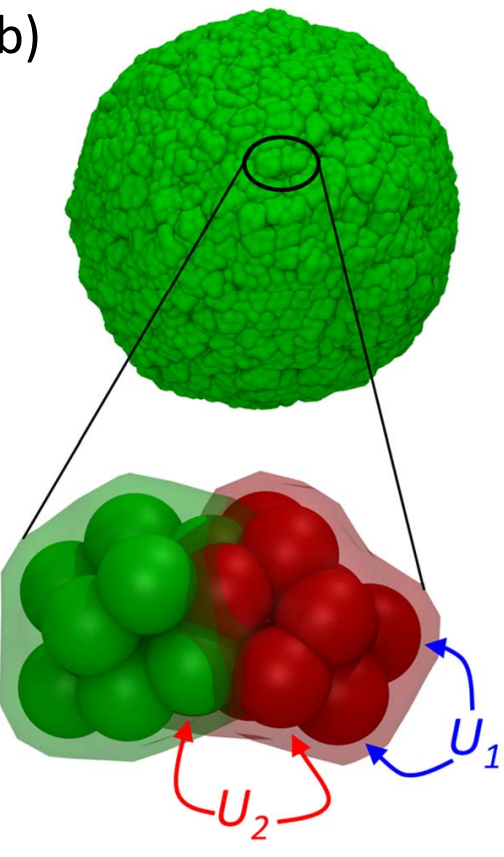

Figure 2.2: (a) Interaction potentials (top) and forces (bottom) between CPs in the same cell (blue) and different cells (red). (b) The image shows a 2,000 cell aggregate and two contacting cells with representative interactions drawn between a few CPs.

$\mathrm{CP}$ represents a volume element of the original cell. A CP can interact with other CPs within the same cell (intracellular) and with CPs in different cells (intercellular). The potential energies corresponding to these interactions are are given by

$$
\begin{aligned}
U= & U^{\text {inter }}+U^{\text {intra }} \\
U^{\text {inter }}(r)= & V_{L J}\left(r ; \epsilon^{\text {inter }}, \sigma^{\text {inter }}, \delta\right) ; r<r_{c} \\
U^{\text {intra }}(r)= & V_{L J}\left(r ; \epsilon^{\text {intra }}, \sigma^{\text {intra }}, 0\right) \\
& +\frac{k}{2}(r-\xi)^{2} \Theta(r-\xi)
\end{aligned}
$$


where

$$
V_{L J}(r ; \epsilon, \sigma, \delta)=4 \epsilon\left[\left(\frac{\sigma}{r+\delta}\right)^{12}-\left(\frac{\sigma}{r+\delta}\right)^{6}\right]
$$

Both potential energies have an attractive and a repulsive region, defined through the generalized Lennard-Jones potential, Eq. (2.1c). Interactions between CPs in the same cell have an additional confining harmonic potential, the second term in Eq. (2.1b), which turns on only when two CPs in the same cell are farther away than the effective size of a cell, $\xi$. This keeps CPs in the same cell together, ensuring the integrity of the cell. CPs in different cells interact through strong, but short-range, forces that mimic cell adhesion, Eq. (2.1a). Since this interaction represents cell-tocell contact forces it is only present when the distance between two CPs is smaller than $1.5 \sigma^{\text {intra }}$, where $\sigma^{\text {intra }}$ is the diameter of a CP, Fig. 2.2.

Since multi-cellular aggregate systems behave similarly to highly viscous liquids, the motion of every $\mathrm{CP}$ is described by the overdamped Langevin equation

$$
\mu \dot{\boldsymbol{r}}(t)=-\nabla U+\boldsymbol{f}(t)
$$

where $\mu$ is the friction coefficient, $\boldsymbol{r}(t)$ is the position vector, $\boldsymbol{f}(t)$ is the random Langevin force, and the time derivative is denoted by the dot. The Langevin force is a Gaussian white noise with zero mean and variance $\left\langle f_{i}(t) f_{j}(0)\right\rangle=2 D \mu^{2} \delta(t) \delta_{i j}$, where $D$ is the effective diffusion coefficient of the CPs.

The CPD parameter values in Tab. 2.1 were chosen based on extensive simulations so the aggregates in simulation behave similarly to experiment, i.e., the aggregates fuse as spherical caps (or truncated cylinders) and minimal cell mixing occurs between aggregates during the fusion process. A more detailed description of how the specific 
values were chosen has been described previously $[8,9]$. We define the computer (i.e., CPD) length, time and energy units as,

$$
L=\sigma^{i n t r a}, \quad T=\frac{\left(\sigma^{i n t r a}\right)^{2}}{D}, \quad E=E_{T}=\mu D
$$

where $E_{T}$ is referred to as the "biological fluctuation energy" [18]. The CPD parameter values in Tab. 2.1 are pure numbers in these units, and $\sigma^{\text {intra }}=D=\mu=1$. In all simulations, the potentials, parameter values, and integrator were implemented within the molecular dynamics software LAMMPS [19].

\section{Table 2.1: CPD Simulation Parameter Values}

\begin{tabular}{lcc}
\hline \hline Property & Symbol & Value \\
\hline Elastic constant & $\mathrm{k}$ & 10 \\
Diameter of a cell & $\xi$ & 2.0 \\
Depth of LJ potential for CPs in same cell & $\epsilon^{\text {intra }}$ & 1.48 \\
Diameter of a CP & $\sigma^{\text {intra }}$ & 1.0 \\
Depth of LJ potential for CPs in different cells & $\epsilon^{\text {inter }}$ & 40.0 \\
Sigma value for cell-cell interactions & $\sigma^{\text {inter }}$ & 5.0 \\
Shift coefficient & $\delta$ & 4.5 \\
Cutoff distance & $r_{c}$ & 1.5 \\
\hline
\end{tabular}

\subsection{General Theory}

Experiments have shown that the soft tissue bioink behaves like highly viscous complex liquids and that throughout the fusion process the spheroidal particles fuse as spherical caps. This geometrical simplicity allows us to derive an analytical model that uses only mass and energy conservation, similar to the method used to describe how highly viscous molten drops fuse $[20,21]$. 
During the postprinting phase of bioprinting, the tissue fusion process is driven by the surface tension and resisted by the viscosity of the system. Hence, energy conservation allows us to equate the rate of loss of surface energy with the rate of energy dissipation due to viscosity,

$$
\begin{aligned}
& \frac{d W_{s}}{d t}=\frac{d W_{\eta}}{d t} \\
& \gamma \frac{d S}{d t}=-2 \eta \int\left(v_{n m}^{2}\right) d V
\end{aligned}
$$

where $\gamma$ is the surface tension coefficient, $S$ is the free surface area, $\eta$ is the viscosity coefficient, $v_{n m}$ is the velocity gradient tensor, and $V$ is the volume. For each system, fusing spherical or cylindrical aggregates, the free surface area and volume can be determined, both with and without volume and cylinder length conservation, and inserted into Eq. 2.5 to determine an equation that provides the time evolution of the radii $R$ and contact angle $\theta$, Fig. 2.3.

\subsection{Fusion of Spherical Aggregates}

\subsubsection{Theory}

During the fusion process of two non-identical (uneven) spherical aggregates, we define the ratio of the initial radii, $R_{i 0}$ for aggregate $i=1,2$, at $t=t_{0}$, the time at which the fusion process begins,

$$
b=\frac{R_{10}}{R_{20}}>1
$$




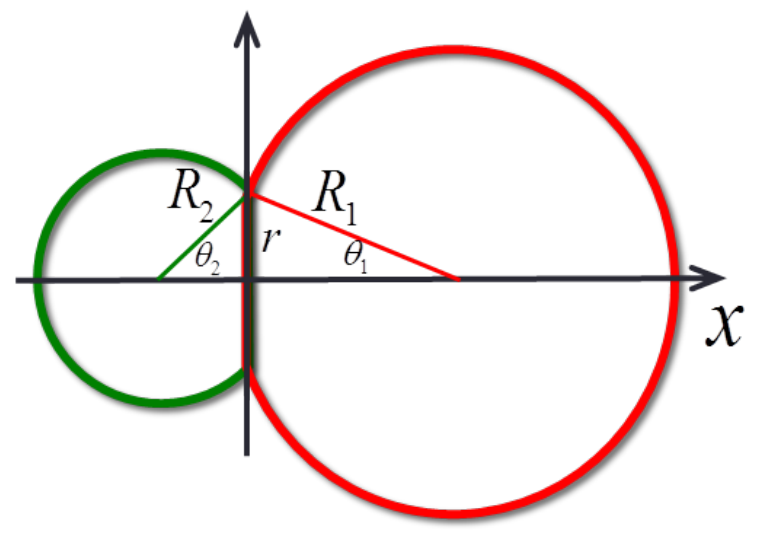

Figure 2.3: The outline (cross section) of two fusing uneven spherical (cylindrical) multi-cellular aggregates throughout the fusion process can be expressed as a function of the radius and contact angle for each aggregate: $R_{1,2}$ and $\theta_{1,2}$, for the larger and smaller aggregate, respectively. The length, $L$, is also needed for cylindrical fusion to fully describe the shape of the system. Even fusing aggregates is the special case where the two fusing spheres or cylinders have the same initial radii, $R_{10} \equiv R_{20}$ leading to $R_{1} \equiv R_{2}$ and $\theta_{1} \equiv \theta_{2}$.

The circular contact region between the aggregates during the fusion process has radius $r$,

$$
r=R_{1} \sin \theta_{1}=R_{2} \sin \theta_{2}
$$

as shown in Fig. 2.3. When the volume of the spherical caps does not change during the fusion process, the radius $R_{i}$ is entirely determined by the contact angle $\theta_{i}$ once the initial radii have been measured,

$$
\rho_{0 s}\left(\theta_{i}\right) \equiv \frac{R_{i}}{R_{i 0}}=2^{2 / 3}\left(1+\cos \theta_{i}\right)^{-2 / 3}\left(2-\cos \theta_{i}\right)^{-1 / 3}, i=1,2
$$

The naught in $\rho_{0 s}$ signifies that this is for the volume conserving case.

$$
R_{i}=R_{i 0} \cdot \rho_{0 s}\left(\theta_{i}\right)
$$


Inserting Eq. 2.9 into Eq. 2.7 we can determine either contact angle as a function of the other. The limits of the contact angles are $\theta_{1}\left(t_{0}\right)=\theta_{2}\left(t_{0}\right)=0$, and $\theta_{1}(\infty)=\theta_{\max }$ and $\theta_{2}(\infty)=\pi-\theta_{\max }$, where $\theta_{\max }$ depends on the initial radii ratio, $b$. For our system, where $b>1, \theta_{\max }<\pi / 2$.

The free surface area, initially $S_{10}+S_{20}$, where $S_{i 0}=4 \pi R_{i 0}^{2}$, can be defined as a function of the contact angles,

$$
S=S_{1}+S_{2}=S_{10} f_{1}+S_{20} f_{2}
$$

where

$$
f_{i} \equiv f\left(\theta_{i}\right)=\rho_{0 s}\left(\theta_{i}\right) \frac{1+\cos \theta_{i}}{2}
$$

Assuming volume conservation, the volume at all times is

$$
V_{i}=V_{i 0}=S_{i 0} R_{i 0} / 3
$$

Assuming a uniform biaxial extensional flow $[20,21]$, the continuity equation, $\operatorname{Tr}\left\{v_{n m}^{(i)}\right\}=$ 0 , yields

$$
\left[v_{n m}^{(i)}\right]^{2}=\frac{3}{2} u_{i}^{2}, \quad \text { for } i=1,2
$$

Inserting these equations into the energy balance equation, Eq. 2.5, results in

$$
\gamma\left(S_{10} f_{1}^{\prime} \dot{\theta}_{1}+S_{20} f_{2}^{\prime} \dot{\theta}_{2}\right)=-\eta\left(u_{1}^{2} S_{10} R_{10}+u_{2}^{2} S_{20} R_{20}\right)
$$


where $f_{i}^{\prime}=d f\left(\theta_{i}\right) / d \theta_{i}$. By employing the approximation $[8,9]$

$$
u_{i} \approx \frac{v_{x}^{(i)}}{R_{i}}=-\frac{1}{R_{i}} \frac{d\left(R_{i} \cos \theta_{i}\right)}{d t} \equiv g_{i} \dot{\theta}_{i}
$$

where

$$
g_{i} \equiv g\left(\theta_{i}\right)=\frac{1}{\rho_{0 s}\left(\theta_{i}\right)} \frac{d\left[\rho_{0 s}\left(\theta_{i}\right) \cos \theta_{i}\right]}{d \theta_{i}}
$$

after some algebra one obtains

$$
I_{1} \equiv I\left(\theta_{1}\right)=-\int_{0}^{\theta_{1}} \frac{b^{2} g_{1}^{2}+b^{-1} g_{2}^{2} h^{2}}{b^{2} f_{1}^{\prime}+f_{2}^{\prime} h} d \theta_{1}=t / \tau_{s}
$$

where $h \equiv h\left(\theta_{1}\right)=d \theta_{2} / d \theta_{1}$, and $\tau_{s}$ is the characteristic fusion time, which depends on the material constants $\eta$ and $\gamma$, and the initial size of the system,

$$
\tau_{s}=(\eta / \gamma) R_{10}
$$

Finally, we can determine the time dependence of the larger contact angle, $\theta_{1}$, for uneven spherical aggregate fusion by numerically integrating Eq. 2.17.

It was found that in experiments [10], the volume of the system decreases according to $V(t) / V_{0}=a^{3}(t)$, where

$$
a(t)=a_{0}+\left(1-a_{0}\right) \exp \left(-\lambda_{a} t\right)
$$

with $a_{0}=a(t=\infty) \leq 1$ and $\lambda_{a}^{-1}$ is the characteristic volume relaxation time. The change in volume affects the linear size of the system and the rate at which the fusion occurs but does not change the geometry of the fusing aggregates. 
When the volume changes, the radius is given by

$$
R_{i}=R_{i 0} \rho_{s}\left(\theta_{i}\right)
$$

where

$$
\rho_{s}\left(\theta_{i}\right)=\rho_{0 s}\left(\theta_{i}\right) a(t)
$$

We can approximate $\theta_{1}(t)$, taking into account the volume decrease, by replacing $R_{i 0}$ with $a(t) R_{i 0}$ and $S_{i 0}$ with $a^{2}(t) S_{i 0}$ in Eq. 2.14. Similarly to the derivation of Eq. 2.17, one obtains

$$
I\left(\theta_{1}\right)=\frac{1}{\tau_{s}} \int_{0}^{t} \frac{d t^{\prime}}{a\left(t^{\prime}\right)}
$$

where the right hand side of the equation can be directly integrated,

$$
I\left(\theta_{1}\right)=\left(\tau_{s} \lambda_{a} a_{0}\right)^{-1} \ln \left[1+a_{0}\left(\exp \left(\lambda_{a} t\right)-1\right)\right]
$$

Historically, in experiments the radius of the neck region, $r$, and the instantaneous radii, $R_{i}$, were measured and displayed as the ratio $\left(r / R_{i}\right)^{2}=\sin ^{2}\left(\theta_{i}\right)$. Following this procedure our results are displayed for both the volume conserving theory (Eq. 2.17; the naught in $q_{0 s}$ identifies the volume conserving case),

$$
q_{0 s} \equiv q_{0 s}\left(t / \tau_{s}^{*}\right)=\sin ^{2}\left(\theta_{i}\right)
$$

and the volume change theory (Eq. $2.22-2.23)$,

$$
q_{s} \equiv q_{s}\left(t / \tau_{s} ; a_{0}, \lambda_{a} \tau_{s}\right)=\sin ^{2}\left(\theta_{i}\right)
$$


One can show that $q_{0 s}$ can well approximate $q_{s}$ provided that one uses an effective fusion time

$$
\tau_{s}^{*}=c_{a} \tau_{s}
$$

where $c_{a} \approx\left(1+a_{0}\right) / 2<1[10]$. This means that the volume conserving theory can be used for experiments even when the volume does change, i.e., $q_{0 s}\left(t / \tau_{s}^{*}\right) \approx$ $q_{s}\left(t / \tau_{s} ; a_{0}, \lambda_{a} \tau_{s}\right)$. This implies that (i) a decrease in volume of the fusing aggregates speeds up the fusion process, and (ii) one can use the simpler volume-conserving theory to extract an effective fusion time with which we can then determine the characteristic fusion time.

For the even fusion case, we set $R_{10}=R_{20}=R_{0}, b=1$, and $h=1$, and Eq. 2.17 becomes,

$$
-\int_{0}^{\theta} \frac{g^{2}(\phi)}{f^{\prime}(\phi)} d \phi=2 \int_{0}^{\theta} d \phi \rho(\phi) \tan \phi=t / \tau_{s},
$$

where $\tau_{s}=\eta R_{0} / \gamma$. Setting $\rho(\phi) \approx 1$, results in the analytical approximation [9],

$$
\sin ^{2}(\theta) \approx 1-e^{-t / \tau_{s}}
$$

The theoretical models are tested below on simulations and experiments of fusing spherical aggregates, for both the identical and nonidentical cases, to validate the models. This is done by showing how the continuum theory can act as an intermediary to predict from simulation how nonidentical aggregates fuse in experiment. The first step in making predictions is to determine the CPD time unit in SI units, a process we call "calibration", by comparing the characteristic fusion times from identical aggregate fusion in simulation and experiment. 


\subsubsection{Calibration}

The calibration process consists of determining the CPD time unit, $T$, in terms of SI (experimental) units. The two equilibrated even (uneven) spherical aggregates were placed at a distance of one CP diameter apart and fused over 35 (50) million timesteps, $\Delta t=10^{-4} \mathrm{~T}$. The simulation required $\sim 8 \times 10^{3} \mathrm{~T} /$ day $\left(\sim 2.5 \times 10^{3} \mathrm{~T} /\right.$ day $)$ on 32 (24) processors for the even (uneven) system. For easy reference, the fusion simulations and experiments have been named according to Table. 2.2.

Table 2.2: Nomenclature

\begin{tabular}{ccc}
\hline \hline Name & Type & Initial Radii \\
\hline S1 & Simulation & \multirow{2}{*}{ Even } \\
E1 & Experiment & \\
\hline S2 & Simulation & \multirow{2}{*}{ Uneven } \\
E2 & Experiment & \\
\hline
\end{tabular}

The spherical multi-cellular aggregates used in the even spherical fusion simulation consisted of 500 cells each and had initial radii $R_{0}=10.2$. To determine the CPD time unit in SI units we first fit the fusing aggregates with the contour shown in Fig. 2.3, for the even case where $R_{1}=R_{2}=R$ and $\theta_{1}=\theta_{2}=\theta$ in Mathematica 9 [22]. This analysis generated a time series of contact angles, which were fit to the theoretical model, $q_{0 s}$, to determine the $\mathrm{S} 1$ characteristic fusion time, $\tau_{S 1}$.

The volume conserving theory fit well both the time series of the fusion angles and the radii values, as shown in Fig. 2.4. Snapshots were rendered, in VMD [23], with different colored cells for each aggregate to show that mixing does not occur during the simulation. For the spherical fusion process, $\tau_{S 1}=640$ and $t_{0}=-190$. Here, $t_{0}$ is the initial time when the fusing aggregates touch at a single point. The data is plotted as a function of $\Delta t / \tau$, with $\Delta t=t-t_{0}$. The negative initial time means that 
(a)

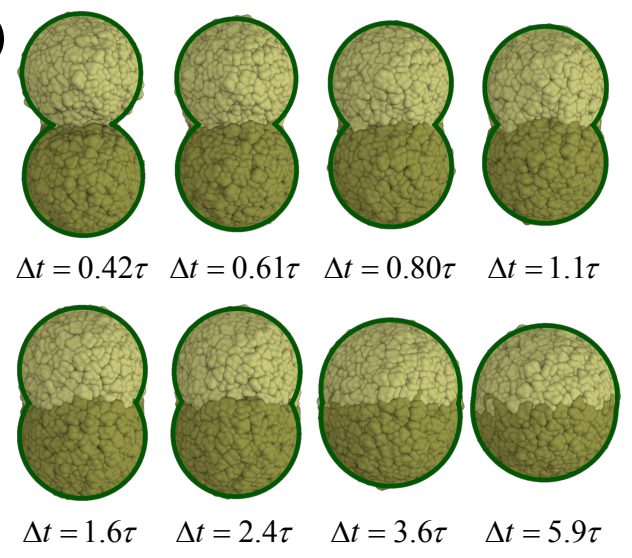

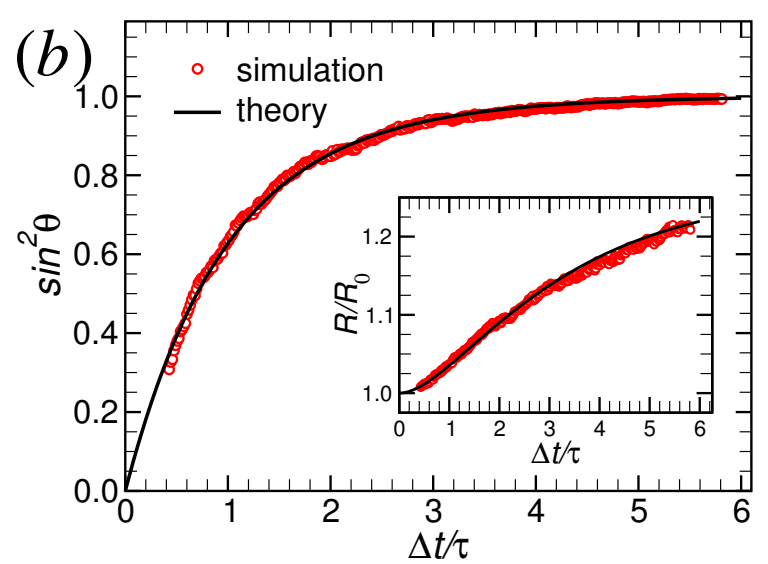

Figure 2.4: (a) Select snapshots from CPD simulation S1 of the fusion of two even (500 cell) spherical aggregates are displayed, with the superimposed fit. (b) The main graph shows the time dependence of $\sin ^{2}(\theta)$ during the simulation, along with the theoretical curve. The inset graph demonstrates how $R / R_{0}$ changes in the simulation compared against the theoretical $\rho_{0 s}(\theta)$, which assumes volume is conserved. The fusion time and the time corresponding to the initial state, attained from fitting the simulation data to the theoretical curve, were respectively $\tau_{S 1}=640$ and $t_{0}=-190$.

the aggregates had started to fuse before the first snapshot was taken.

Similar fusion experiments of identical spherical cellular aggregates, composed of human skin fibroblast (HSF) cells, were reported in [10]. The aggregates were prepared using the "egg holder aggregate maker" (EHAM) method and had initial radii $R_{0}=274 \mu \mathrm{m}$. The results are shown in Fig. 2.5.

The volume change parameter values, $a_{0}=0.73$ and $\lambda_{a}^{-1}=50$ hours, were determined from the volume loss data prior to fitting the contact angle values to determine the $\mathrm{S} 2$ characteristic fusion time. Significant volume loss is evident in the snapshots during the experiment, $V(\infty) / V_{0}=a_{0}^{3} \approx 0.4$, but this has little effect on the time evolution of the contact angles.

The volume conserving and volume change theory both fit the experimental fusion angle values well but only the volume change theory accurately models the time 

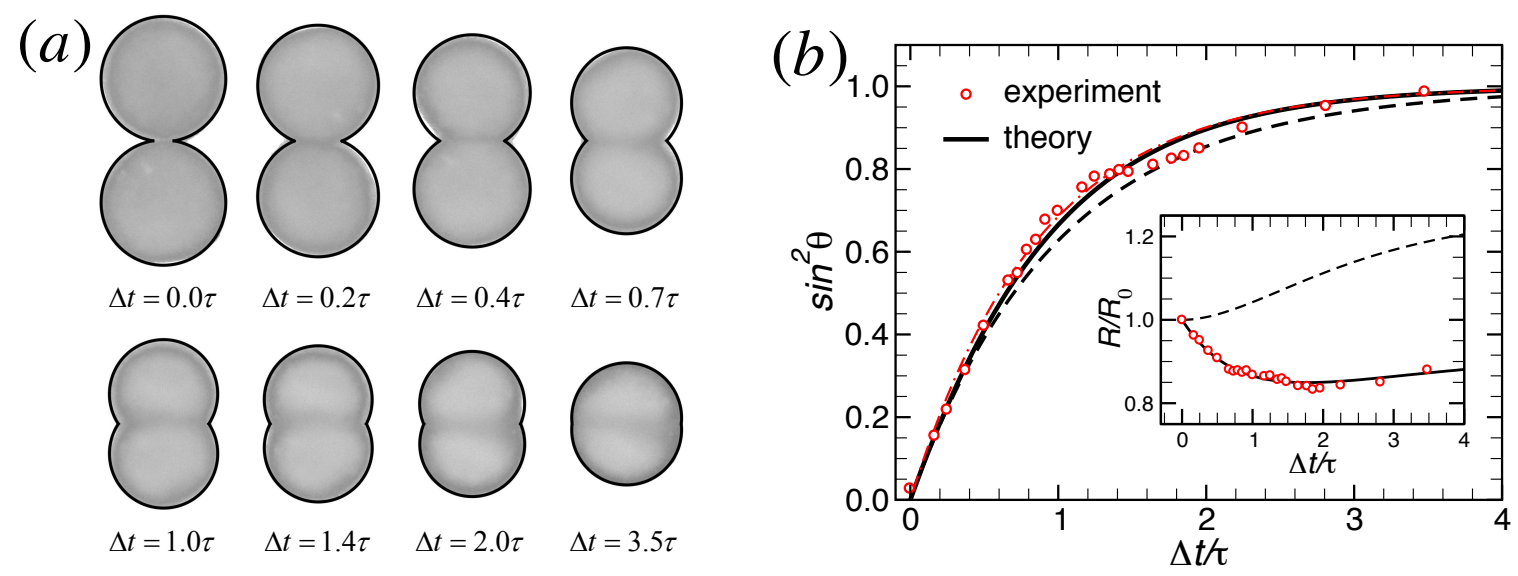

Figure 2.5: (a) Select snapshots from a fusion experiment E1 of two even $\left(R_{0}=\right.$ $274 \mu \mathrm{m})$ HSF spherical aggregates are displayed, with the superimposed fit. (b) The main graph shows the time dependence of $\sin ^{2}(\theta)$, along with the theoretical curves assuming volume conservation (dashed, $q_{0 s}$ ) and volume change (solid, $q_{s}$ ). The inset graph demonstrates how $R / R_{0}$ changes in the experiment compared against the volume conserving (dashed, $\rho_{0 s}$ ) and volume change (solid, $\rho_{s}$ ) theory. The parameter values from fitting were $\tau_{E 1}=48.0 \mathrm{~h}, t_{0}=9.3 \mathrm{~h}, a_{0}=0.73$, and $\lambda_{a}^{-1}=50 \mathrm{~h}$.

dependence in the radii values. The small effect on the fusion process is also evident in that the effective fusion time, obtained from fitting the experimental data to $q_{0 s}$, was $\tau_{E 1}^{*}=c_{a} \tau_{E 1}=41.8$ hours, similar to the characteristic fusion time, $\tau_{E 1}=48.0$ hours. Either fitting method is appropriate as long as the $c_{a}$ correction is made to $\tau_{s}^{*}$ when volume conserving theory is applied and $a(t)$ is used to scale the radii values.

If we use the volume conserving theory to fit both S1 and E1, then $q_{0 s}\left(\Delta t / \tau_{S 1} T_{1}\right)=$ $q_{0 s}\left(\Delta t / c_{a} \tau_{E 1}\right)$ where we now include the unit conversion parameter, $T_{1}$,

$$
T_{1}=c_{a} \tau_{E 1} / \tau_{S 1}=3.9 \mathrm{~min} .
$$

With the CPD time unit calibrated in SI units we can run a new simulation, i.e., uneven fusing spheres, and convert the CPD time into experimentally measured time, 
thus predicting the duration of the corresponding fusion experiment. This method was used in the past to make experimental predictions from simulation, but below a simpler method has been applied where $T_{1}$ can be found directly by fitting the E1 contact angle data, $\theta_{E 1}(\Delta t)$, directly with the simulation data, $\theta_{S 1}\left(\Delta t / T_{1}\right)$, without ever determining the characteristic fusion times or making use of the intermediate continuum theory.

\subsubsection{Validation}

To validate the uneven spherical fusion theoretical models, we have also performed CPD simulations and experiments involving the fusion of two nonidentical spherical aggregates. In the simulation, S2, the larger and smaller aggregates had an initial radius of $R_{10}=15.9$ and $R_{20}=9.4$, respectively, for an initial ratio, $b=R_{10} / R_{20}=$ 1.7. The volume-conserving theory fit extremely well the $\mathrm{S} 2$ simulation data, Fig 2.6,
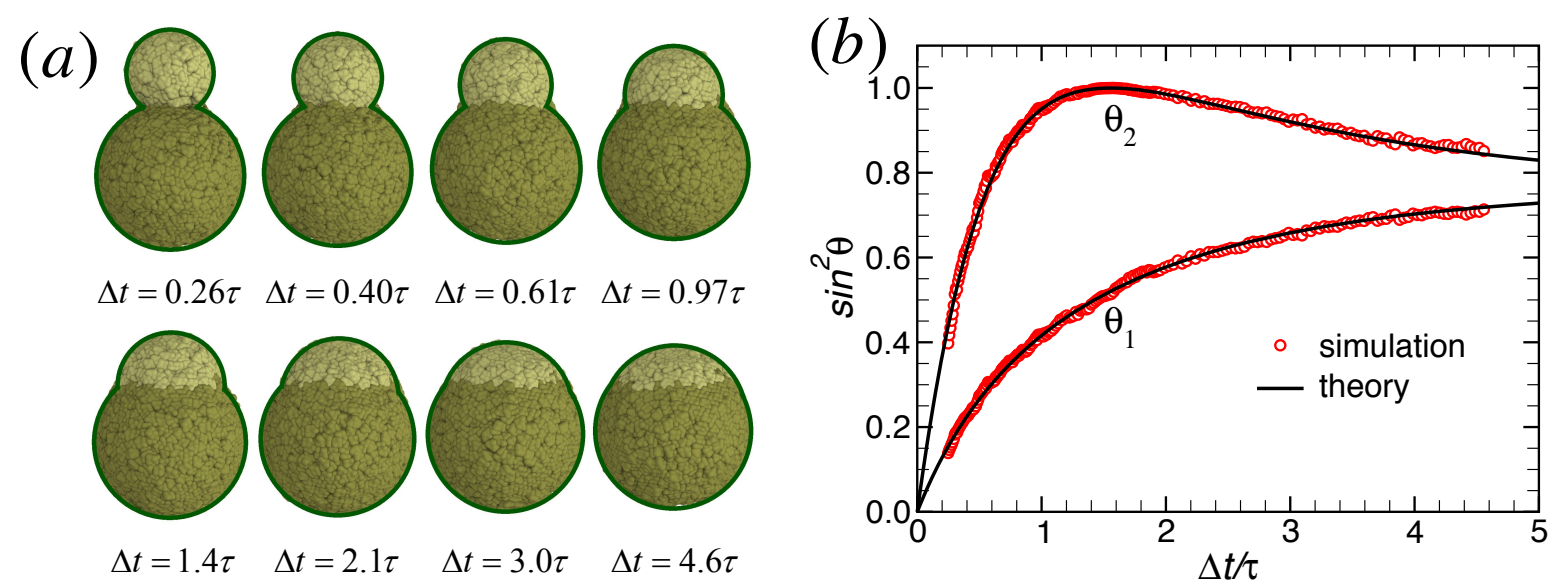

Figure 2.6: (a) Select snapshots from CPD simulation S2 of the fusion of two uneven spherical aggregates $(b=1.7)$ are displayed, with the superimposed fit. (b) The main graph shows the time dependence of $\sin ^{2}\left(\theta_{1,2}\right)$ during the simulation, along with the theoretical curves. The characteristic fusion time $\tau_{S 2}$ and $t_{0}$ were 1120 and -190 , respectively. 
and provided a characteristic fusion time $\tau_{S 2}=1120$, along with $t_{0}=-190$.

For the uneven fusion experiment, E2, also using HSF cells prepared with EHAM, the volume-conserving theoretical model again fit well the contact angle values, Fig. 2.7, but failed to follow the radii values due to volume loss (not shown). The initial sizes of the aggregates were $R_{10}=302 \mu \mathrm{m}$ and $R_{20}=178 \mu \mathrm{m}$, resulting in $b=1.7$ which was identical to S2. The characteristic fusion time was $\tau_{E 2}=48.9$, and $t_{0}=-3.0$ hours.
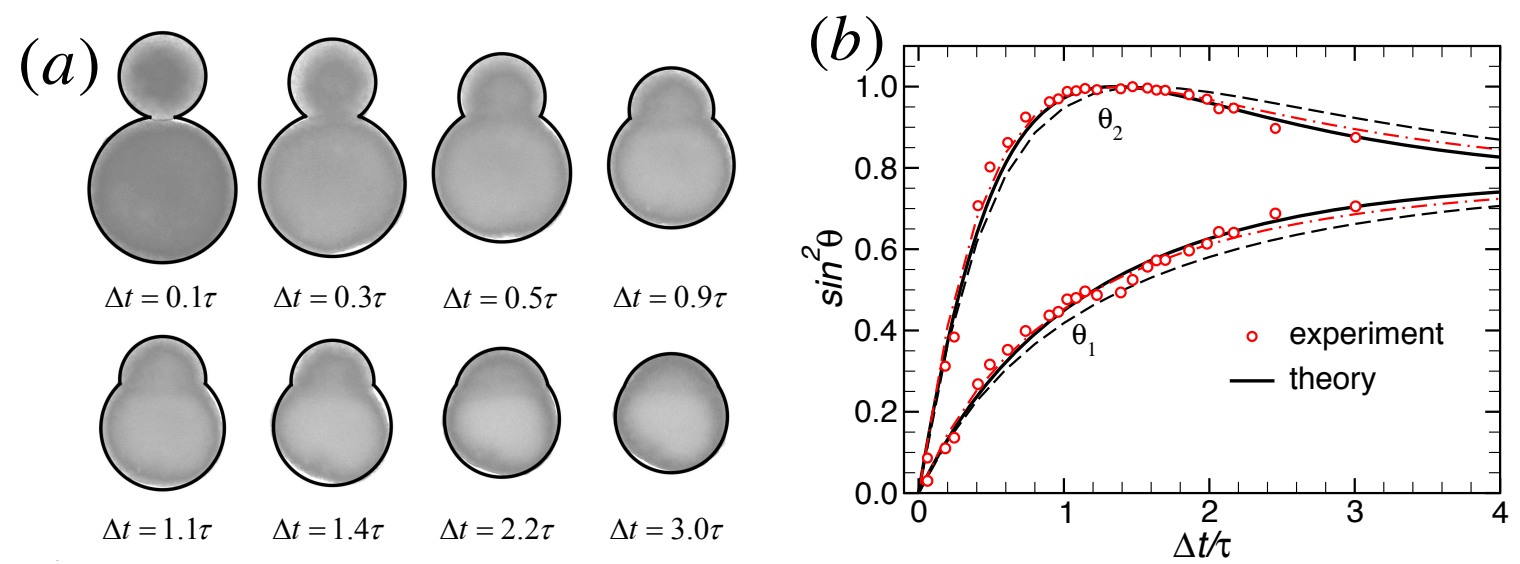

Figure 2.7: (a) Select snapshots from fusion experiment E2 of two uneven HSF spherical aggregates $(b=1.7)$ are displayed, with the superimposed fit. (b) The graph shows the time dependence of $\sin ^{2}\left(\theta_{1,2}\right)$, along with the theoretical curve which assumes volume change (solid, $q_{s}$ ). Two volume conserving curves are also shown: $q_{0 s}\left(\Delta t / \tau_{E 2}\right)$ (black dashed) and $q_{0 s}\left(\Delta t / c_{a} \tau_{E 2}\right)$ (red, dashed-dotted). The parameter values from fitting were $\tau_{E 2}=48.9 \mathrm{~h}, t_{0}=-3.0 \mathrm{~h}, a_{0}=0.67$, and $\lambda_{a}^{-1}=52.6 \mathrm{~h}$.

In Fig. 2.7, the volume change $q_{s}\left(\Delta t / \tau_{E 2}\right)$ is shown along with two volume conserving curves, i.e., $q_{0 s}\left(\Delta t / \tau_{E 2}\right.$ (black dashed) and $q_{0 s}\left(\Delta t / c_{a} \tau_{E 2}\right.$ (red, dashed-dotted), respectively. When the volume of the system decreases during the fusion process, there is a noticeable difference in the time dependence of the contact angles compared to the situation when the volume does not change. However, the volume conserving 
model can predict the contact angles if the effective fusion time (i.e., $c_{a} \tau_{E 2}$ ) is used instead of $\tau_{E 2}$. A list of the values of the fitting parameters is provided in Table 2.3.

Table 2.3: Fitting Parameter Values

\begin{tabular}{ccccccc}
\hline \hline & $\tau$ & $t_{0}$ & $a_{0}$ & $\lambda_{a}^{-1}$ & $R_{10}$ & $\mathrm{~b}$ \\
\hline S1 & 640 & -190 & 1 & & 10.2 & 1 \\
$\mathrm{E} 1$ & 48.0 & 9.3 & 0.73 & 50 & 274 & 1 \\
\hline S2 & 1120 & -190 & 1 & & 15.9 & 1.7 \\
$\mathrm{E} 2$ & 48.9 & -3.03 & 0.67 & 52.6 & 302 & 1.7 \\
\hline
\end{tabular}

The time dependence of the volume for all four simulations and experiments is shown in Fig. 2.8 where no volume change occurs in simulation (S1, S2) and both experiments follow the volume loss model, Eq. 2.19, with parameter values for both near $a_{0}=0.7$ and $\lambda_{a}^{-1}=50$. The fact that the values of the volume loss parameters are nearly identical in both experiments suggests that they only depend on the cell type and preparation method used to create the aggregates. Note that we can also

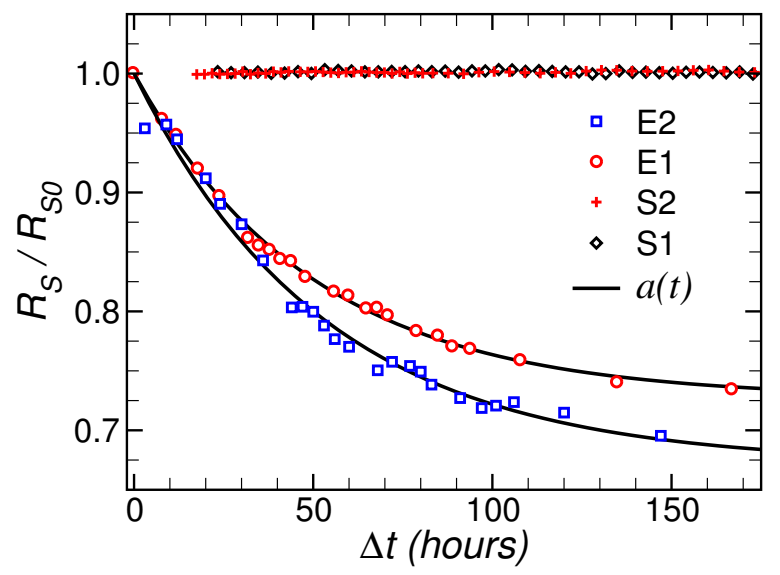

Figure 2.8: The time dependence of the volume is shown for each simulation and experiment. In simulation (S1, S2) the volume does not change, $R_{S} / R_{S 0}=1$. In experiment, the parameter values were $a_{0}=0.73(0.67)$ and $\lambda_{a}^{-1}=50(52.6)$ hours for $\mathrm{E} 1(\mathrm{E} 2)$. Note that $R_{S} / R_{S 0}=[V(t) / V(0)]^{1 / 3}=a(t)$.

determine the values of the volume change parameters from a single aggregate whose 
volume decreases in time.

\subsubsection{Prediction}

As already mentioned, the CPD time unit, $T_{1}$, in SI units can also be determined directly, by using the $\theta_{S 1}$ data to fit the time dependent contact angles from $E 1$. The result is shown in Fig. 2.9(b), where the same value $T_{1}=3.9$ min was obtained as in Eq. 2.29. The radii and contact angle values from simulation $\mathrm{S} 1$ have been used to

(a)

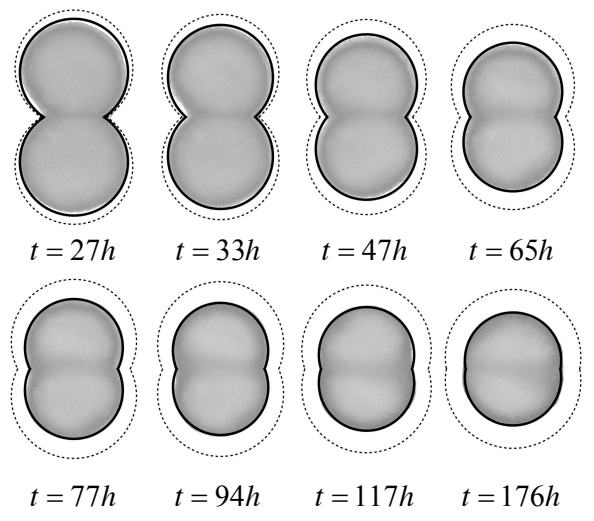

(b)

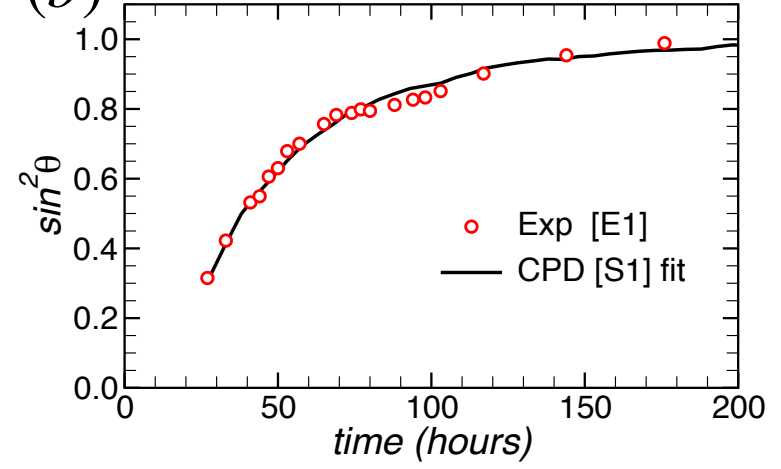

Figure 2.9: (a) Snapshots from the HSF even fusion experiment, E1, with the solid (dashed) contour from S1 where the radii values include (do not include) the radii scaling from volume loss taken in to account. (b) The simulation data, $\theta_{S 1}$, has been directly fit on to experiment E1 to determine the time conversion value. Here $T_{1}=3.9$ min and $\Delta t=-21.6$ hours.

produce the solid (dotted) contour, Fig. 2.9, with (without) scaling the radii values with $a(t)$ from Eq. 2.20.

Following a similar process as in Eq. 2.29, we can determine the time unit conversion factor between S2 and E2 for the uneven systems

$$
T_{2}=c_{a} \tau_{E 2} / \tau_{S 2}
$$


Solving for $c_{a}$ in Eq. 2.29 and substituting the result into Eq. 2.30, one obtains

$$
T_{2} \equiv T_{2, \tau} \approx T_{1} \frac{\tau_{E 2}}{\tau_{E 1}} \frac{\tau_{S 1}}{\tau_{S 2}}
$$

where, assuming that $\eta / \gamma$ is constant, one can make a substitution for $\tau$ from Eq. 2.18 that leads to

$$
T_{2} \equiv T_{2, R} \approx T_{1} \frac{R_{10, E 2}}{R_{0, E 1}} \frac{R_{0, S 1}}{R_{10, S 2}} .
$$

Note that the value of $T_{2, R}$ can be determined without any contribution from the theoretical model. With the known data we can determine $T_{2, \tau}=2.3 \mathrm{~min}$ and $T_{2, R}=2.7 \mathrm{~min}$. The small difference between the two values is likely due to the fact that the value of $\eta / \gamma$ may slightly differ from one experiment (simulation) to another.

With $T_{2, R}$ we can use $\mathrm{S} 2$ to predict the time evolution of the contour shape in experiment E2. The time dependence of $q_{S 2}\left(\Delta t / T_{2}\right)$, along with the corresponding experimental data, is shown in Fig. 2.10; the solid (dashed) contours take into account (neglect) the volume loss during fusion. While $T_{2, \tau}$ provides a better fit to the experimental contact angle data than $T_{2, R}$, there is no difference in the predicted contours drawn on the experimental snapshots.

This clearly demonstrates that volume conserving CPD simulations can predict the volume changing experimental fusion of uneven spheroidal aggregates without using the intermediate theoretical continuum model. Thus, the presented method is capable of predicting the shape evolution of arbitrary multicellular constructs following 3D bioprinting. 
(a)
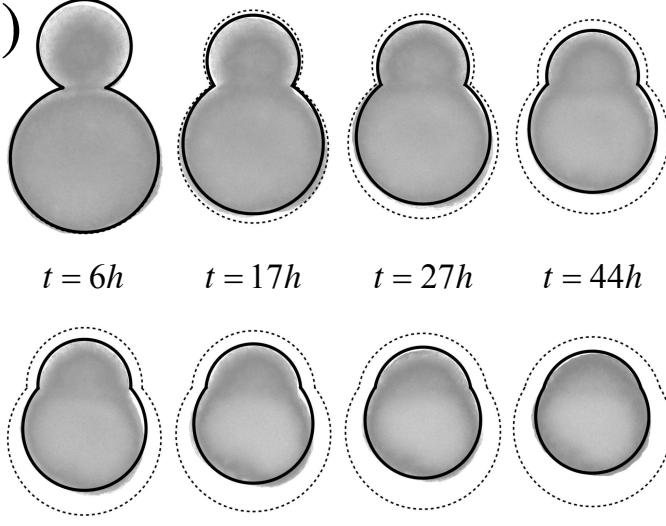

$t=57 h$

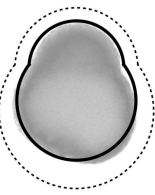

$t=77 h$ $t=27 h$

$t=44 h$

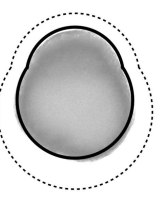

$t=98 h$

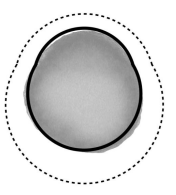

$t=144 h$

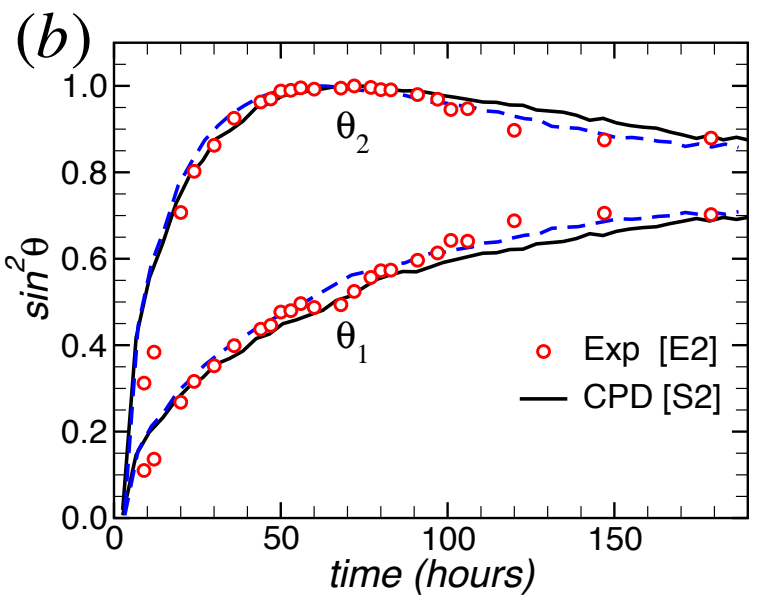

Figure 2.10: (a) Snapshots from E2 with contour predictions from S2 with (without) the volume loss taken into account for the solid (dotted) contours and (b) the S2 prediction curves plotted on the E2 experimental data using $T_{2, R}\left(T_{2, \tau}\right)$ for the solid (dashed) curves.

\subsection{Fusion of Cylindrical Aggregates}

To test the CPD formalism on another bioink geometry typically used in experiments, fusion simulations and experiments were also carried out for cylindrical aggregates composed of the same HSF cells as the spherical aggregates considered above. However, these fusion experiments used a different technique to prepare the bioink, named the "sausage method" (SM). Details of SM can be found in Ref. [11]. Even though the same cell type was used, calibration had to be repeated because the new preparation technique resulted in different values for the physical constants, $\eta$ and $\gamma$, and possibly other fitting parameters.

Another complication during the fusion of cylindrical aggregates is that the sample is destroyed each time a measurement is made because the aggregates need to be cut to analyze their cross sections to determine $R$ and $\theta$. This means that the cylinder fusion data at each time step represents a completely independent system. 
It is also possible that independent pairs of cylinders are at different stages of the fusion process at the initial time $t_{0}$ and even within a single fusing pair the fusion process may not be identical along the length of the cylinders. To account for this, at each time step multiple systems and multiple locations along the length of a single fusing pair were measured. For each time step, the results were graphed as the mean, with error bars given by the standard deviation. For spherical aggregates, where the fusion process can be monitored in its entirety, a similar plotting procedure was used by averaging the obtained fusion data for three independent systems.

\subsubsection{Theory}

During the fusion process of two even cylindrical aggregates, if the volume and length of the aggregates do not change, then the radius $R$ is entirely determined by the contact angle $\theta$, once the initial size, $R_{c 0}=R_{c}\left(t_{0}\right)$, of the system has been measured,

$$
\rho_{0 c}(\theta) \equiv \frac{R_{c}}{R_{c 0}}=\left(1-\frac{\theta}{\pi}+\frac{\sin 2 \theta}{2 \pi}\right)^{-1 / 2}
$$

We again solve for the time dependence of the contact angle using energy conservation as in Eq. 2.5,

$$
d \sigma_{c} / d t=-2 \tau_{c} u^{2}
$$

where $u$ is the strain rate tensor, $u=-\rho_{c}^{-1} d\left(\rho_{c} \cos \theta\right) / d t \approx g_{c}(\theta) \dot{\theta}$, and

$$
\sigma_{c}=(1-\theta / \pi) \rho_{c} z
$$


where $z=L / L_{0}$ is the normalized length of the cylinder, $\rho_{c}=R / R_{0}$ is the normalized radius, and the characteristic fusion time, $\tau_{c}$, for cylinders is

$$
\tau_{c}=(\eta / \gamma) R_{c 0}
$$

Assuming volume conservation we can determine the renormalized radius, $\rho_{c}=$ $\rho_{0 c} z^{-1 / 2}$. Inserting this into Eq. 2.35, the normalized surface area becomes $\sigma_{c}=$ $f_{c}(\theta) z^{1 / 2}(t)$, where $f_{c}(\theta)=(1-\theta / \pi) \rho_{0 c}(\theta)$. Inserting these definitions into Eq. 2.34,

$$
\dot{\theta}=-\alpha / 2\left[1+\left(1-\beta / \alpha^{2}\right)^{1 / 2}\right]
$$

where

$$
\begin{aligned}
& \alpha=f_{c}^{\prime}(\theta) / 2 \tau_{c} g_{c}^{2}(\theta) \zeta(t), \\
& \beta=f_{c}(\theta) h(t) / \tau_{c} g_{c}^{2}(\theta) \zeta(t),
\end{aligned}
$$

with $f_{c}^{\prime}(\theta) \equiv d f_{c}(\theta) / d \theta, h(t)=(1 / z) \dot{z}$, and $\zeta \equiv \rho_{c} / \rho_{0 c}=z^{-1 / 2}$.

When the length of the cylinder does not change, i.e., $z=1$, then $\beta=0$ and Eq. 2.37 becomes $\dot{\theta}=-\tau_{c}^{-1} f_{c}^{\prime} / 2 g_{c}^{2}$. By integrating this, one obtains the implicit expression of the time dependent contact angle $\theta(t)$,

$$
\int_{0}^{\theta} \frac{\left[(\pi-\phi)^{2} \sin ^{2} \phi \tan \phi /[(\pi-\phi) \cos \phi+\sin \phi]\right]}{(1-\phi / \pi+\sin 2 \phi / 2 \pi)^{1 / 2}} d \phi=t / 2 \tau_{c}
$$

For cylinders the results are reported as

$$
r / R=\sin \theta \equiv q_{0 c}\left(t / \tau_{c}\right)
$$


In cylinder fusion experiments the volume decrease can be modeled similarly to spheres, i.e., by using $a(t)$ (see Eq. 2.19). The length, $L$, of the cylindrical aggregates decreases according to

$$
z(t) \equiv L / L_{0}=b_{0}+\left(1-b_{0}\right) \exp \left(-\lambda_{b} t\right)
$$

where $b_{0}=L / L_{0}$ for $t \gg \lambda_{b}^{-1}$, with $\lambda_{b}^{-1}$ being the characteristic length relaxation time. The volume of our system is give by

$$
V(t)=V_{0} a^{3}(t)=V_{0}\left(\rho_{c} / \rho_{0 c}\right)^{2} z(t),
$$

which redefines $\zeta=\rho_{c} / \rho_{0 c}=z^{-1 / 2}(t) a^{3 / 2}(t)$. Substituting $\zeta$ into Eq. 2.37 leads to [11]

$$
\sin \theta \equiv q_{c}\left(t / \tau_{c} ; b_{0}, \lambda_{b} \tau_{c} ; a_{0}, \lambda_{a} \tau_{c}\right) .
$$

Similarly to the spherical fusion case where $q_{0 s}\left(t / \tau_{s}^{*}\right) \approx q_{s}\left(t / \tau_{s} ; a_{0}, \lambda_{a} \tau_{s}\right)$, for cylinders

$$
q_{c}\left(t / \tau_{c} ; b_{0}, \lambda_{b} \tau_{c} ; a_{0}, \lambda_{a} \tau_{c}\right) \approx q_{0 c}\left(t / \tau_{c}^{*}\right) .
$$

However, unlike in the case of fusion of spherical aggregates, where $\tau_{s}^{*}=c_{a} \tau_{s}$, for cylindrical aggregates there is no simple approximation to determine the relationship between $\tau_{c}^{*}$ and $\tau_{c}$. For cylinders, the values for $b_{0}, \lambda_{b}, a_{0}$, and $\lambda_{a}$ must be predetermined and inserted into Eq. 2.45 to determine the relationship between $\tau_{c}^{*}$ and $\tau_{c}$. 


\subsubsection{Calibration}

Again, for simple reference, all four of the even fusion simulations and experiments have been named according to Table 2.4. A similar procedure is followed for calibration, using an even spherical fusion simulation and experiment to determine $T_{1}$. The simulation S1, for even fusing spheres, Fig. 2.4, does not need to be repeated. As a reminder, the characteristic fusion time for $\mathrm{S} 1$ was $\tau_{S 1}=640$.

Table 2.4: Nomenclature

\begin{tabular}{ccc}
\hline \hline Name & Type & Initial Radii \\
\hline S1 & Simulation & \multirow{2}{*}{ Spheres } \\
E1 & Experiment & \\
\hline S2 & Simulation & \multirow{2}{*}{ Cylinders } \\
E2 & Experiment & \\
\hline
\end{tabular}

The even fusion experiment E1, using SM, had initial radii $R_{0}=220 \mu \mathrm{m}$. The volume change parameter values were $a_{0}=0.76$ and $\lambda_{a}^{-1}=50$ hours, a characteristic volume relaxation time identical to what was found for E1 with EHAM aggregates. The characteristic fusion time and initial time were determined to be $\tau_{E 1}=25$ and $t_{0}=6.9 \mathrm{~h}$, solid curve in Fig. 2.11. Also shown is $q_{0 s}$ with the reduced fusion time, $\tau_{E 1}^{*} \approx \tau_{S 1}\left(1+a_{0}\right) / 2=22 \mathrm{~h}$. While both $q_{s}$ and $q_{0 s}$ can accurately describe the time dependence of the contact angles, only the volume change theory, $\rho_{s}$, can correctly model the time evolution of the radii values.

\subsubsection{Validation}

For the even cylindrical fusion simulation, two equilibrated cylinders with 1535 cells each, the same initial radii, $R_{0}=10.2$, as the spherical aggregates, and a length of roughly $4 R_{0}$, were placed a distance of one $\mathrm{CP}$ diameter apart. Periodic boundary 

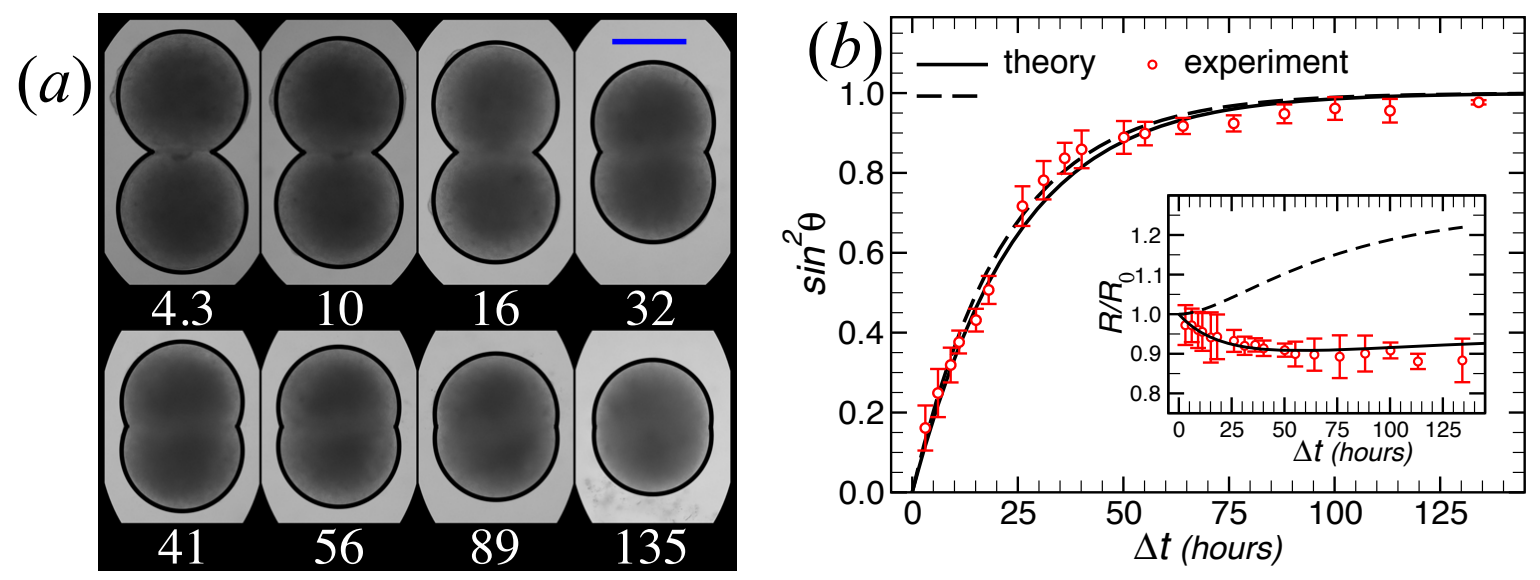

Figure 2.11: (a) Pictures, with time in hours, of two fusing HSF spherical aggregates in experiment E1 with the superimposed fit. The scale bar length is $250 \mu \mathrm{m}$. (b) The experimental data (red circles) was averaged over three experiments and the standard deviation shown as the error bar. The graph shows the measured contact angles, along with $q_{s}$ (solid) and $q_{0 s}$ (dashed). The inset graph demonstrates that the volume conserving curve, $\rho_{0 s}$ (dashed), fails to properly fit the measured radii while $\rho_{s}$ (solid) does quite well. From fitting, $\tau_{E 1}=25 h$ and $t_{0}=6.9 h$ (and the effective fusion time, $\tau_{s}^{*}=22 h$ ) were determined.

conditions were used in the axial direction of the cylinders, rendering them quasiinfinite. The same parameter values were used as in the previous CPD simulations and the performance was $\sim 3 \cdot 10^{3} \mathrm{~T}$ /day on 24 processors. The results are shown in Fig. 2.12 where the characteristic fusion time and initial time were $\tau_{S 2}=540$ and $t_{0}=-30$.

For the even cylinder fusion experiment E2, each data point shown in Fig. 2.13 represents an average of multiple locations along the fusing aggregates and multiple pairs of aggregates, where 6-10 measurements were taken for each point. Before determining the characteristic fusion time, the following parameters were determined from the length contraction data: $b_{0}=0.4$ and $\lambda_{b}^{-1}=20.8 \mathrm{~h}$. Also, the volume change parameter values from E1 were used for E2: $a_{0}=0.76$ and $\lambda_{a}^{-1}=50 \mathrm{~h}$. The 
(a)
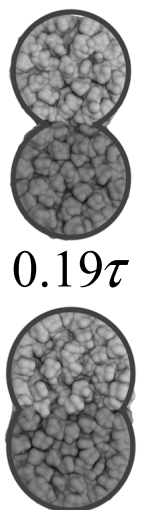

$2.0 \tau$
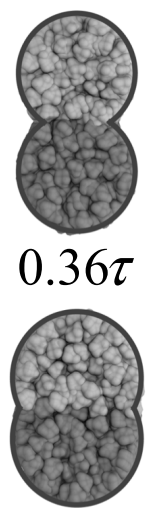

$3.0 \tau$
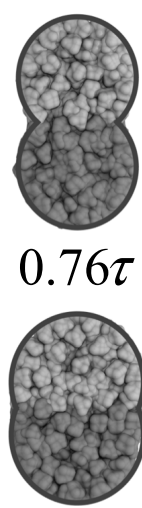

$4.0 \tau$
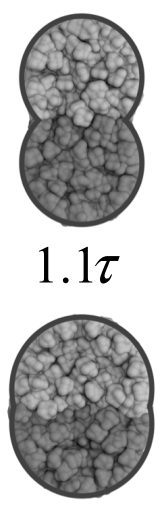

$5.9 \tau$

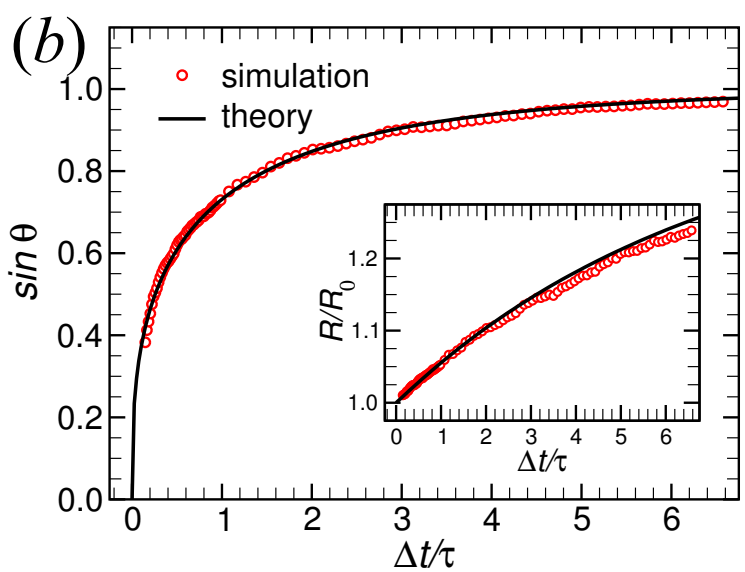

Figure 2.12: (a) Transaxial snapshots from CPD simulation S2 of the fusion of two even (1535 cell) cylindrical aggregates are displayed, with the superimposed fit. (b) The main graph shows the time dependence of $\sin (\theta)$ during the identical cylinder fusion simulation, along with the theoretical curve. The inset graph demonstrates how $R_{c} / R_{0}$ changes in the simulation compared against the theoretical $\rho_{0 c}(\theta)$, which assumes volume is conserved. The fusion time $\tau_{S 2}=540$, and the time corresponding to the initial state $t_{0}=-30$.

cylindrical characteristic fusion time and initial time were $\tau_{E 2}=19 \mathrm{~h}$ and $t_{0}=5.2 \mathrm{~h}$, respectively.

For spheres, the measured contour radii values decrease as the volume decreases but for cylinders the length contraction adds material to the cross section at a quicker rate and, therefore, the measured radii values increase, as seen in $\mathrm{E} 2$, where $R_{c} / R_{0}$ and $\rho_{c}$ are greater than predicted by the volume conserving theory, $\rho_{o c}$. Similarly to previous experiments, while the volume conserving theory can describe the time evolution of the contact angles, it fails to accurately model the change in the radii values.

As shown in Eq. 2.45, if we equate $q_{0 c} \approx q_{c}$, with the known values for $a_{0}, b_{0}$, $\lambda_{a}$, and $\lambda_{b}$, we can determine the effective fusion time, $\tau_{E 2}^{*}=10 \mathrm{~h}$. The fact that $\tau_{E 2}$ is about twice $\tau_{E 2}^{*}$ implies that the fusion process for cylinders occurs twice as 

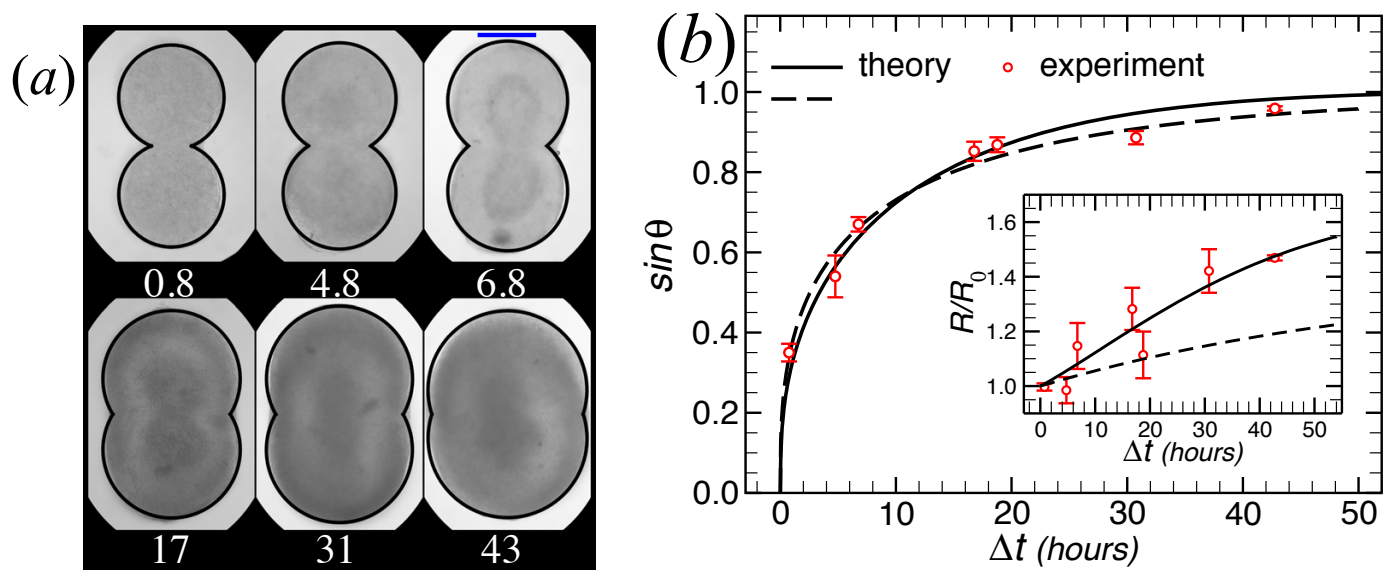

Figure 2.13: (a) Pictures, with time in hours, of the transaxial cross section of two fusing HSF cylindrical aggregates in experiment with the superimposed fit. The scale bar length is $250 \mu \mathrm{m}$. (b) The experimental data (red circles) was averaged over three experiments and the standard deviation shown as the error bar. The graph shows the measured contact angles, along with $q_{c}$ (solid) and $q_{0 c}$ (dashed). The inset graph demonstrates that the volume conserving curve, $\rho_{0 c}$ (dashed), fails to properly fit the measured radii while $\rho_{c}$ (solid) performs better. From fitting, $\tau_{E 2}=19 \mathrm{~h}$ and $t_{0}=5.2 \mathrm{~h}$ (and the effective fusion time, $\tau_{E 2}^{*}=10 \mathrm{~h}$ ) were determined.

fast for the volume and length changing case versus the conserved case. Also, since $R_{s 0}=220 \mu \mathrm{m} \approx R_{c 0}=225 \mu \mathrm{m}$, we should expect $\tau_{s} \approx \tau_{c}$ but interestingly in both experiment and simulation $\tau_{c}$ is $15-20 \%$ smaller than $\tau_{s}$. There may be a geometric dependence to $\tau$ that is not captured by our theoretical models.

A summary of all the simulation and experimental results for cylindrical aggregates is shown in Table 2.5

Table 2.5: Fitting Parameter Values (Values with * were enforced)

\begin{tabular}{cccccccc}
\hline \hline & $\tau$ & $t_{0}$ & $R_{0}$ & $a_{0}$ & $\lambda_{a}^{-1}$ & $b_{0}$ & $\lambda_{b}^{-1}$ \\
\hline S1 & 640 & -190 & 10.2 & 1 & & & \\
E1 & 25 & 6.9 & 220 & 0.76 & 50 & & \\
\hline S2 & 540 & -30 & 10.2 & 1 & & 1 & \\
E2 & 19 & 5.2 & 225 & $0.76^{*}$ & $50^{*}$ & 0.4 & 20.8 \\
\hline
\end{tabular}


The volume loss and length contraction data is shown in Fig. 2.14. The values of the volume change parameters for spheres do reasonably well to model the volume loss for cylinders.

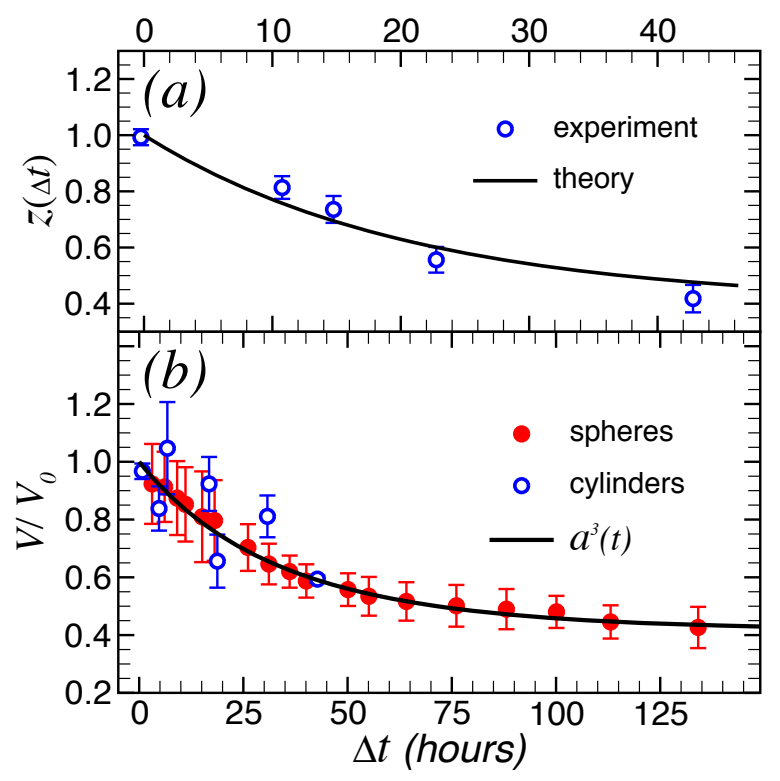

Figure 2.14: (a) The time dependence of the relative cylinder length, $L / L_{0}$, is displayed along with theoretical model, $z(t)$, that models length contraction where $b_{0}=0.4$ and $\lambda_{b}^{-1}=20.8 \mathrm{~h}$. (b) The volume decrease for both spheres (E1) and cylinders (E2) during fusion fits well the theoretical model $a(t)$ with $a_{0}=0.76$ and $\lambda_{a}^{-1}=50 \mathrm{~h}$.

\subsubsection{Prediction}

The predictive power of CPD is demonstrated in Fig. 2.15. First, the sphere fusion simulation (S1) and experiment (E1) are used to calibrate the CPD time unit in SI units. Then, the cylinder fusion simulation (S2) results are plotted against the cylinder fusion experimental (E2) data. The following steps were followed to obtain these results. 
1. Determine the time unit conversion factor $T_{1}$ for spherical fusion by fitting the $\mathrm{S} 1$ data with the E1 data [inset figure in Fig. 2.15(a)]: $T_{1}=0.035 \mathrm{~h}$.

2. Determine the effective fusion time in experiment E1: $\tau_{E 1}^{*}=\tau_{S 1} T_{1}=22.4 \mathrm{~h}$.

3. Calculate the characteristic fusion time for experiment E1: $\tau_{E 1}=\tau_{E 1}^{*}\left(1+a_{0}\right) / 2=$ $25.5 \mathrm{~h}$, with $a_{0}$ determined from the volume change data.

4. Calculate the characteristic fusion time for experiment E2: $\tau_{E 2}=\tau_{E 1}\left(R_{0, E 2} / R_{0, E 1}\right)=$ $26 \mathrm{~h}$. This assumes the material constant ratio $\eta / \gamma$ does not change.

5. Use $q_{c}\left(t / \tau_{E 2} ; a_{0}, \lambda_{a} \tau_{c} ; b_{0}, \lambda_{b} \tau_{c}\right) \approx q_{0 c}\left(t / \tau_{E 2}^{*}\right)$ to determine the effective fusion time in experiment $\mathrm{E} 2: \tau_{E 2}^{*}=11.6 \mathrm{~h}$.

6. Determine the time unit conversion factor $T_{2}$ for cylindrical fusion: $T_{2}=$ $\tau_{E 2}^{*} / \tau_{S 2}=0.022 \mathrm{~h}$.

Following these steps, we then plotted the time evolution of the contact angle for S2 and E2, Fig. 2.15(a), which shows very good agreement between the two. Finally, with the S2 radii values, we can predict the time evolution of the contours in E2, both with (solid) and without (dashed) taking into account the volume and length change, Fig. 2.15(b). When the volume decreases and the length contracts then the radii are scaled with $R_{E 2} / R_{0, E 2}=\rho_{S 2} \cdot a^{3 / 2} \cdot z^{-1 / 2}$.

\subsubsection{Application}

For a system with a more complicated geometry, Fig. 2.16, consisting of many spherical or cylindrical aggregates that fuse to create a tubular structure, we have similar 

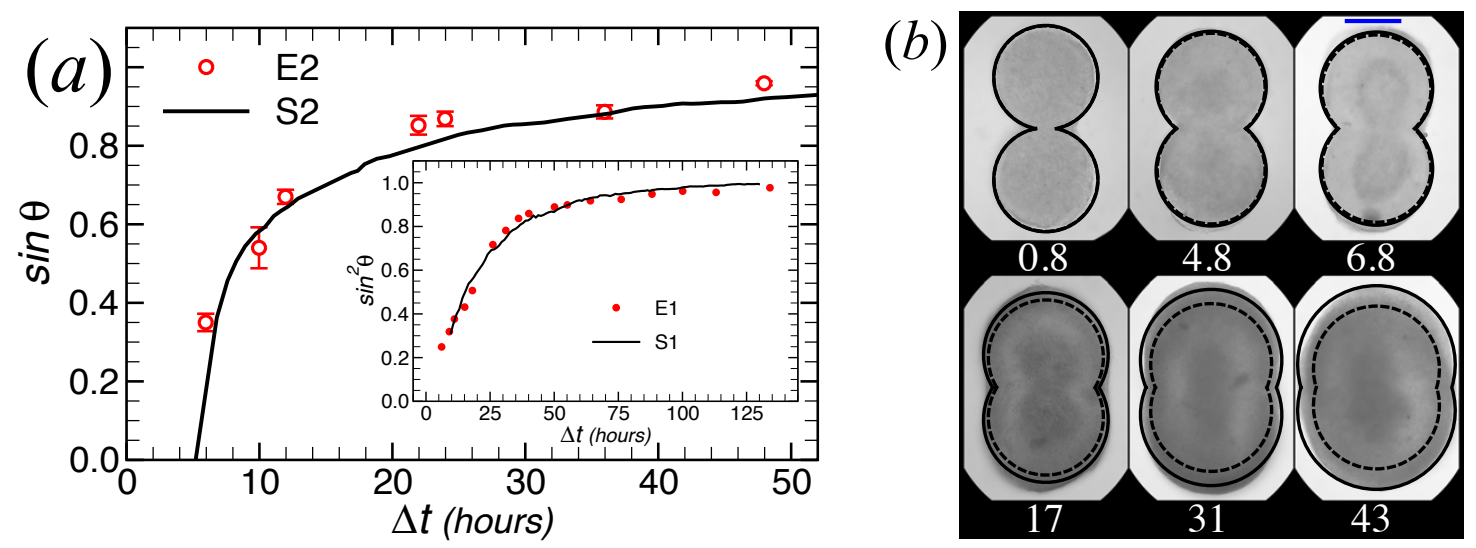

Figure 2.15: (a) The cylinder fusion (E2) experimental data (red circles) plotted with the CPD prediction from the cylinder fusion simulation (S2). The inset shows how the sphere fusion simulation data (S1) was used to fit the experimental data (E1) to determine the CPD time unit in SI units. (b) Pictures, with time in hours, of the transaxial cross section of two fusing HSF cylindrical aggregates in experiment with the CPD contour prediction shown, both with (solid) and without (dashed) volume and length change taken into account.

progression in the fusion in the simulations, since the volume and length do not change. To determine the progression in experiment we need to calculate the CPD time unit for each in SI units. For spheres, $T_{s}=T_{1}=0.035 \mathrm{~h}$ from step 1 above. For cylinders, $T_{c}=T_{2}=0.022 \mathrm{~h}$. Thus, $\Delta t=620$ in simulation corresponds to $\Delta t \cdot T_{s}=21.7 \mathrm{~h}$ and $\Delta t \cdot T_{c}=13.6 \mathrm{~h}$ in experiment, which means that cylinders would take about half as long to reach this state in experiment and therefore would be the preferred bioink to use in these applications.

\subsection{Conclusion}

The CPD formalism adds a theoretical foundation and quantitative modeling to an experimental technique, bioprinting, that has typically been based on trial and error. 


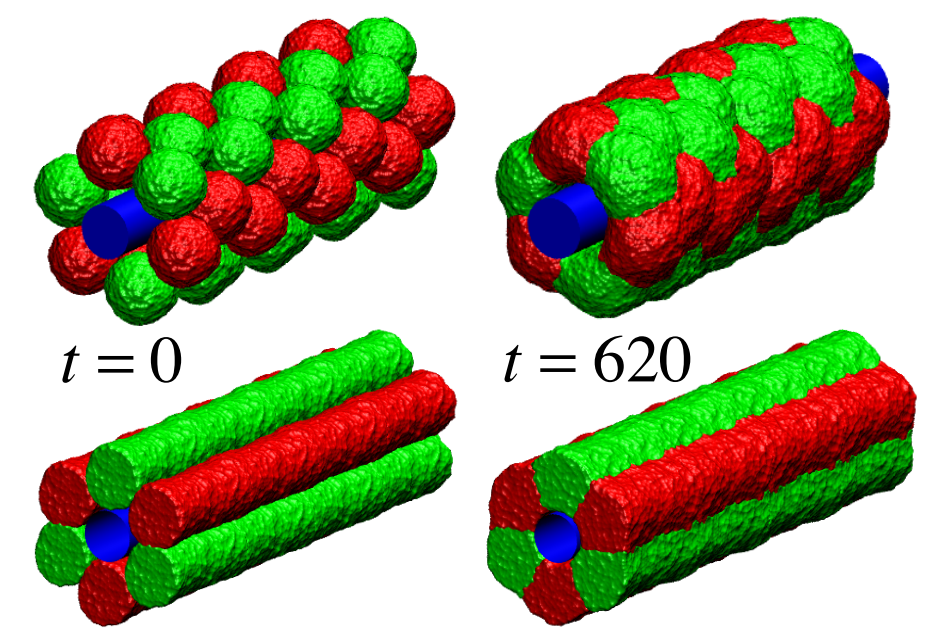

Figure 2.16: Snapshots taken at the beginning and near the end of the fusion of spheres (top) and cylinders (bottom) in a stacked geometry. The fusion process is at a similar stage of completion at $\Delta t=620$ in the simulation where volume and length are conserved. The calculated CPD time units for each are significantly different, $T_{s}=0.035 \mathrm{~h}$ and $T_{c}=0.022 \mathrm{~h}$, meaning the cylinder fusion in experiment will be much faster.

This collaborative effort was intended to help make predictions that improve the efficiency and reproducibility of bioprinting experiments. In this work, we have shown that (i) the CPD formalism can be extended to the fusion of uneven spherical aggregates, (ii) volume loss and length contraction of bioink during the post-printing fusion process can be well described by simple analytical expressions, (iii) using spherical bioink prepared using SM speeds up fusion, (iv) cylindrical bioink, due to volume loss and length contraction, fuses nearly twice as fast as spherical bioink and therefore is the preferable bioink to use when possible, and (v) the volume and length conserving CPD simulations can directly predict structure formation even when the volume and length do change in experiments and even in cases where the system has a more complex geometry that makes theoretical modeling undesirable. 


\section{Chapter 3}

\section{Structure and dynamics of lipid and water molecules in free-standing and supported hydrated phospholipid bilayer systems}

\subsection{Introduction}

All living cells are isolated from the environment by a cell membrane which allows the cell to selectively filter what can enter and leave the intracellular region. The main component of the membrane is a phospholipid bilayer which is embedded with transmembrane proteins that transport needed nutrients across the barrier. Nutrients can diffuse through the membrane proteins and some through the lipid bilayer itself. The breakdown of this system can lead to disease, cell necrosis, and even death of the 
organism. Understanding how cell membranes and proteins regulate these processes could advance potential applications in drug design or gene therapy. A clear grasp of the complex biologically relevant protein-rich bilayer systems is difficult without a fundamental understanding of the structure and dynamics of the simpler protein-free, hydrated lipid bilayer systems.

It is known that water layering on a hydrated lipid bilayer membrane alters the structural and dynamic properties of water near the membrane surface. One experimental method of studying such systems is quasielastic neutron scattering (QENS). Due to the low scattered neutron intensity, some experiments are carried out with thousands of membranes stacked on top of one another. In such systems it is impossible to control or even know how much water lies between the layers, or prevent bilayer-bilayer interactions, resulting in an inhomogeneous system that is difficult to model and simulate.

A more recent experimental method places a single membrane onto a substrate (e.g., silica), Fig. 3.1, and then stacks about a hundred of the solid-supported lipid membranes to build up sufficient scattered neutron intensity $[12,13]$. While this method removes bilayer-bilayer interactions it introduces bilayer-silica interactions which may affect both lipid and water structural and dynamic properties, at least in the proximity of the substrate.

Here we study, using molecular dynamics (MD) simulations, how certain properties of lipids and water are affected by the introduction of a silica substrate. Such a study requires multiple simulations. The first simulation, containing only a hydrated 1,2-Dimyristoyl-sn-glycero-3-phosphocholine (DMPC) bilayer, is used as a reference for the additional simulations that contain the silica substrate. The supported sys- 


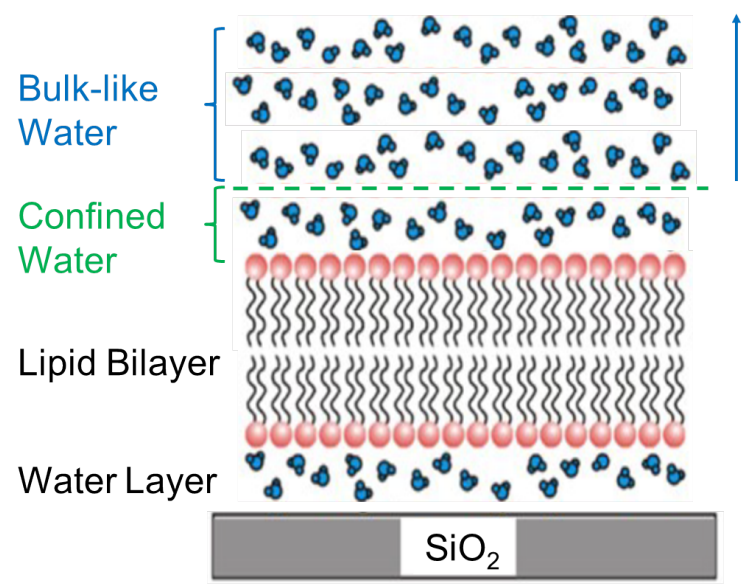

Figure 3.1: Schematic of a lipid bilayer mounted on a silica substrate. Courtesy of Dr. Taub's lab [13].

tems only differ in the amount of water between the silica substrate and the lower leaflet of the bilayer.

The simulations were used to compare the free-standing and supported bilayers and look for structural and dynamic changes. The simulation results were also compared to available experimental results when possible. Some of the questions that we have been trying to answer include: what is the water density profile around the lower bilayer leaflet (nearest to $\mathrm{SiO}_{2}$ ) and the upper leaflet; what is the thickness of the water layer between the lower leaflet and the substrate; does the introduction of the substrate change the number of water molecules that are bound to the lipid head group; are there changes to the diffusion coefficients of the hydration waters in and near the membrane; is the area per lipid affected by the support? 


\subsection{Methods}

\subsubsection{Bilayer Simulations}

All-atom MD simulations were run with the NAMD 2.10 simulation engine in the constant-volume, constant-temperature (NVT) ensemble with periodic boundary conditions [24]. Prior to the production runs, a 100 ns constant-pressure, constanttemperature (NPT) equilibration simulation was completed to determine the average $x$ and $y$ simulation box dimensions, parallel to the plane of the membrane. An additional simulation with the lateral box area, $A$, held constant (NPAT) was run to determine the average $z$ box dimension, perpendicular to the plane of the membrane.

For the subsequent NVT simulations, the $x$ and $y$ box dimensions were both equal to $62 \AA$, which gives an area per lipid of $60.1 \AA^{2}$, near the experimental value of 59.6-60.6 $\AA^{2}$ for fully hydrated, DMPC lipid vesicle bilayers under normal conditions [25-27]. In both the free-standing and supported simulations, the CHARMM36 force field [28] was employed for the DMPC lipid and water molecules, using the TIP3P water model. For the supported system, the water contact angle (WCA) force field was used for silica atoms [29].

The Particle Mesh Ewald summation method was employed for the long-range electrostatic interactions with grid spacing of $1 \AA$ [30]. For the remaining nonbonded interactions, we utilized the standard Lennard-Jones potentials where the interactions are cut off at $12 \AA$ with a smooth switching function turned on at $10 \AA$. The Langevin thermostat was used to control the temperature at $303 \mathrm{~K}$ with a damping coefficient

of $5 \mathrm{ps}^{-1}$. This value for the damping coefficient has been shown to produce the bulk water diffusion coefficient observed in experiment. The simulations employed a time 
step of $1 \mathrm{fs}$. The nonbonded and electrostatic interactions were calculated every 2 fs and $4 \mathrm{fs}$, respectively. The positions of the atoms were saved every 5 ps for each of the 150 ns production runs.

\subsubsection{Annealing Simulation of the Silica Substrate}

To construct a rectangular silica substrate with a pore through the center, we followed the procedure described in Ref. [31]. First, oxygen and silicon atoms were placed in a 2 to 1 ratio into a $62 \times 62 \times 60 \AA$ simulation box. The atoms interacted only through nonbonded forces defined in the van Beest, Kramer and van Santen (BKS) force field [32] with the Vollmayr modification [33].

To add a pore through the silica slab, atoms were removed from the desired pore region and an additional user-defined grid was employed that repulses atoms when they enter the pore region. Grid-steered molecular dynamics was used with the repulsion force defined in Eq. (3.1), with the constants $R=3 \AA, \sigma=3 \AA$, and $F_{0}=8$ $\mathrm{kcal} / \mathrm{mol} / \AA$, where $r_{i j}$ is the distance between the silicon or oxygen atom and the grid point in the pore.

$$
F_{i j}= \begin{cases}F_{0} \mathbf{e}_{i j} & r_{i j} \leq R \\ F_{0}\left(1-\left(r_{i j}-R\right) / \sigma\right) \mathbf{e}_{i j} & R<r_{i j}<R+\sigma \\ 0 & \text { otherwise }\end{cases}
$$

The annealing simulation was carried out in a NVT ensemble with periodic boundaries conditions. The Langevin thermostat was used to control the temperature with a damping coefficient of $5 \mathrm{ps}^{-1}$. For the nonbonded interactions, a cutoff distance of 
Table 3.1: Silica WCA force field parameter values.

\begin{tabular}{cccc}
\hline $\mathrm{i}$ & $\epsilon_{i}^{\min }$ & $r_{i}^{\min }$ & $q_{i}$ \\
\hline $\mathrm{Si}$ & -0.30 & 2.1475 & 1.0 \\
$\mathrm{O}$ & -0.15 & 1.75 & -0.5 \\
\hline
\end{tabular}

$5.5 \AA$ was employed and the integration time step was $1 \mathrm{fs}$.

The system was annealed using grid-steered molecular dynamics [34] in four stages: temperatures (and time durations) were $7000 \mathrm{~K}$ (20 ps), $5000 \mathrm{~K}$ (20 ps), $2000 \mathrm{~K}$ (50 ps) and $300 \mathrm{~K}(50 \mathrm{ps})$. After annealing, the substrate was cleaved to a $z$ thickness of $\sim 25 \AA$. This procedure produced a silica substrate with the experimental bulk mass density $\left(2.2 \mathrm{~g} / \mathrm{cm}^{3}\right)$, surfaces with low surface roughness, and a pore through the center along $\hat{z}$. To prevent changes to the silica mass density and to inhibit an excessive amount of water from entering the silica, the silica substrate atoms were restrained with a harmonic spring constant of $20 \mathrm{kcal} / \mathrm{mol} / \AA^{2}$.

Additionally, the bond energy term for Si-O bonds was $k_{b}=1.0 \mathrm{kcal} / \mathrm{mol} / \AA^{2}$ and the equilibrium distance was $b_{0}=1.6 \AA$, where the silicon to oxygen bonds were only created between atoms that were within $2 \AA$ of each other after annealing. The silica atom nonbonded CHARMM-like force field parameter values, listed in Table 3.1, were chosen for their ability to replicate experimental water contact angles on silica substrates [29]. We chose to use the WCA force field for its ability to reproduce the known hydrophilic properties of silica without the need of adding explicit hydroxyl groups on its surface. The CHARMM combining rules were employed for interactions between silica and non-silica atoms in subsequent supported bilayer simulations. 


\subsection{Results}

\subsubsection{Bilayer: free-standing}

For the free-standing (FS) bilayer, the starting atomic positions were taken from the end of a previous simulation [15]. Additional water was added on each side of the bilayer to guarantee that the leaflets and their hydration waters did not interact across the periodic $z$ boundary. Our system consists of a total of 37,001 atoms, with 128 lipids (15,104 lipid atoms) and 7299 water molecules in a simulation box with dimensions of $62 \AA$ in $x$ and $y$ directions and $91 \AA$ in the $z$ direction, Fig. 3.2, well above the 28-29 waters per lipid necessary for full hydration of the membrane [26].
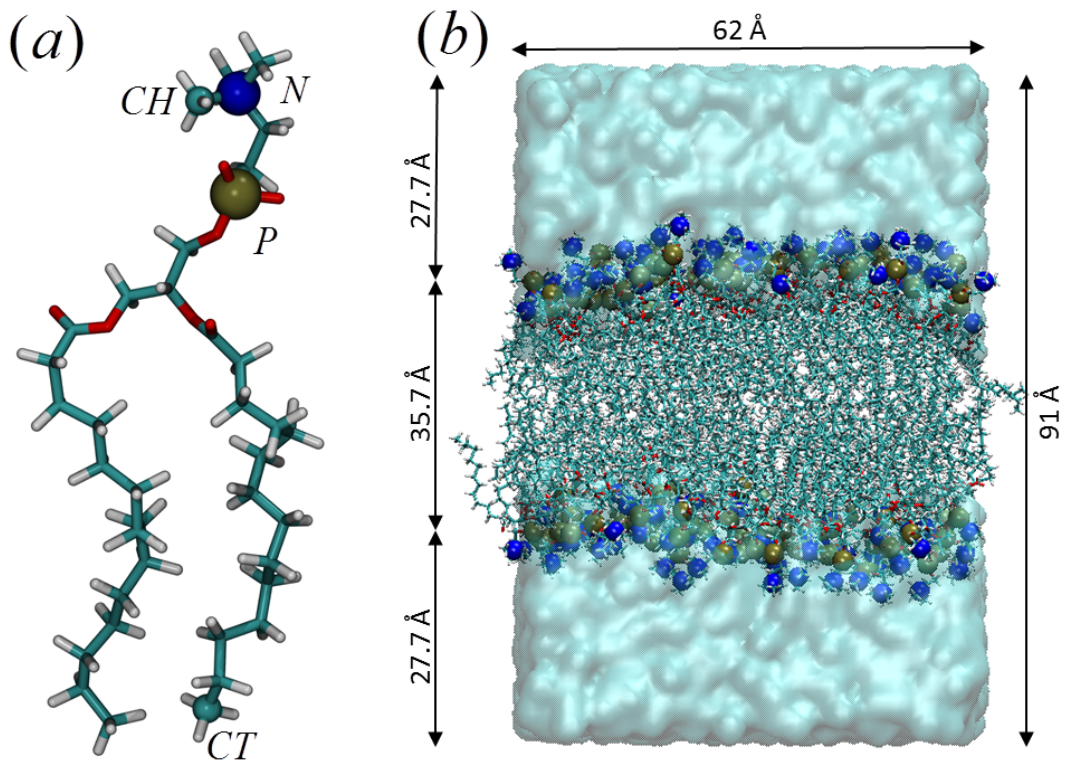

Figure 3.2: (a) Snapshot of a DMPC lipid in licorice representation with spheres representing atoms of interest: phosphorus (tan), nitrogen (blue), headgroup carbon $(\mathrm{CH}$, teal), and tail carbon $(\mathrm{CT}$, teal). (b) A free-standing lipid bilayer snapshot displaying the phosphorus and nitrogen atoms, water above and below the membrane, and the lipid tails in licorice representation. The simulation box dimensions along with the bilayer and water layer thicknesses are also provided. 
From both the pictorial representation, Fig. 3.2, and the number density profiles, Fig. 3.3, we can see that a "surface" of the membrane is difficult to define due to the relatively large out-of-plane motion of the lipid atoms. In this study, we have defined the bilayer surface to be the mean $z$ coordinate of the phosphorus atoms in each leaflet, $\left\langle z_{p}\right\rangle$, which is marked by the dashed vertical lines in Fig. 3.3. The center of the membrane is at the origin $(z=0 \AA)$.

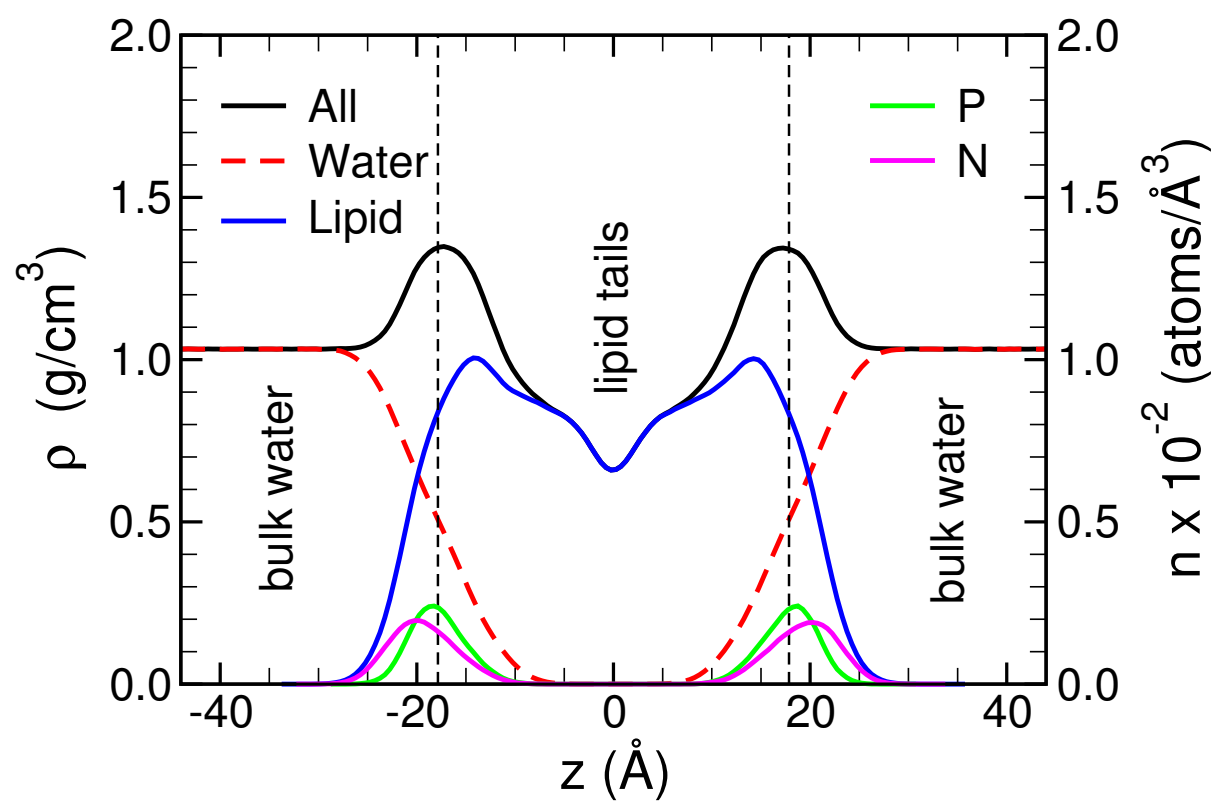

Figure 3.3: Free-standing bilayer mass density profiles for all atoms (black), lipids (blue), and water (red) as well as number density profiles for the phosphorus (green) and nitrogen (pink) atoms in the lipid headgroups. Vertical dashed lines mark the mean $z$ coordinate for the phosphorus atoms in each leaflet.

The plot also shows a large overlap of the water and lipid mass density profiles, from $7 \AA \leq|z| \leq 28 \AA$, which demonstrates that water penetrates deep into the bilayer where the water can interact with the slower moving lipids. We refer to the water in these regions as hydration water. The structure and dynamics of the hydration water and its interaction with the lipids has been studied extensively with a variety 
of experimental techniques [12,13,25,35-39] and MD simulations [14, 15,40-44]. Our first MD simulation presented below did reproduce some of the previous results for an FS membrane and were used to make comparisons between our FS and supported lipid membranes.

\subsubsection{Silica Substrate}

The annealed substrate was hydrated and a 10 ns NVT simulation was run to equilibrate the system. The equilibrium water mass density profile is shown in Fig. 3.4. The increased mass density is due to the water molecules that have been adsorbed on the surface of the silica.

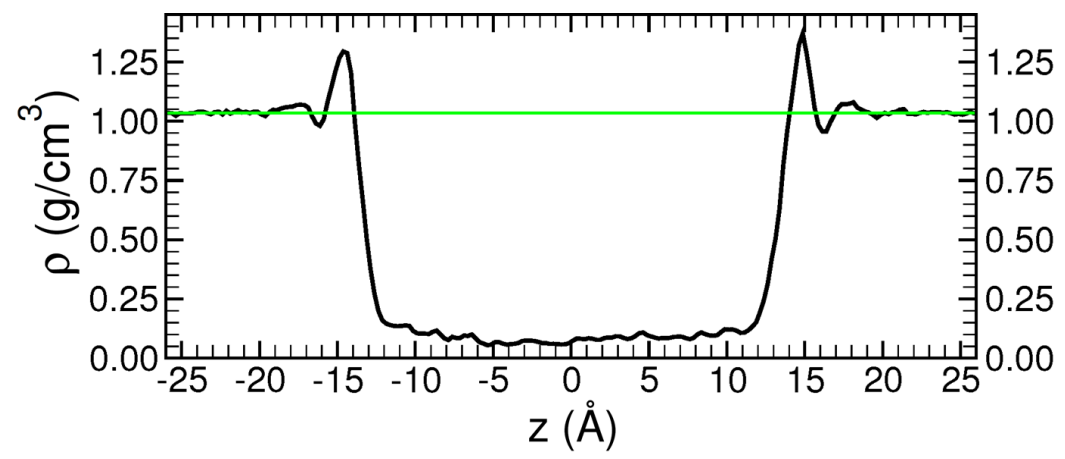

Figure 3.4: Water mass density profiles for the hydrated silica substrate. The center of the silica substrate is located at $z=0 \AA$. The nonzero water density in the silica was due to the water filled pore region. Water is adsorbed onto the silica surface but quickly goes to the bulk value $\left(1.03 \mathrm{~g} / \mathrm{cm}^{3}\right)$ away from the surface.

\subsubsection{Bilayer: supported}

The hydrated bilayer and silica systems were combined to create a supported (SP) bilayer system which has 128 lipids (15,104 lipid atoms), 8337 water molecules (25,011 
atoms), 6600 silica atoms (2200 silicon, 4400 oxygen), for a total of 46,715 atoms. The simulation box size was $62 \AA \times 62 \AA \times 122 \AA$, Fig. 3.5.
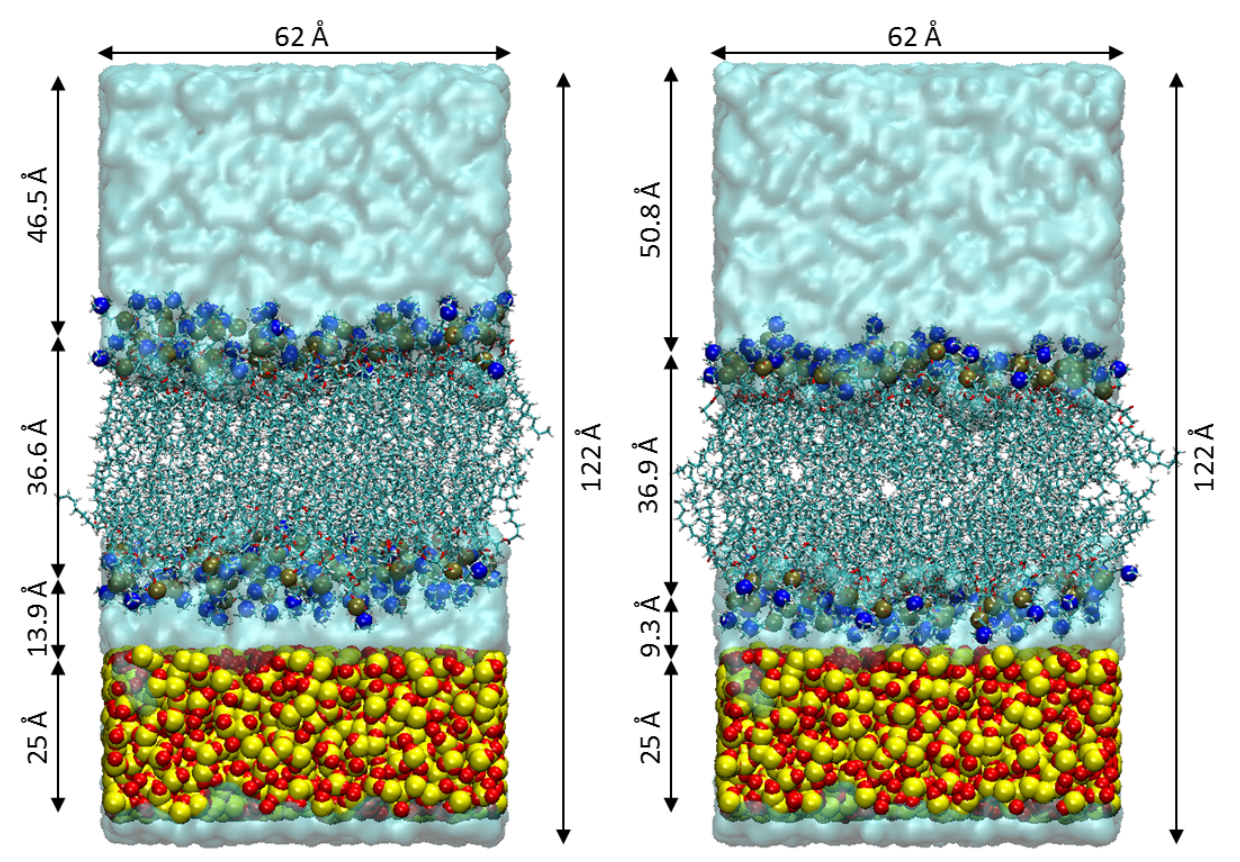

Figure 3.5: Snapshot of SP14 (left) and SP9 (right) with the nitrogen atoms (blue spheres), phosphorus atoms (tan spheres), the silica oxygen (red) and silicon (yellow) atoms, water above and below the bilayer, and the lipid tails in licorice representation. Fig. 3.6 is another representation of SP14, but with different atoms removed to more clearly differentiate between components of the system.

The only difference between our supported simulation setups is the different distances between the silica and the lower leaflet of the membrane. This distance is defined as

$$
z^{\prime}=\left\langle z_{p}\right\rangle_{\text {lower }}-z_{\text {silica }, 50 \%}
$$

where $\left\langle z_{p}\right\rangle_{\text {lower }}$ is the the mean position of the phosphorus atoms in the lower leaflet, and $z_{\text {silica }, 50 \%}$ is the $z$ coordinate where the silica mass density is $50 \%$ of the bulk 


\begin{tabular}{|c|c|c|}
\hline (a) 46,715 total atoms & $\begin{array}{c}\text { (b) } 64 \text { lipids/leaflet } \\
15104 \text { lipid atoms } \\
6600 \text { silica atoms }\end{array}$ & $(C)$ \\
\hline 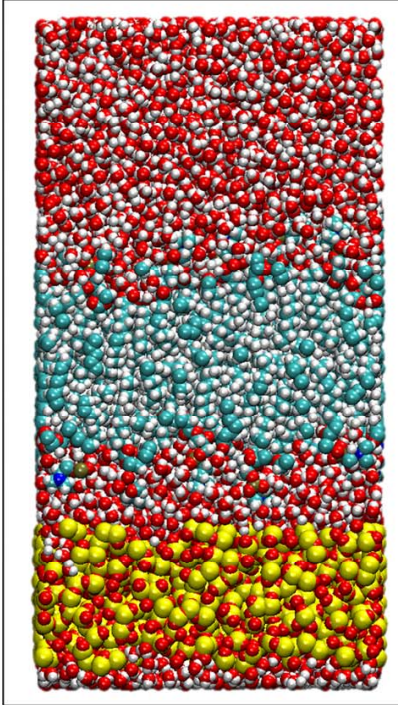 & 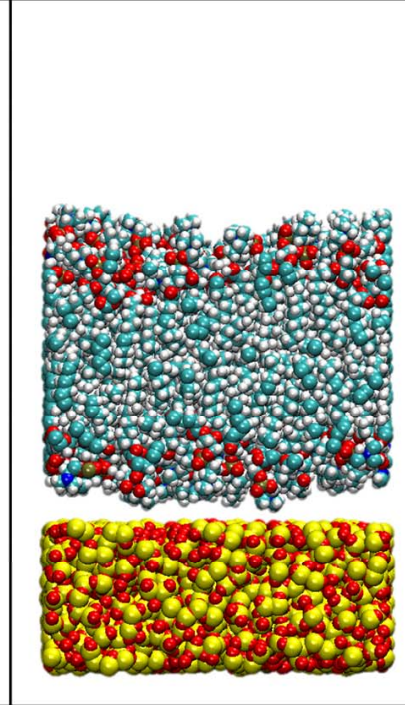 & 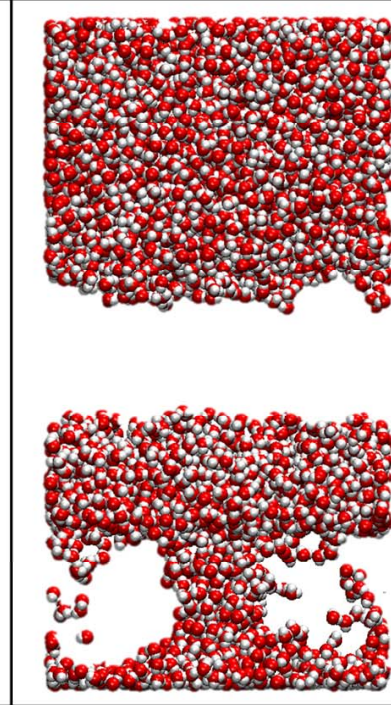 \\
\hline
\end{tabular}

Figure 3.6: Snapshots of SP14 with (a) all the atoms, (b) without water, and (c) only water. The water molecules can diffuse through the silica pore to equilibrate the pressure above and below the bilayer.

silica value.

We refer to one simulation of this system, also shown in Fig. 3.6a, as SP14, because the distance between the silica and the bilayer was $\sim 14 \AA$ (left snapshot in Fig. 3.5). In Fig. 3.6b, the water molecules have been removed to visualize the $14 \AA$ distance, i.e., the lower leaflet of the bilayer to the silica surface. Even in this small region, $62 \AA \times 62 \AA \times 14 \AA$ there are still thousands of water molecules present that hydrate both the silica surface and the lipid headgroups. When the silica and lipid atoms are hidden, the waters penetrating into the lipid headgroup region and into the silica pore are clearly visible, Fig. 3.6c.

The first supported NVT simulation began with $z^{\prime}=16 \AA$ and no restraints on the bilayer and with the water free to move in either direction through the silica pore. 


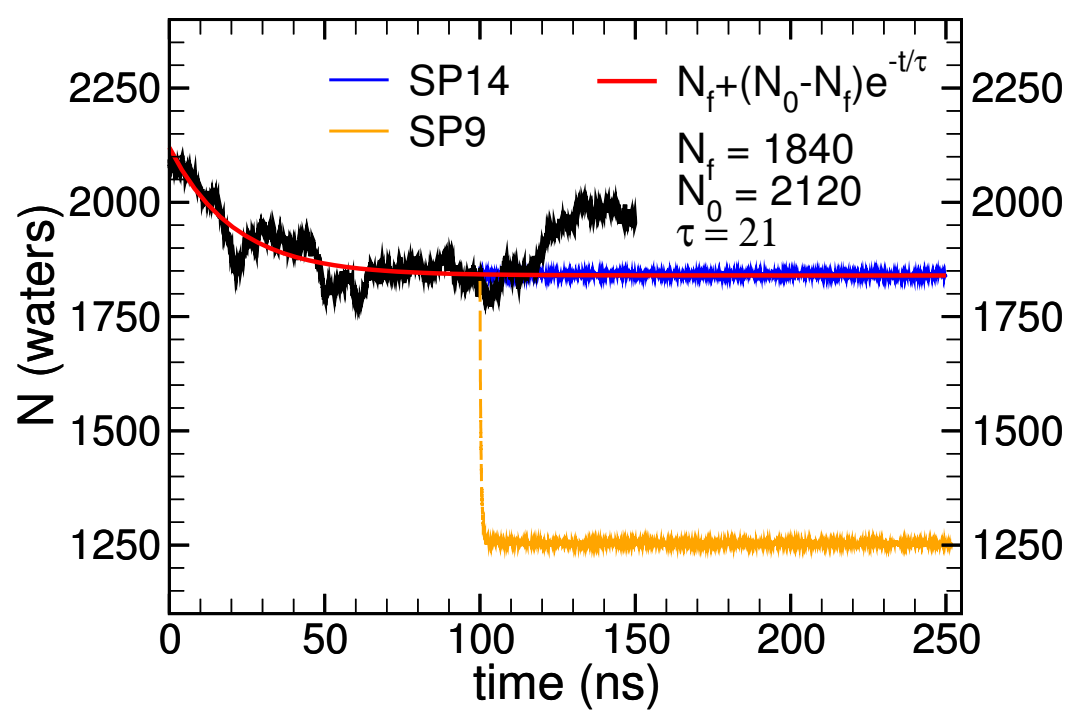

Figure 3.7: The time evolution of the number of water molecules between the center of the membrane and the silica substrate. In the first simulation (black), water could move through the pore and the bilayer was free to move in the $\hat{z}$ direction. The second (blue, SP14) and third (orange, SP9) simulations restrained the waters in the silica pore to enforce $z^{\prime}=13.9 \AA$ and $z^{\prime}=9.3 \AA$, respectively. Also shown (orange dashed) is a simulation where the water was drained through the pore.

Consequently, the membrane could determine the separation between itself and the silica by moving up or down, while water could filter in and out to cancel any pressure differences arising from the vertical motion of the bilayer.

During the first $50 \mathrm{~ns}$, the bilayer moved towards the silica and then remained near $z^{\prime}=13.9 \approx 14 \AA$. For $50 \leq t \leq 100$ ns, about 1840 water molecules occupied the region between the membrane and silica. It appeared that the bilayer had found an equilibrium $z^{\prime}$, but during an additional $50 \mathrm{~ns}$ simulation the bilayer moved away from the support by about $2 \AA$. The time evolution of the number of water molecules between the silica and the lower leaflet of the bilayer is shown in Fig. 3.7. While it is possible that on a microsecond timescale the bilayer may find an equilibrium $z^{\prime}$, we are not able to probe this possibility with our computational resources. 
Assuming that the lipid and water structure and dynamics are most greatly affected by the presence of the silica substrate at the smallest separation distance, we chose to start another simulation at $z^{\prime}=13.9 \AA$. Taking the coordinates from the previous simulation at $t=100 \mathrm{~ns}$, we began a new simulation but now with the water molecules in the silica pore harmonically restrained with a $20 \mathrm{kcal} / \mathrm{mol} / \AA^{2}$ force constant to prevent water from passing through the pore. With the separation distance now enforced at $z^{\prime}=13.9 \AA$, another $150 \mathrm{~ns}$ simulation was run. As presented below, the resulting analysis revealed few differences between the hydrated FS lipid bilayer and the SP14 system.

Because little difference was found between SP14 and FS, water was then drained through the silica pore to further decrease the water layer thickness between the membrane and the silica. By running a 2 ns Steered Molecular Dynamics simulation [34] (in which the water oxygen atoms in the silica pore region were subjected to an additional constant force of $0.4 \mathrm{kcal} / \mathrm{mol} / \AA$ in the $-\hat{z}$ direction), the water thickness was further reduced. During the simulation about 600 waters were drained through the pore. Afterward, the harmonic restraints on the pore waters were reintroduced to enforce $z^{\prime}=9.3 \AA$ for another $150 \mathrm{~ns}$ simulation. This new system is referred to as SP9.

\section{Bilayer Thickness and Area Per Lipid}

The QENS experiments mentioned previously stack about a hundred lipid bilayer coated silica wafers that are in a sealed can after deposition of a droplet of water. To hydrate the membranes, the water has to evaporate and come into contact with the bilayer. To observe the effects of low hydration, the bilayer thicknesses of the 


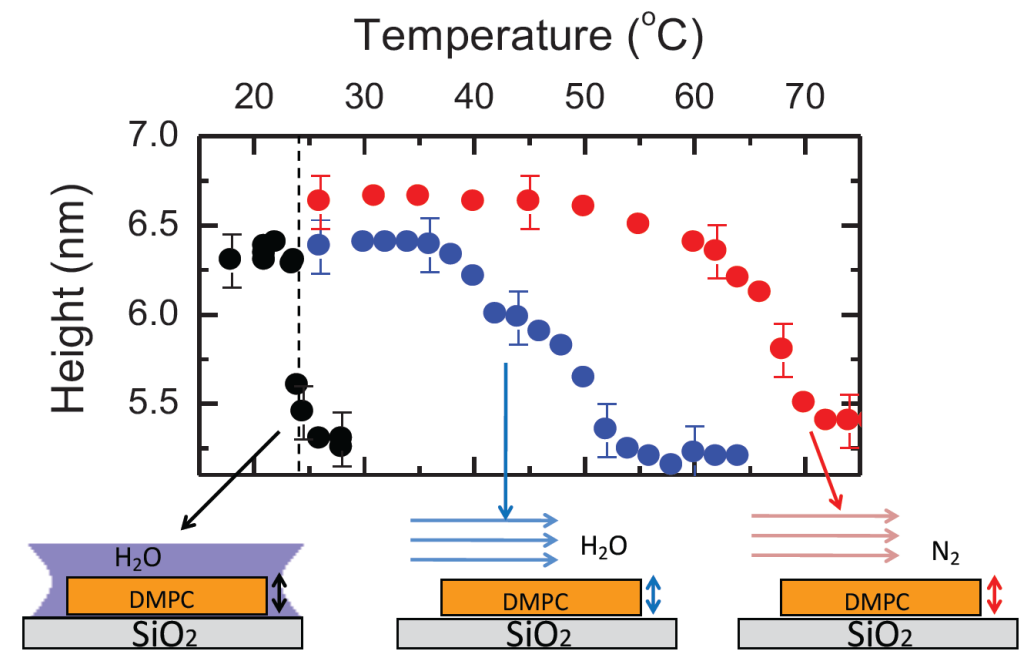

Figure 3.8: AFM measurement of the height of a lipid bilayer on a silica substrate. The experiment was carried out in a fully hydrated system (black), when flowing humid air over the bilayer (blue), and flowing nitrogen over the membrane (red). Courtesy of Dr. Taub's lab [45].

supported DMPC lipid bilayers were measured using Atomic Force Microscopy (AFM) in cases where humid air flows over the bilayer and when the bilayer is fully submerged in water.

The AFM results show that the distance from the top of the membrane to the substrate drops significantly near the normal gel-to-fluid phase transition temperature, $296 \mathrm{~K}[46,47]$, for the fully hydrated system, Fig. 3.8. When humid air flowed over the bilayer, the measured height also decreased but beginning at a higher temperature, near $310 \mathrm{~K}$. The transition to a smaller height occurred over a range of temperatures extending to $325 \mathrm{~K}$. From these measurements it was inferred that the phase transition temperature shifted to a higher temperature due to the low-hydration environment and/or the presence of the substrate. Therefore, at lower levels of hydration, the supported membrane appears to be in the gel phase at our simulation temperature, 
$T=303 \mathrm{~K}$, while in our MD simulations at this temperature the lipid bilayer is in the fluid phase.

MD simulations of free-standing bilayers at differing levels of hydration have been carried out previously [48]. They showed that for POPC lipid bilayers, when the hydration is decreased from 28 waters/lipid (full hydration) to 11.7 and 5.4, then the area per lipid (APL) decreased respectively by $25 \%$ and $30 \%$, while the bilayer thickness increased by $34 \%$ and $42 \%$. It is important to note that lipid forcefield parameters are parameterized at full hydration. These low hydration simulations were equilibrated for $400 \mathrm{~ns}$ each to verify that the bilayers did not disintegrate [48], which had occurred in prior attempts to simulate low hydration bilayers [49].

We employed constant-volume MD simulations (imposed by the rigidity of the silica substrate) in which the APL could not be changed. Several other properties (bilayer thickness, acyl chain order parameter, surface tension) were monitored to see if a change in APL would be expected if the volume was not constant. To determine if the membrane thickness changed with the introduction of the substrate, we calculated the mean $z$ coordinate of the lipid phosphorus atoms and of the lipid headgroup (HG) center-of mass for each leaflet and then their difference,

$$
\Delta z=\left\langle z_{i}\right\rangle_{\text {upper }}-\left\langle z_{i}\right\rangle_{\text {lower }}
$$

The bilayer thickness results are shown in Table 3.2 and Fig. 3.9. Because the results show that both the phosphorus and HG center-of-mass follow the same trend, only the phosphorus results will be provided from here on.

There is a small increase in the bilayer thickness when the silica is introduced and the membrane is brought closer to the surface. The increase from $35.7 \AA$ to $36.6 \AA$ to 

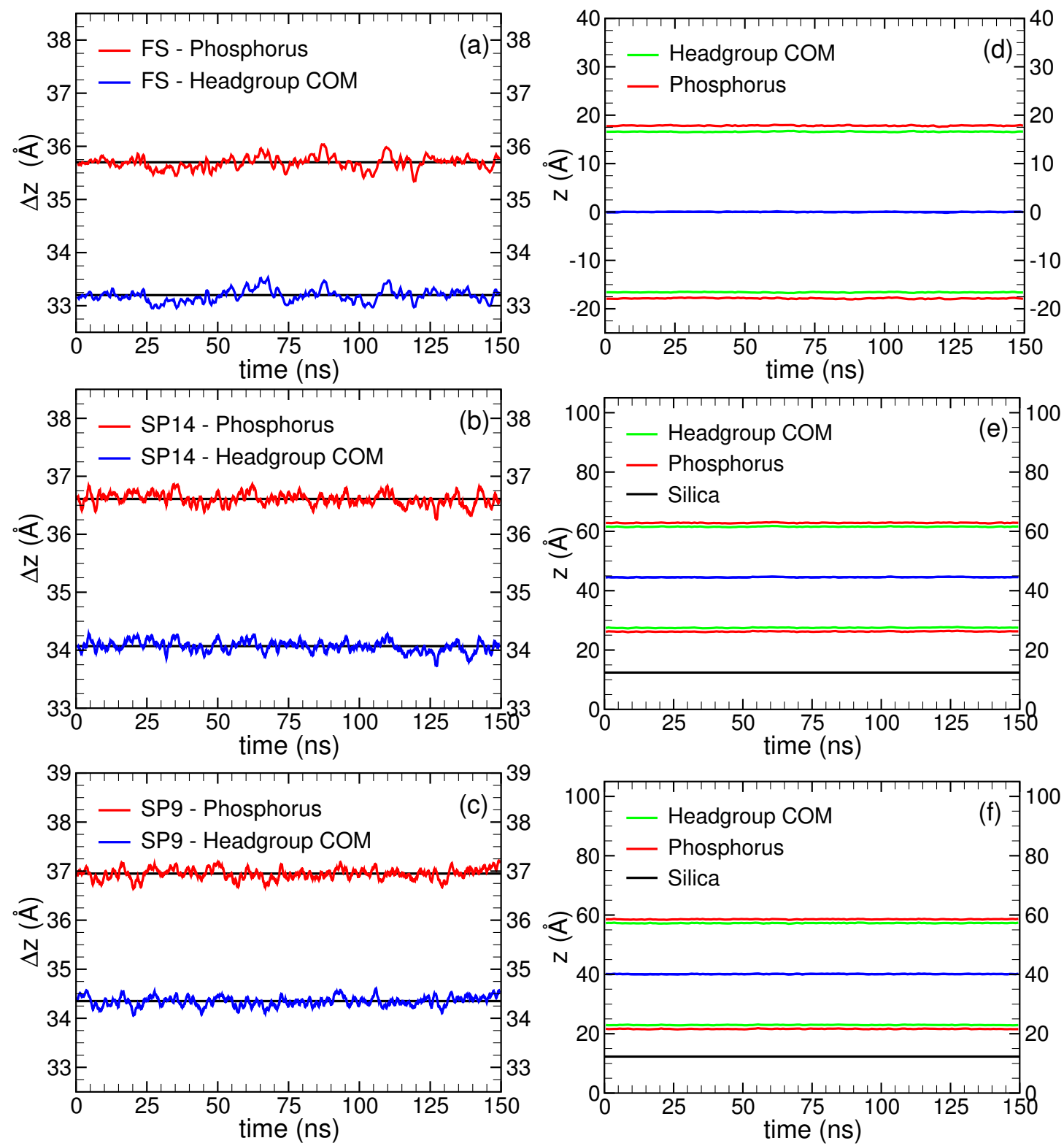

Figure 3.9: (a-c) Time evolution of the bilayer thickness, $\Delta z$, measured as the difference between the mean phosphorus, and headgroup center-of-mass, $z$ coordinate values in each leaflet. (d-f) The time evolution of the mean $z$ position of the phosphorus and lipid headgroup atoms for each leaflet with respect to the silica support (black line in (e) and (f) at $z=12.3 \AA$ ). The P-P bilayer thicknesses and standard deviations were $35.7 \pm 0.18 \AA$ (FS), $36.6 \pm 0.18 \AA$ (SP14) and $36.9 \pm 0.19 \AA$ (SP9). The silica to lower leaflet (mean phosphorus) distances were $13.9 \AA$ (SP14) and 9.3 $\AA$ (SP9). 


\begin{tabular}{ccc}
\multicolumn{3}{c}{ Table 3.2: Bilayer Thickness } \\
\hline \hline Simulation & $\Delta z_{P-P}(\AA)$ & $\Delta z_{H G-H G}(\AA)$ \\
\hline FS & $35.7 \pm 0.18$ & $33.2 \pm 0.17$ \\
SP14 & $36.6 \pm 0.18$ & $34.1 \pm 0.17$ \\
SP9 & $36.9 \pm 0.19$ & $34.3 \pm 0.17$ \\
\hline
\end{tabular}

$36.9 \AA$ is much smaller than what was seen in the AFM experimental results shown in Fig. 3.8, where the supported bilayer thickness measurement was about 20-25\% smaller at $303 \mathrm{~K}$ for the system that was fully-hydrated versus the low-hydration environment. This may be due to the fixed APL or the full hydration of the bilayer in all simulations.

\section{Acyl Chain Order Parameter}

Another lipid property that would change if the bilayer had undergone a fluid-to-gel phase transition is the acyl chain (lipid tail) order parameter which is related to the angle, $\theta_{i}$, between each carbon to hydrogen bond vector, $C_{i}-H_{i}$, and the membrane normal, and is calculated as

$$
S_{C D}^{i}=\frac{1}{2}\left\langle 3 \cos ^{2} \theta_{i}-1\right\rangle
$$

Here the $C_{2}$ atom is near the top of the lipid tail, and the $C_{14}$ is the terminal carbon. When the bilayer goes from the liquid to gel phase the tails straighten out and the order parameter increases. The results for our MD simulations (see Fig. 3.10) do not show any significant or systematic change from FS to SP14 and SP9, i.e., the membrane remains in the fluid phase. 

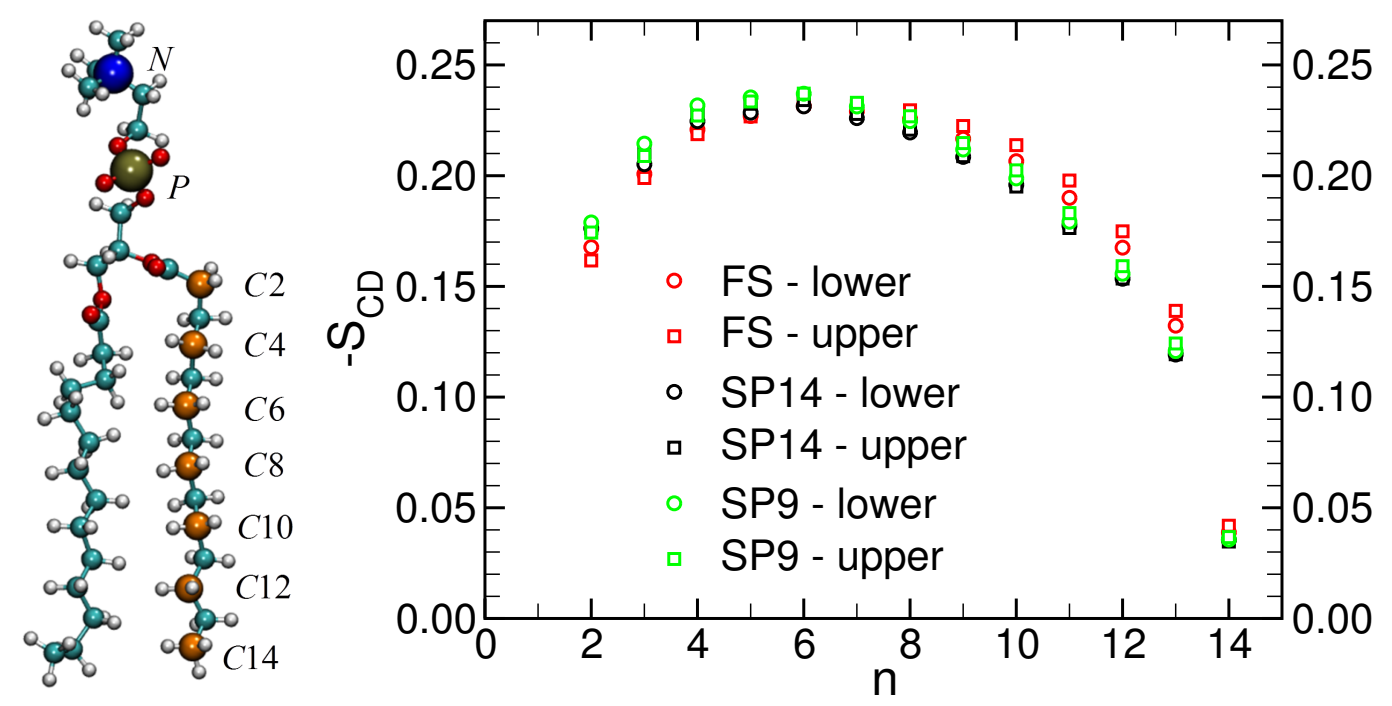

Figure 3.10: Acyl chain (lipid tail) order parameter as a function of the carbon atom index. The values have changed slightly due to the introduction of the substrate, with an increase in values near the top of the tails and decrease near the ends. On the left, a lipid representation with the even numbered carbon tail atoms (colored with orange spheres) is shown for one of the tails.

\section{Hydrophilic Layer Thickness}

An alternative method of determining the thickness of the water layer below the membrane is to measure the hydrophilic layer thickness (HLT), which is defined as the distance from where the silica mass density drops to $50 \%$ of its bulk value to where the lipid tail mass density is $50 \%$ of its maximum. Neutron reflectivity measurements have been able to estimate this thickness to be $14.6 \AA$ for a hydrated POPC lipid bilayer supported on a silica substrate [50].

POPC and DMPC lipids have an identical headgroup, hence identical hydrophilic regions. Because the lipids only differ with respect to the lengths of their tails, this measurement should be the same as for our DMPC-silica system. The HLT distance during the supported bilayer simulations were $14.3 \AA$ and $19.0 \AA$, respectively for SP9 


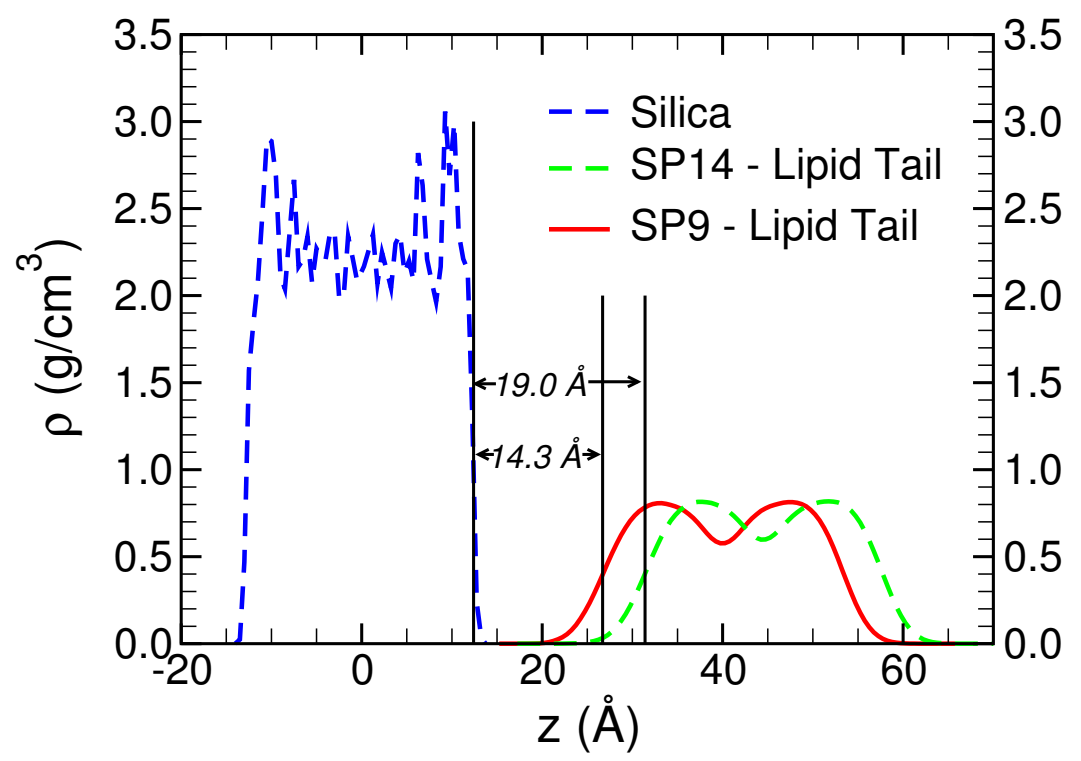

Figure 3.11: Mass density profiles for the silica and lipid acyl chains (tails) in the supported membrane systems. The hydrophilic layer thicknesses for SP9 and SP14 were $14.3 \AA$ and $19.0 \AA$, respectively.

and SP14, Table 3.3 and Fig. 3.11. HLT is $\sim 5 \AA$ larger than $z^{\prime}$.

Table 3.3: Water Layer Thickness

\begin{tabular}{cccc}
\hline \hline Name & Type & $z^{\prime}(\AA)$ & HLT $(\AA)$ \\
\hline FS & Free-Standing & - & - \\
SP14 & Supported & 13.9 & 19.0 \\
SP9 & Supported & 9.3 & 14.3 \\
\hline
\end{tabular}

\section{Membrane Surface Tension}

To determine the surface tension $\sigma$ of a hydrated lipid bilayer, the simulation box was divided up into $2 \AA$ thick slabs in the $\hat{z}$ direction and the components of the pressure tensor through each slab surface were calculated. The lateral (tangential) components of the pressure tensor, $P_{x x}$ and $P_{y y}$, were averaged, $P_{\|}=\left(P_{x x}+P_{y y}\right) / 2$, while the normal component along $\hat{z}$ is $P_{\perp}=P_{z z}$. The surface tension is given by [51], 


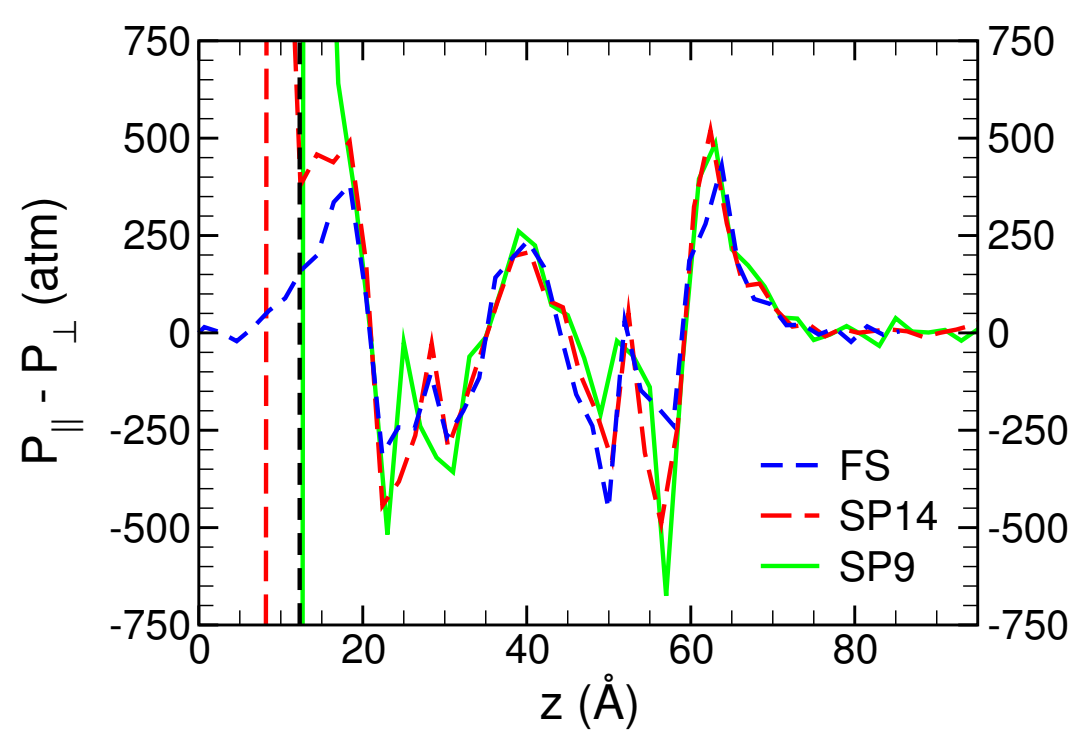

Figure 3.12: The pressure tensor difference profile as a function of the $z$ coordinate value. The surface tension is obtained by integrating over the entire region. In the supported systems (SP9 and SP14), the lower leaflet interacts with water molecules at the silica surface which increases the integrated $P_{\|}-P_{\perp}$. The FS and SP14 profiles have been shifted to align them with the peak near $z=40 \AA$ in SP9. The $z$ coordinate value of the silica surface (black dashed vertical line) has been drawn at $z=12.3 \AA$.

$$
\sigma=-\int_{-z_{b o x} / 2}^{z_{b o x} / 2}\left(P_{\|}-P_{\perp}\right) d z
$$

In general, free-standing hydrated lipid bilayers have a small positive surface tension $[52,53]$. By contrast, in our supported systems, the silica substrate-water interfacial tension has a large negative value.

Integrating over the entire simulation box for the FS bilayer gives $\sigma=8.6 \mathrm{dyn} / \mathrm{cm}$ which is similar to previous MD simulations [54]. Note that there are two lipid-water interfaces for each bilayer, so the surface tension on each leaflet lipid-water interface is half of the reported value. For the supported systems, the silica complicates the calculation as we cannot integrate over the entire box as this would include the 
substrate. Integrating only from the silica surface to the bulk water region one obtains for the surface tension $\sigma=95.1 \mathrm{dyn} / \mathrm{cm}$ and $\sigma=125.6 \mathrm{dyn} / \mathrm{cm}$ for SP14 and SP9, respectively. Note that this surface tension includes both the upper and lower surface of the membrane and the water on the top surface of the silica substrate.

When the membrane is closer to the substrate, the lateral pressure profile is altered significantly (Fig. 3.12) due to the presence of the substrate, thus leading to a substantial increase of the surface tension of the lower leaflet. Consequently, if the simulation box lateral dimensions were flexible, this could lead to a contraction of the membrane and a decrease of the APL. Thus, it appears that the presence of the silica substrate may cause a reduction of the APL in the lower leaflet, which could increase the thickness of the bilayer and trigger its transition to the gel phase.

\section{Out-of-Plane Lipid Fluctuations}

Another way to determine the bilayer thickness, and to see how the substrate affects membrane surface dynamics, is to monitor the out-of-plane lipid fluctuations by calculating the probability distribution of the $z$ coordinate values of the lipid phosphorus atoms, Fig. 3.13. A Gaussian function was used to fit each distribution with the resulting full-width at half $\max ($ FWHM) being 6.6 (6.5) $\AA$ for the upper (lower) leaflet in the FS system, and 5.4 (6.1) $\AA$ and 5.4 (6.3) $\AA$ for SP14 and SP9, respectively.

For both of the supported systems, the FWHM decreases but interestingly the upper leaflets saw a larger decrease. The Gaussian fitting method becomes less meaningful as the distributions lose their Gaussian shape. This is especially the case for the lower leaflet in SP9 where the lipid atoms are closest to the silica, near $z=5 \AA$ in Fig. 3.13c. So when a bilayer is moved sufficiently close to a supporting substrate, 

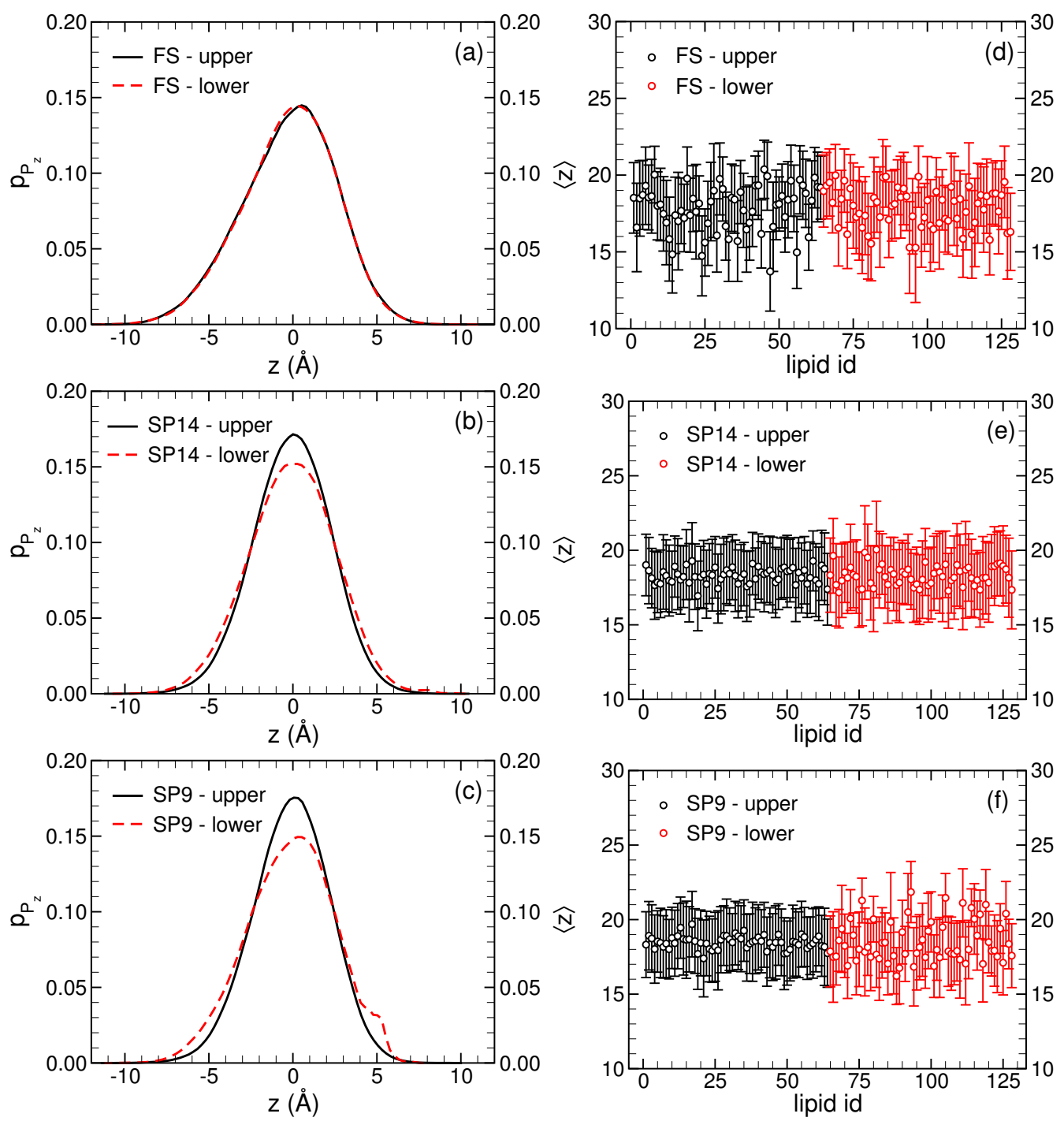

Figure 3.13: (a-c) Probability densities were determined by binning each lipid phosphorus atom by their $z$ coordinate values. Fitting the profile with a Gaussian function returned a FWHM of 6.6 (6.5) $\AA$ for FS, 5.4 (6.1) $\AA$ for SP14, and 5.4 (6.3) $\AA$ for SP9, for the upper (lower) leaflet. The distributions were aligned using the peak centers determined from fitting and by multiplying the $z$ values by -1 for the lower leaflet, meaning the lipid tails all point in the negative $z$ direction. Note that the lower leaflet in SP9 (c) loses its Gaussian shape. (d-f) The time-averaged $z$ coordinate values for each lipid phosphorus atom and standard deviation, shown as an error bar. The ensemble average and average standard deviation for the upper (lower) leaflet are 17.8 (17.8) $\AA$ and $2.4(2.4) \AA$ for FS, 18.3 (18.3) $\AA$ and 2.3 (2.5) $\AA$ for SP14, and $18.5(18.5) \AA$ and $2.2(2.2) \AA$ for SP9. 


\begin{tabular}{ccccc}
\multicolumn{4}{c}{ Table 3.4: Out-of-Plane Lipid Fluctuations } \\
\hline \hline & Upper Leaflet & \multicolumn{2}{c}{ Lower Leaflet } \\
Simulation & Mean $(\AA)$ & STD $(\AA)$ & Mean $(\AA)$ & STD $(\AA)$ \\
\hline FS & 17.8 & 2.4 & -17.8 & 2.4 \\
SP14 & 18.3 & 2.3 & -18.3 & 2.5 \\
SP9 & 18.5 & 2.2 & -18.5 & 2.2 \\
\hline
\end{tabular}

this fitting method fails.

A better approach is to calculate the time average and standard deviation of the $z$ position of the phosphorus atom for each individual lipid. With the time-averaged position and standard deviation we can determine the mean over the ensemble for each leaflet. In our simulations, the ensemble mean and standard deviation, where each bilayer center has been moved to $z=0 \AA$ are summarized in Table 3.4.

With this method of analysis, there is no systematic change from FS to SP14 as the the standard deviation increases (decreases) by $0.1 \AA$ for the lower (upper) leaflet. The change is systematic from FS to SP9 as the standard deviation for both the upper and lower leaflets decreased by $0.2 \AA$. As a note, the standard deviation values do increase with the simulation length, generally leveling off only after about $t=100 \mathrm{~ns}$.

\section{Mass Density Profiles}

As stated above for the hydrated FS bilayer, and shown in Fig. 3.3, water permeates deep into the lipid headgroup region. The mass density profiles for water and lipids overlap in the $7 \AA \leq|z| \leq 28 \AA$ region for each leaflet. Additionally, near the silica surfaces the water molecules have a maximum mass density of $\rho_{w}=1.24 \mathrm{~g} / \mathrm{cm}^{3}$, well above the bulk value of $\rho_{w, b u l k}=1.03 \mathrm{~g} / \mathrm{cm}^{3}$. The increased water mass density is 

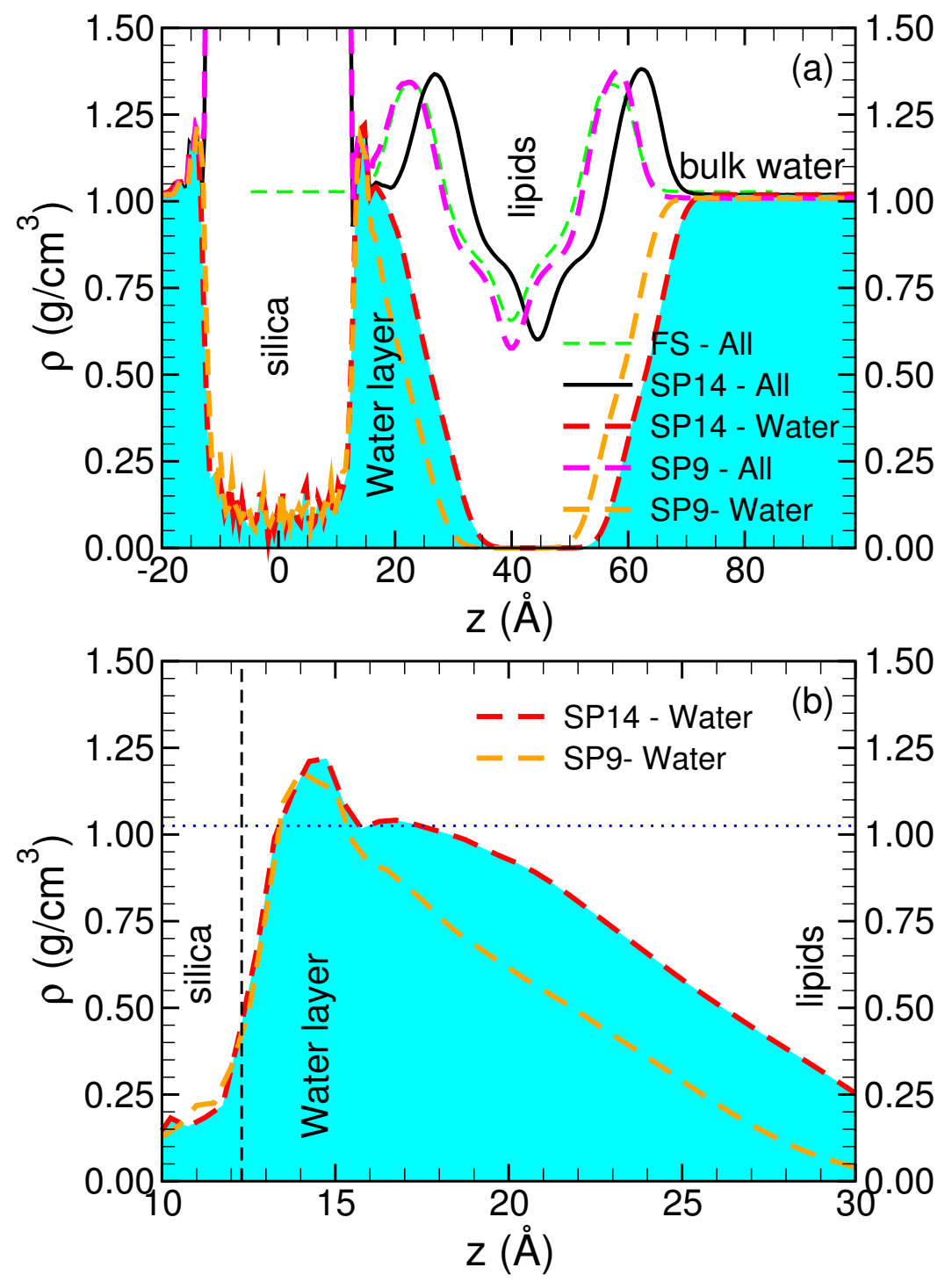

Figure 3.14: (a) All-atom mass density profiles for each simulation and water mass density profiles for the supported systems. The water profiles are included to visualize how the water layer decreases with decreasing separation between the bilayer and the silica. (b) The same mass density plot as (a) but for the $10 \AA \leq z \leq 30 \AA$ region, with the all-atom profiles removed to visualize better the hydration water layer on the lower leaflet and adsorption water layer on the silica. The silica surface (vertical black dashed line) and bulk water mass density value (horizontal blue dotted) have also been included. 
due to the high hydrophilicity of the silica surface.

As long as the membrane and silica surfaces are fully hydrated and have a minimum separation distance between them, each system remains independent as any additional water would behave as bulk water, and not hydration water. As the separation distance decreases and the bulk water is no longer present, then each surface competes for the water molecules, with the intermediate water molecules attracted to both surfaces, which acts as a sort of glue holding the bilayer to the silica substrate. This may explain the good adhesion of lipid membranes to silica substrates.

\section{Radial Distribution Function}

Results from neutron scattering experiments are able to determine the number of water molecules that are moving on the same time scale as the lipid atoms, which are called bound waters. Experiments determined the number of bound water molecules at 7-10 waters (QENS) [12], 9.7 waters (NMR) [55], and 5-6 waters [56].

The number of water molecules that are 'bound' to each lipid in simulation can be determined by counting the number of waters in the hydration shells around the lipid phosphorus atoms. Summing the waters into two bins, where the boundaries have been defined by the peak minima provides a quantitative measure of the number of waters bound to each lipid in the simulations, Table 3.5 and Fig. 3.15.

\section{Water Mean Dipole Orientation}

While a DMPC lipid molecule has a net zero charge, there are localized regions of charge in the phosphate and choline regions of the headgroup. Water molecules can permeate deep into this polar lipid headgroup region and can shield the charge from 


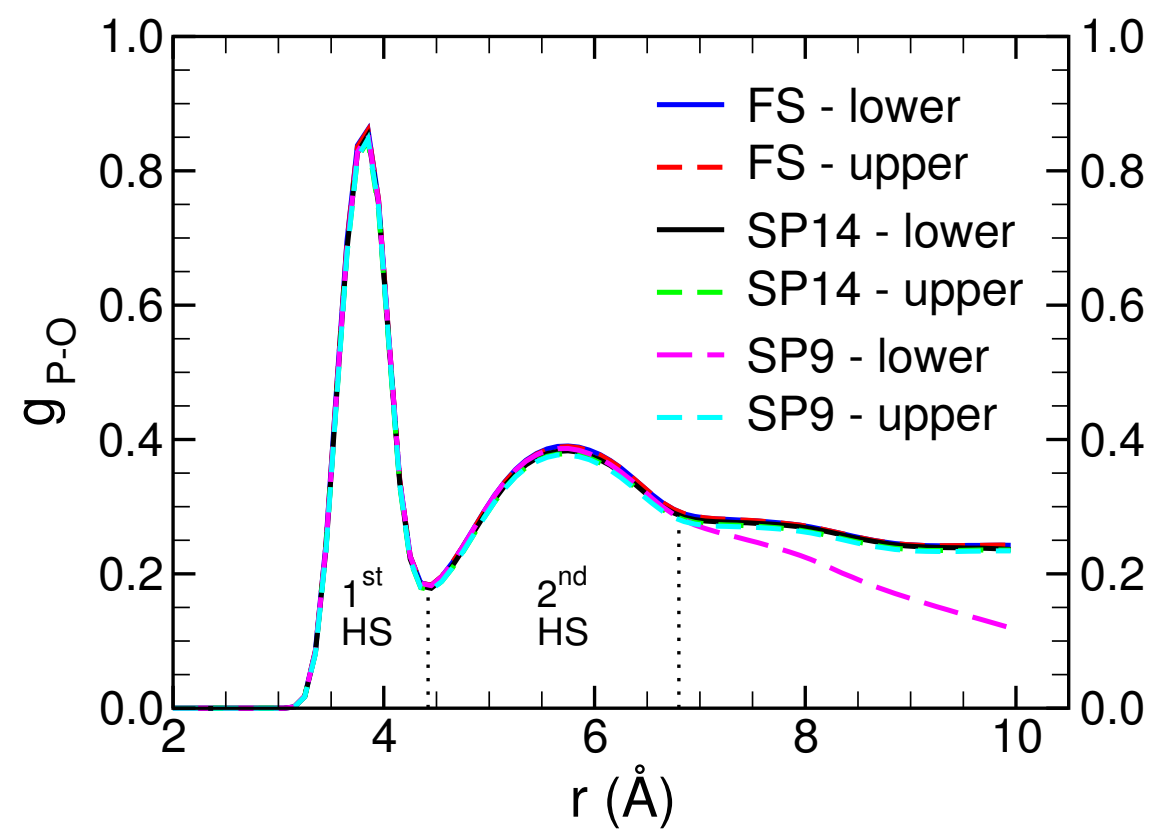

Figure 3.15: The water radial distribution function with respect to the phosphorus atom in each lipid. Little change exists when the silica substrate is introduced. The number of water molecules in each hydration shell are provided in Table 3.5.

neighboring lipids by reorienting their dipoles. To see if the introduction of the silica and small changes to the bilayer thickness had any effect on the water mean dipole orientation, the averaged dipole moment in slabs along $\hat{z}$ were determined, as shown in Fig. 3.16.

The dipole orientation is defined by,

Table 3.5: Water Molecules per Lipid

\begin{tabular}{lcccc}
\hline \hline & \multicolumn{3}{c}{ 1st Shell } & \multicolumn{2}{c}{ 2nd Shell } \\
& $0 \AA<z<4.4 \AA$ & $0 \AA<z<6.8 \AA$ \\
\hline Simulation & Upper & Lower & Upper & Lower \\
\hline FS & 5.3 & 5.3 & 13.0 & 13.0 \\
SP14 & 5.2 & 5.2 & 12.7 & 12.8 \\
SP9 & 5.2 & 5.2 & 12.7 & 12.9 \\
\hline
\end{tabular}



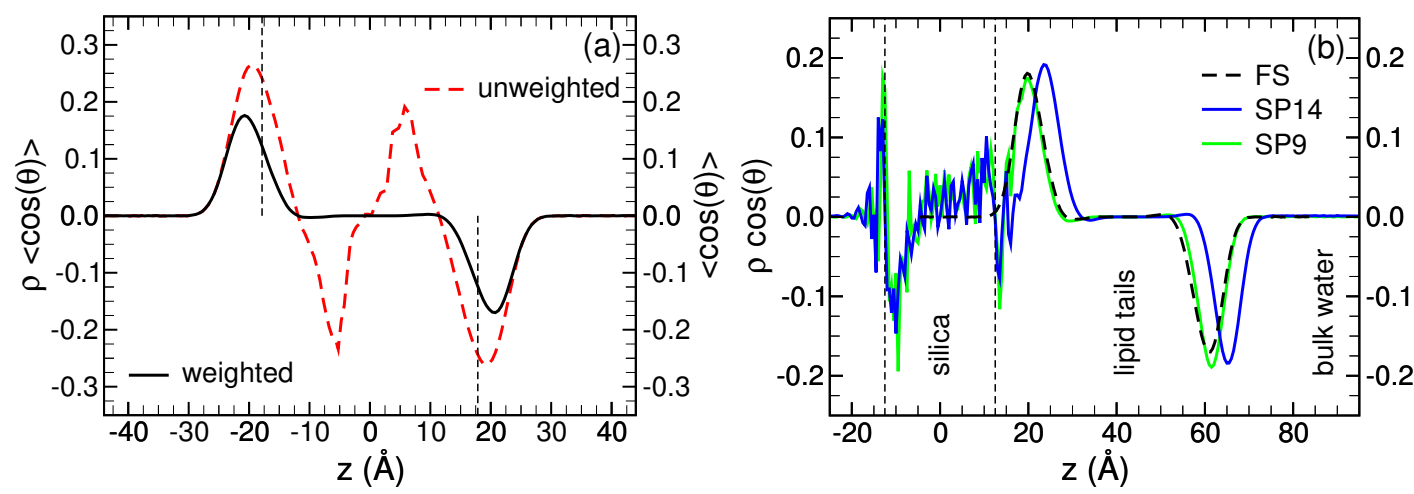

Figure 3.16: (a) The mean water dipole orientation profile for FS shown both with (black line) and without (red dashed) being weighted with the corresponding water mass density to demonstrate more clearly the overall influence of the water dipole. The location of the mean phosphorus atom for each leaflet has been drawn as vertical dashed lines and the center of the bilayer is located at $z=0 \AA$. (b) The weighted mean water dipole orientation profiles for the supported systems have been overlaid with that of the FS membrane to show there is little change due to the presence of the substrate. The vertical dashed lines show the location of each silica surface.

$$
\cos \theta=\frac{p_{z}}{|\vec{p}|}
$$

where $p_{z}$ is the component of the dipole vector, $\vec{p}$, along $\hat{z}$. The mean dipole orientation has a broad distribution in the interior of the membrane, $|z| \leq 10 \AA$, however, there are very few waters in this region. To show where significant dipole concentrations occur, the profiles are weighted with the corresponding water mass density value. The only change to the profile in the supported membrane systems is a slight increase in the peak maxima which is likely due to increasing water in the region due to the bilayer thickening. No significant changes are noticeable near the substrate surface. 


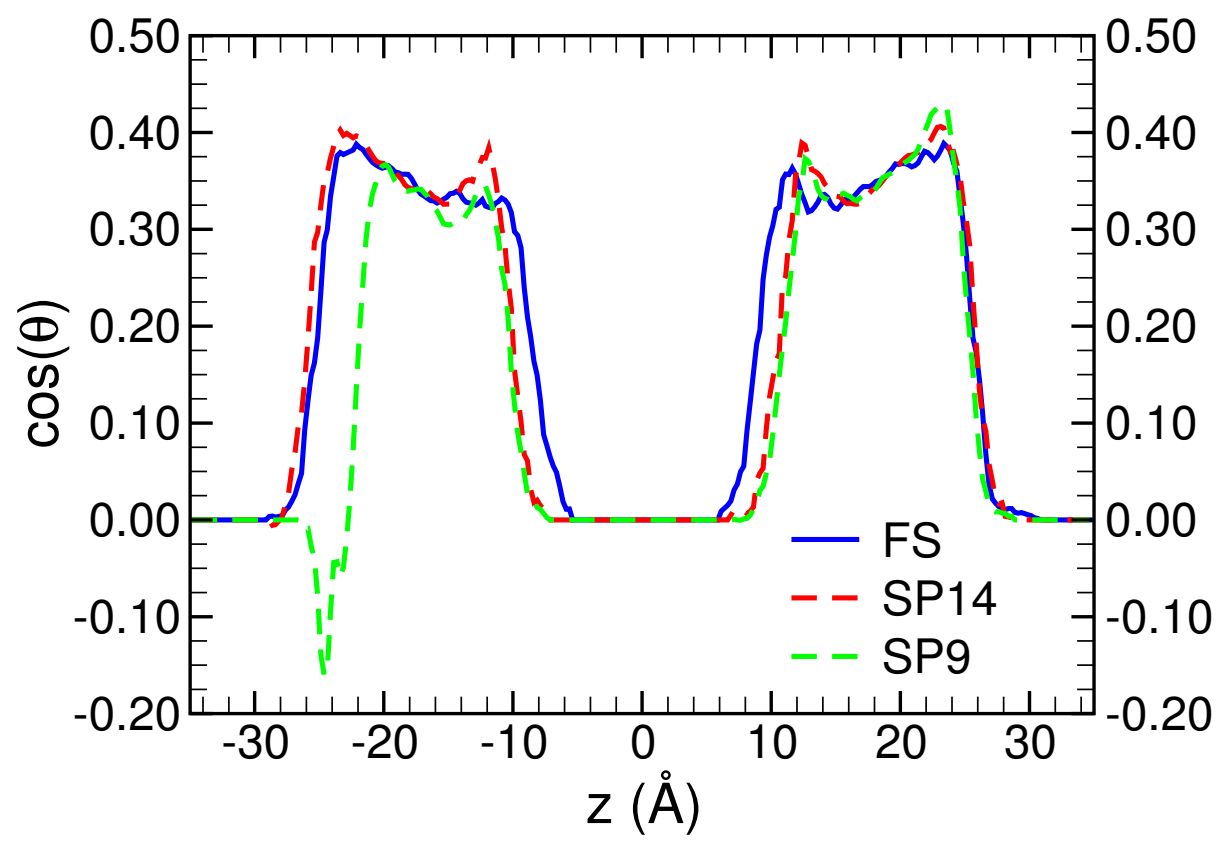

Figure 3.17: The mean orientation of the phosphorus to nitrogen, PN, vector in each lipid binned with respect to the $z$ coordinate values of the phosphorus atoms. FS and SP14 have similar profiles other than the slight widening due to the increased bilayer thickness. For SP9, the PN vector inverts for lipids near the silica substrate (large negative $z$ values).

\section{P-N Vector Orientation}

The mean orientation of the phosphorus to nitrogen vector in each lipid, called the PN dipole vector, has been calculated for $0.25 \AA$ wide slabs along $\hat{z}$ and have been binned with respect to the $z$ coordinate of the phosphorus atoms. The region of interest is roughly $z=( \pm) 18 \pm 3$ since that is where the phosphorus atoms are most likely to be located. When lipids in SP9 get near the silica substrate (large negative z values) the $\mathrm{PN}$ vector inverts. Other than this region, the PN vector value is relatively constant near $71^{\circ}$, see Fig. 3.17. 


\section{Mean Square Displacement and Diffusion Coefficients}

\section{Lipids}

A previous MD simulation similarly found no change to the mass density and water dipole profiles but did find a decrease in the lateral diffusion coefficient for the lipids in the leaflet closest to the substrate [57]. The system studied was a POPC lipid bilayer supported on silica with an HLT of $15.4 \AA$ in an NVT ensemble at 298 K. On the 10-25 ns timescale, the lipid headgroup center-of-mass lateral diffusion coefficients were $D=1.9 \times 10^{-8} \mathrm{~cm}^{2} / \mathrm{s}$ for the lower leaflet and $D=5.4 \times 10^{-8} \mathrm{~cm}^{2} / \mathrm{s}$ for the upper leaflet, a $65 \%$ decrease for the lower leaflet. On the $t \leq 10 \mathrm{~ns}$ time scale, an $18 \%$ decrease was reported for the lower leaflet.

In experiment, there are conflicting results as to whether this change exists. In fluorescence correlation spectroscopy experiments only a $5-10 \%$ reduction in the lower leaflet lipid lateral diffusion coefficient was found but this was within the margin of error [58]. NMR experiments at $328 \mathrm{~K}$ report a $50 \%$ reduction in the diffusion coefficient for the lower leaflet, $7.5 \times 10^{-8} \mathrm{~cm}^{2} / \mathrm{s}$, with respect to the upper leaflet, $1.4 \times 10^{-7} \mathrm{~cm}^{2} / \mathrm{s}[59]$.

For our supported system SP14, where $z^{\prime}=13.9 \AA$, both the silica and lower leaflet surfaces are nearly fully hydrated, as shown in the water mass density profiles in Fig. 3.14 and water radial distribution functions in Fig.3.15, which is why our results show little difference for the lateral MSDs with the introduction of the silica at this separation. When the water layer thickness is decreased further to $z^{\prime}=9.3 \AA$ in SP9, then the lipids begin to interact with the silica hydration layer which slows down the lipid in-plane motion. 


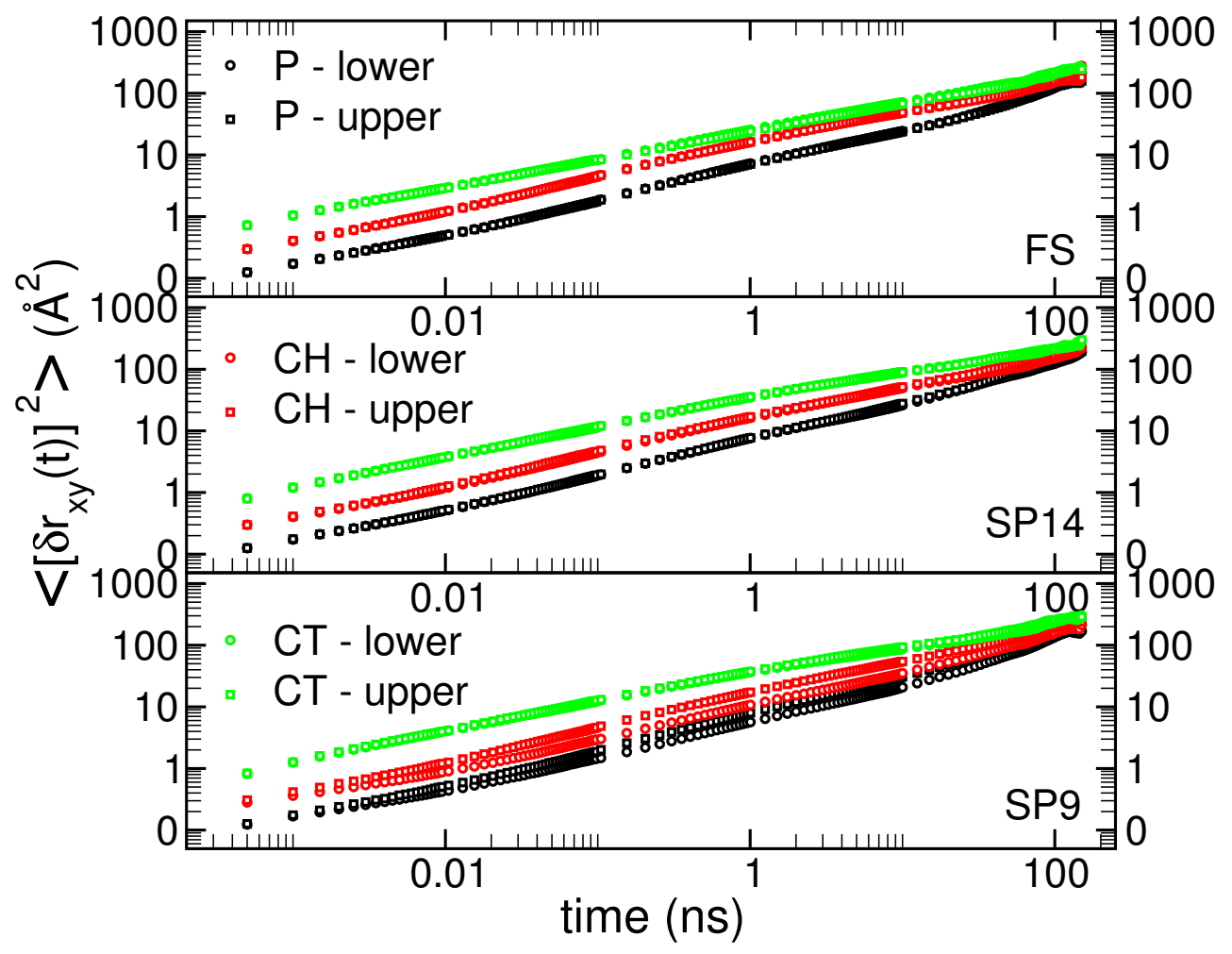

Figure 3.18: Lateral mean square displacement of the lipid phosphorus ( $\mathrm{P}$, black), headgroup carbon (CH, red), and tail carbon (CT, green) atoms is shown. The upper leaflet (boxes) and lower leaflet (circles) data overlay each other for the entire time range for FS and SP14. Additionally, there is no difference between the upper and lower leaflet for $\mathrm{CT}$ in the simulation with the bilayer closest to the substrate, SP9, but the MSD for $\mathrm{CH}$ and $\mathrm{P}$ atoms in the lower leaflet does show a decrease with respect to the upper leaflet.

To better understand the lateral motion of the lipid molecule, we have tracked three atoms in each lipid over the entire simulation: phosphorus $(\mathrm{P})$, a headgroup carbon $(\mathrm{CH})$, and the terminal or tail carbon $(\mathrm{CT})$, as shown in Fig. 3.2a. The results in Fig. 3.18 demonstrate that all three atoms have the same MSD for FS and SP14 when comparing the upper and lower leaflets. When the membrane was moved closer to the substrate in SP9, the MSDs for the headgroup atoms, $\mathrm{CH}$ and $\mathrm{P}$, show a splitting between the upper and lower leaflet, while the MSD of CT is unaffected. 
It is important to note that the MSDs of all the atoms should overlap when the lipid is undergoing free diffusion, which is clearly not the case here until $t>100 \mathrm{~ns}$.

When we compare the results between simulations, we see that for the $\mathrm{P}$ atoms in SP14 and SP9 (excluding the lower leaflet in SP9) the MSDs are slightly larger than in FS, as shown in Fig. 3.19. The increase may be due to the slight thickening of the lipid bilayer without the normal decrease in APL that usually accompanies it, which could provide the lipid more room to move. We do not see this behavior for the SP9 lower leaflet lipids because they are affected by the proximity of the silica and their interaction with the silica hydration waters. As a result their motion is slowed down. This reduction also occurs, but less dramatically, for SP14 for which there is a splitting between the lower and upper leaflet phosphorus MSD (Fig. 3.19b).

In our simulations, the lateral diffusion coefficients were determined from the slope of a linear fit to the lateral MSD data,

$$
D=\frac{1}{4} \frac{d}{d t}\left\langle\left(x-x_{0}\right)^{2}+\left(y-y_{0}\right)^{2}\right\rangle
$$

The diffusion coefficients from $2 \leq t \leq 10$ ns for the phosphorus atoms are provided in Table 3.6. For $t<2$ ns the phosphorus MSDs are very nonlinear and on longer time scales $(t>100 \mathrm{~ns})$ the data is difficult to fit because the window averaged statistics are of lower quality. A diffusion coefficient near $D=0.9 \times 10^{-7} \mathrm{~cm}^{2} / \mathrm{s}$ is inferred for the $t>50$ ns regime.

A previous MD simulation of a hydrated DMPC lipid bilayer determined the phosphorus lateral diffusion coefficient to be $1.3 \times 10^{-7} \mathrm{~cm}^{2} / \mathrm{s}$ in the $30 \leq t \leq 100 \mathrm{~ns}$ time regime but their simulation used a Langevin friction coefficient of $0.05 \mathrm{ps}^{-1}$ [14], while our simulations used $5 \mathrm{ps}^{-1}$. Another MD simulation with an identical friction 

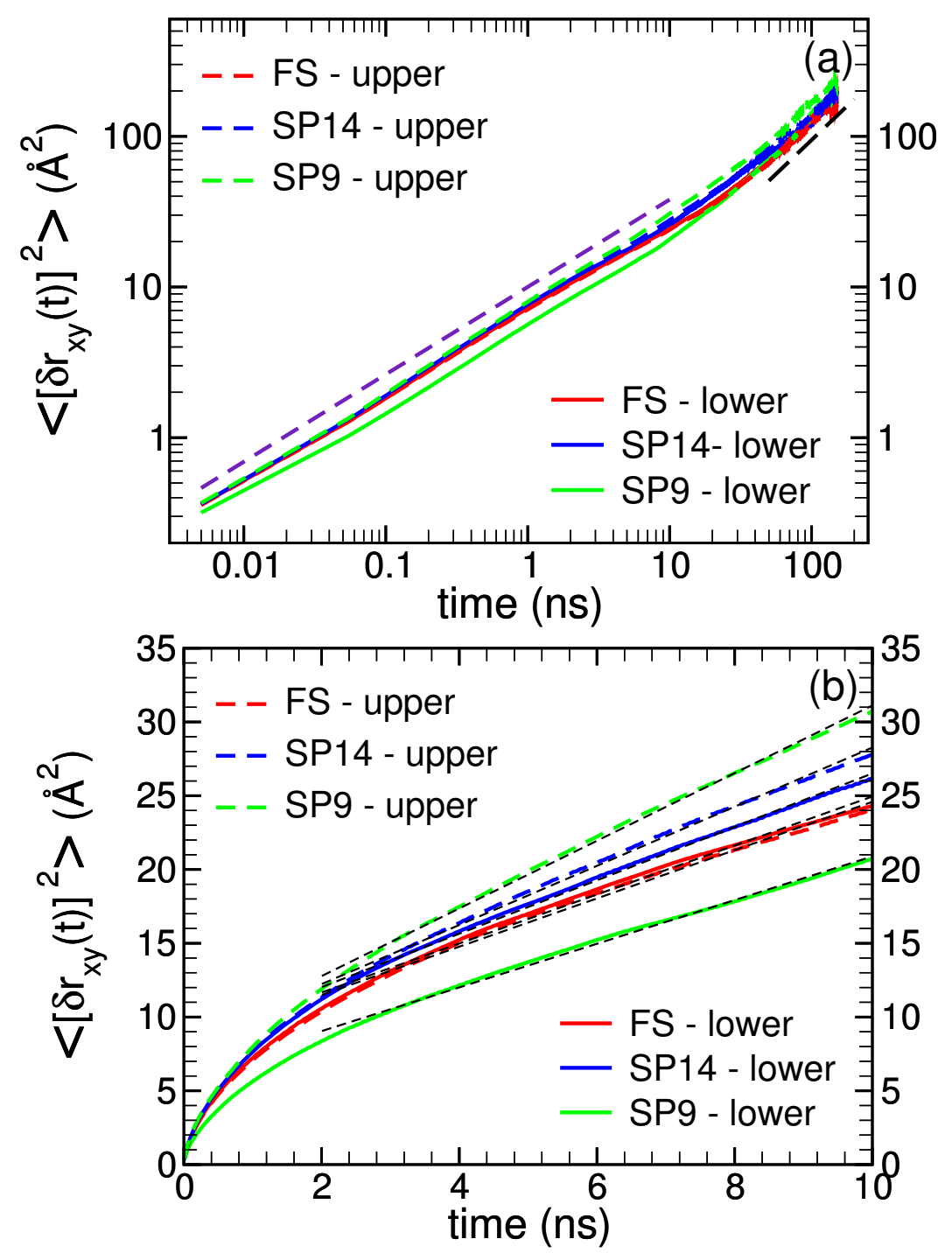

Figure 3.19: (a) Lateral mean square displacement of the lipid phosphorus atoms over the $150 \mathrm{~ns}$ simulations. Two lines have also been drawn as a reference. From $0.005 \leq t \leq 10 \mathrm{~ns}$, the line (purple dashed) corresponds to a diffusion coefficient of $0.58 \times 10^{-7} \mathrm{~cm}^{2} / \mathrm{s}$ and for $t>50 \mathrm{~ns}$ the line (black dashed) to $D=0.9 \times 10^{-7} \mathrm{~cm}^{2} / \mathrm{s}$. (b) A closer view from $0 \leq t \leq 10 \mathrm{~ns}$ on a linear scale displays a fit for each of the curves. Values from the fits are provided in Table 3.6. 
Table 3.6: Lipid Phosphorus Lateral Diffusion Coefficients

\begin{tabular}{lcc}
\multicolumn{3}{c}{ for $2 \leq t \leq 10 \mathrm{~ns}\left(\times 10^{-7} \mathrm{~cm}^{2} s^{-1}\right)$} \\
\hline \hline Simulation & Upper Leaflet & Lower Leaflet \\
\hline FS & 0.409 & 0.415 \\
SP14 & 0.501 & 0.453 \\
SP9 & 0.573 & 0.370 \\
\hline
\end{tabular}

coefficient to ours found a diffusion coefficient of $D=1.24 \times 10^{-7} \mathrm{~cm}^{2} / \mathrm{s}$ in the $1 \leq t \leq 2$ ns regime [56]. The FRAP experimental value is $0.69 \times 10^{-7} \mathrm{~cm}^{2} / \mathrm{s}$ [60].

The general trend is that the phosphorus lateral diffusion coefficient increases from FS to SP14 to SP9, excluding the SP9 lower leaflet which shows a $35 \%$ decrease due the lipid interactions with the silica hydration water on the $t<10$ ns time scale which is a larger decrease than what was reported in a previous MD simulation, which had an $18 \%$ decrease, on this time scale [57]. The decrease seen in our simulation is most likely due to the HLT being $1.1 \AA$ smaller in SP9 with respect to the prior simulation. Note also that the SP14 lower leaflet diffusion coefficient is also smaller with respect to the upper leaflet, likely for the same reason.

On the $10 \leq t \leq 50$ ns time scale, we do not see the $65 \%$ smaller $D$ value for the lower leaflet lipids that was reported previously [57]. The results for the lipid phosphorus lateral diffusion coefficients on this time scale are provided in Table 3.7. The same general trend is seen, with respect to the $2 \leq t \leq 10 \mathrm{~ns}$ time scale, when going from FS to SP14 to SP9 the $D$ values increase, except for the lower leaflet in SP9. Also, in the supported systems the lower leaflet diffusion coefficients are smaller, where the biggest difference again is with SP9, but here the lower leaflet $D$ is only $18 \%$ smaller than the upper leaflet. 
Table 3.7: Lipid Phosphorus Lateral Diffusion Coefficients

\begin{tabular}{lcc}
\multicolumn{3}{c}{ for $10 \leq t \leq 50 \mathrm{~ns}\left(\times 10^{-7} \mathrm{~cm}^{2} s^{-1}\right)$} \\
\hline \hline Simulation & Upper Leaflet & Lower Leaflet \\
\hline FS & 0.263 & 0.278 \\
SP14 & 0.343 & 0.320 \\
SP9 & 0.368 & 0.303 \\
\hline
\end{tabular}

\section{Water}

In QENS experiments, diffusion coefficients can be determined for different water layers by freezing out layers or by differing the amount of water introduced in a sample. In the MD simulations, we break up the water layers by defining boundaries based on the $z$ coordinates of the water molecules. Then the mean square displacement is determined in each layer only tracking the water molecules for as long as they stay in the water layer that they started in. For the free-standing system, the upper and lower leaflets are identical but for the supported systems the analysis is complicated by the fact that waters below the membrane are not in the same environment as the upper leaflet waters due their interaction with the silica substrate.

The QENS measurements were taken on the high flux backscattering spectrometer (HFBS) [61] at the NIST Center for Neutron Research and backscattering silicon spectrometer (BASIS) [62] at the Spallation Neutron Source at Oak Ridge National Laboratory. For these instruments, the energy resolutions are $\pm 1 \mu \mathrm{eV}$ (HFBS) and $\pm 3.5 \mu \mathrm{eV}$ (BASIS) corresponding to $4 \mathrm{~ns}$ and $1 \mathrm{~ns}$ time scales, respectively, which are the maximum time scale accessible in their experiments. In the MD simulations, while we have $150 \mathrm{~ns}$ long simulations, all of the water molecules (other than the water between the silica and the membrane) have exited their respective water layers before $t=4 \mathrm{~ns}$, but in many layers we still have a sufficient number of waters to reach 
Table 3.8: Water Layer Definitions I $(\AA)$

\begin{tabular}{lcccc}
\hline \hline Region & FS & SP14 & SP9 & Water Density $\left(\mathrm{g} / \mathrm{cm}^{3}\right)$ \\
\hline 1 & $28<z<45.35$ & $72.5<z<103$ & $68<z<103$ & $\rho \geq 1$ \\
2 & $18<z<28$ & $62.5<z<72.5$ & $58<z<68$ & $0.5<\rho<1$ \\
3 & $0<z<18$ & $44.5<z<62.5$ & $40<z<58$ & $\rho \leq 0.5$ \\
4 & $-18<z<0$ & $26.5<z<44.5$ & $22<z<40$ & $\rho \leq 0.5$ \\
5 & $-28<z<-18$ & $16.5<z<26.5$ & $12<z<22$ & $0.5<\rho<1$ \\
6 & $-45.35<z<-28$ & $12<z<16.5$ & - & $\rho \geq 1$ \\
\hline
\end{tabular}

the $t=1 \mathrm{~ns}$ time scale.

In our systems, the water molecules have been split into three layers on each leaflet: the inner water layer consists of water from the center of the membrane to roughly the mean phosphorus location, $\left|\left\langle z_{p}\right\rangle\right| \approx 18 \AA$; the middle region is from $\left\langle z_{p}\right\rangle$ to where the lipid mass density goes to zero, $|z| \approx 28 \AA$; and the outer water layer, $|z|>28 \AA$, where all the waters are entirely outside the membrane. These two boundaries also coincide with where the water mass density is about $50 \%$ of the bulk water mass density, $\rho_{\text {water }} \approx 0.5 \mathrm{~g} / \mathrm{cm}^{3}$ at $|z|=18 \AA$, and near $100 \%$ the bulk value, $\rho_{\text {water }} \approx 1.0 \mathrm{~g} / \mathrm{cm}^{3}$ at $|z|=28 \AA$.

For the supported systems, the lower leaflet outer water (layer 6 in Fig. 3.20) is either the silica hydration water, for SP14, or missing, for SP9, because the membrane is in such close proximity to the substrate. The center of the supported bilayers have a $z$ coordinate of $44.5 \AA$ and $40 \AA$ for SP14 and SP9, respectively. For FS, the center is at $z=0 \AA$. Also, for FS, regions 1 and 6 were combined into a single region since they are in contact across the periodic boundary. The $z$ boundaries for each simulation are listed in Table 3.8 and displayed in Fig. 3.20.

One complication is that even within a water layer the water diffusion coefficients are $z$ dependent as the water molecules deeper inside the membrane diffuse slower 

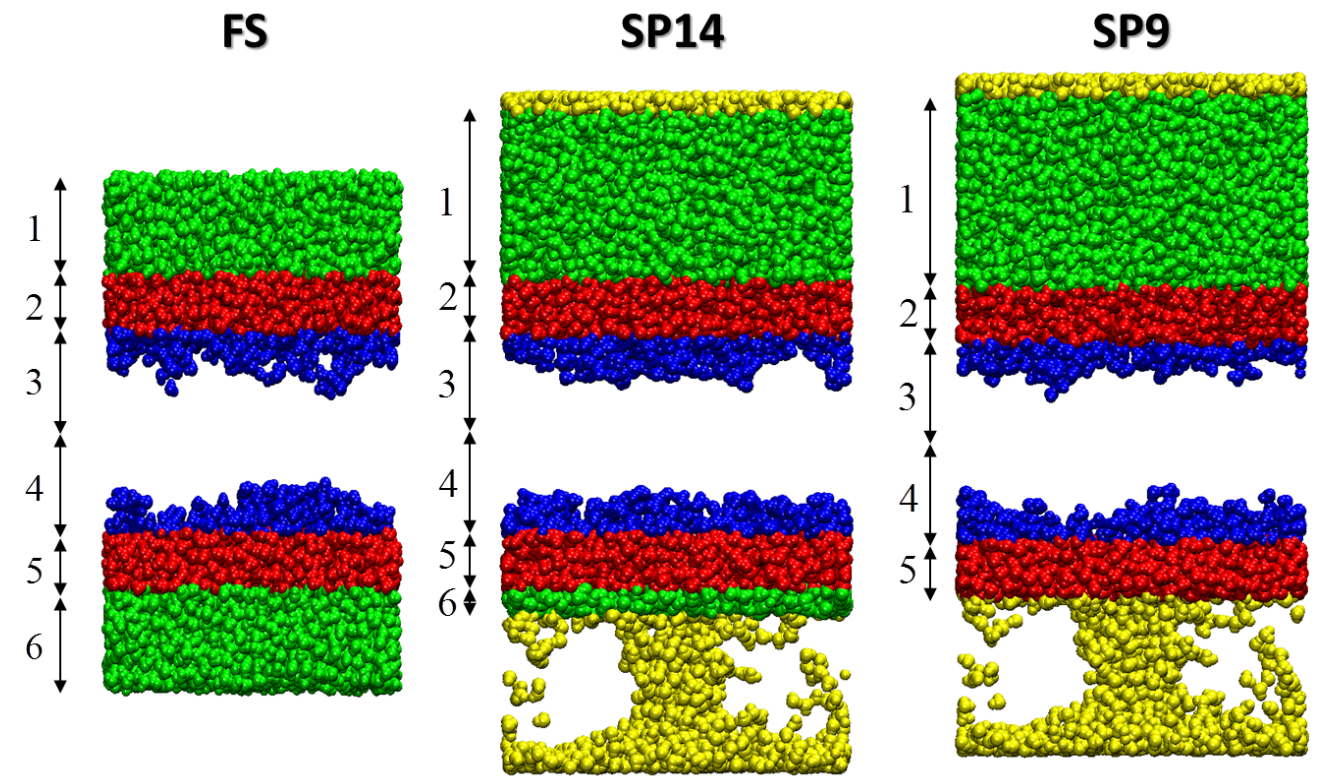

Figure 3.20: Snapshot of water molecules in all three systems colored by their water layers. The boundaries are defined in Table 3.8. The water molecules (colored yellow) in the silica pore and the hydration water on the lower surface of the silica were not included in any water layer when determining the MSD.

than those that are further from the bilayer center. Also, the waters near the top of a layer can more easily exit their water layer leaving only the slower moving waters to be included in the MSD calculations. In some cases, more than $90 \%$ of waters leave within a few hundred picoseconds. For each layer, the residency of the waters in the layer, defined as the number, $N$, of waters in a layer divided by the initial number, $N_{0}$, in the layer have also been determined.

The time evolution of $N / N_{0}$ and the MSDs for each water layer are shown in Fig. 3.21 and the corresponding lateral diffusion coefficients are listed in Table 3.9. For water layers $1,2,5$, and 6 , the linear fit of the MSD included all the data up to $t=1 \mathrm{~ns}$. For water layers 3 and 4 , the MSD results are very noisy and in some cases decrease at longer time scales. The fit to the MSD data for water layers 3 and 4 only 

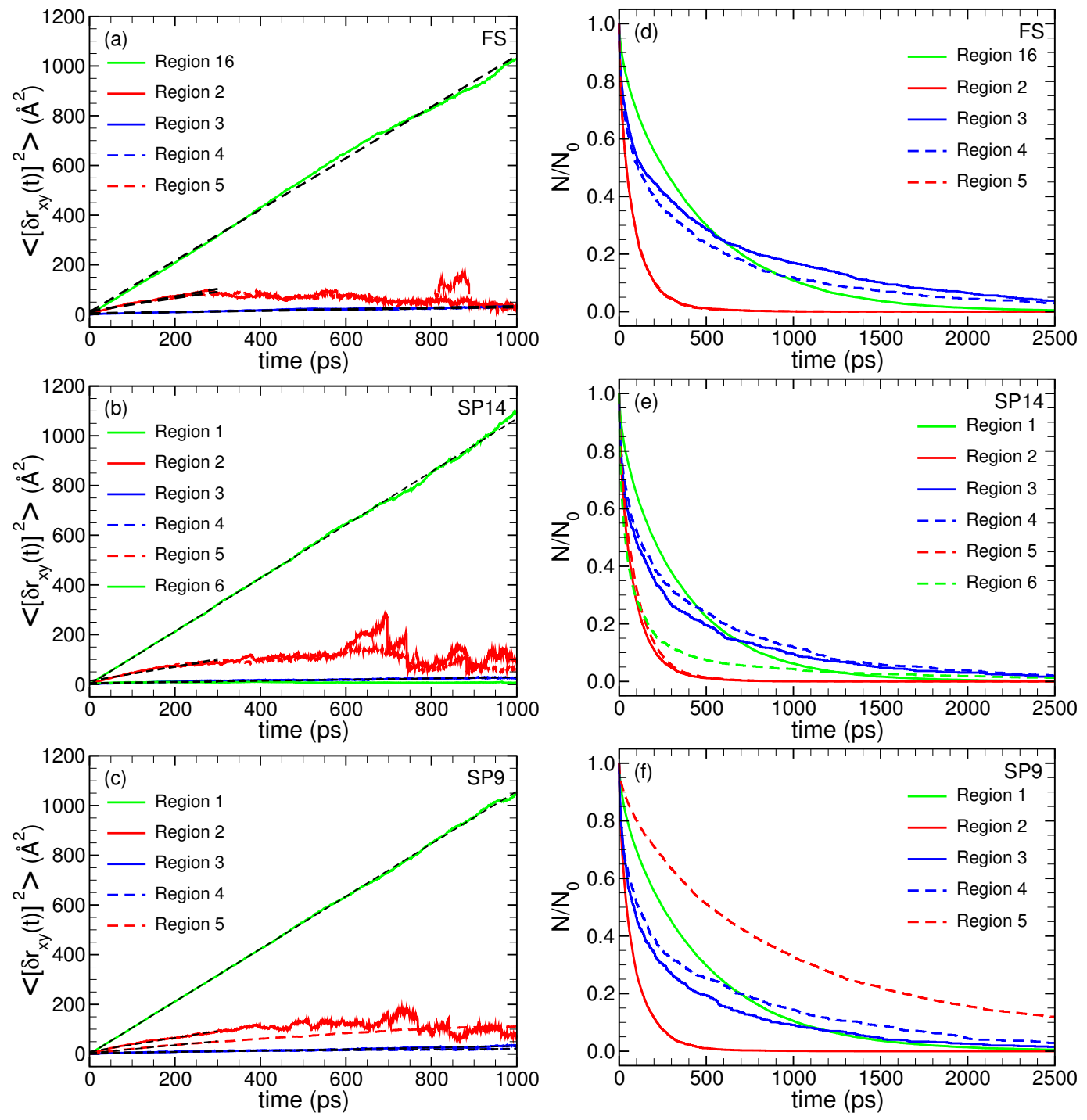

Figure 3.21: (a-c) Lateral mean square displacement of the water molecules with the hydration layers defined in Table 3.8. Linear fits to the MSDs returned the diffusion coefficient values listed in Table 3.9. (d-f) Residency of water molecules as a function of time. 
Table 3.9: Water Lateral Diffusion Coefficients $\left(\times 10^{-5} \mathrm{~cm}^{2} / \mathrm{s}\right)$

\begin{tabular}{lccc}
\hline \hline Region & FS & SP14 & SP9 \\
\hline 1 & 2.58 & 2.68 & 2.65 \\
2 & 0.78 & 0.70 & 0.70 \\
3 & 0.073 & 0.055 & 0.075 \\
4 & 0.060 & 0.063 & 0.040 \\
5 & 0.63 & 0.75 & 0.40 \\
6 & $*$ & 0.033 & - \\
\hline
\end{tabular}

included the $t<0.3 \mathrm{~ns}$ regime.

The water lateral diffusion coefficients cover multiple orders of magnitude as the outer layers are all slightly larger than the experimental value for bulk water, $D_{\exp }=$ $2.33 \times 10^{-5} \mathrm{~cm}^{2} / \mathrm{s}[63,64]$. Previous MD simulations with the same damping coefficient of $5 \mathrm{ps}^{-1}$ had a bulk water lateral diffusion coefficient of $D=2.65 \times 10^{-5} \mathrm{~cm}^{2} / \mathrm{s}[15,65]$.

A previous MD simulation [56] of a free-standing bilayer with the same temperature controls divided the water into layers, in a similar manner to our definitions, but found a reduced bulk water diffusion coefficient of $D \approx 1.45 \times 10^{-5} \mathrm{~cm}^{2} / \mathrm{s}$. The smaller value is partially due to the bulk water being broken up into two layers which allowed the faster moving water molecules to escape their respective layers more quickly and therefore they were not included in the longer time MSD.

The paper also reported $D \approx 4.95 \times 10^{-6} \mathrm{~cm}^{2} / \mathrm{s}$ for regions 2 and 5 , and $D \approx$ $1.68 \times 10^{-6} \mathrm{~cm}^{2} / \mathrm{s}$ for regions 3 and 4 . The smaller values, compared to our results, for layers $1,2,5$, and 6 are likely due to the shorter time scale they studied, $20 \leq$ $t \leq 100$ ps. The larger values for layers 3 and 4 may be due to the fact that their diffusion coefficients are three dimensional, not lateral, and the contribution along the $z$ direction for these regions may dominate the calculated $D$.

For the middle water layers ( 2 and 5 ), in FS and SP14, $D \approx 0.70 \times 10^{-5} \mathrm{~cm}^{2} / \mathrm{s}$, 
Table 3.10: Water Layer Definitions II $(\AA)$

\begin{tabular}{lccc}
\hline \hline Region & FS & SP14 & SP9 \\
\hline 23 & $0<z<28$ & $44.5<z<72.5$ & $40<z<68$ \\
45 & $-28<z<0$ & $16.5<z<44.5$ & $12<z<40$ \\
\hline $23+$ & $0<z<32.5$ & $44.5<z<77$ & - \\
456 & $-32.5<z<0$ & $12<z<44.5$ & - \\
\hline 56 & - & $12<z<26.5$ & - \\
\hline
\end{tabular}

while in SP9, $D$ is $50 \%$ smaller. This is due the fact that the water in layer 5, in SP9, hydrates both the lipids and the silica substrate. In QENS experiments at $265 \mathrm{~K}$, where the bulk water has been frozen out, a diffusion coefficient of $D=$ $0.61 \times 10^{-5} \mathrm{~cm}^{2} / \mathrm{s}$ was determined for the remaining mobile water [13], which is very close to our results for water layers 2 and 5 . The inner water layers, 3 and 4 , show a further reduction with values near $D=0.065 \times 10^{-5} \mathrm{~cm}^{2} / \mathrm{s}$, except again, for SP9, where $D$ is reduced by an additional $50 \%$.

Another method we employed, to estimate $D$, used thicker water layers to show more clearly the differences between the dynamics of the waters above and below the membrane. Water layers 2 and 3 were combined into a single water layer denoted as 23, water layers 4 and 5 became 45, etc. The definitions are shown in Table 3.10 and resulting MSDs in Fig. 3.22. These MSDs exhibit very different behavior than with the previous definitions. First, because the water layer thicknesses increased, residency $\left(N / N_{0}\right)$ increased. Additionally, for the supported bilayers, the MSDs for all water layers located below the membrane can be fit up to $t=1 \mathrm{~ns}$ but the water layers on the upper leaflet do not display a linear regime until $t>200 \mathrm{ps}$.

The MSDs for the upper leaflet water display very similar behavior to the freestanding membrane but the water trapped between the silica and membrane have linear MSDs for the entire time window. The water molecules trapped between 

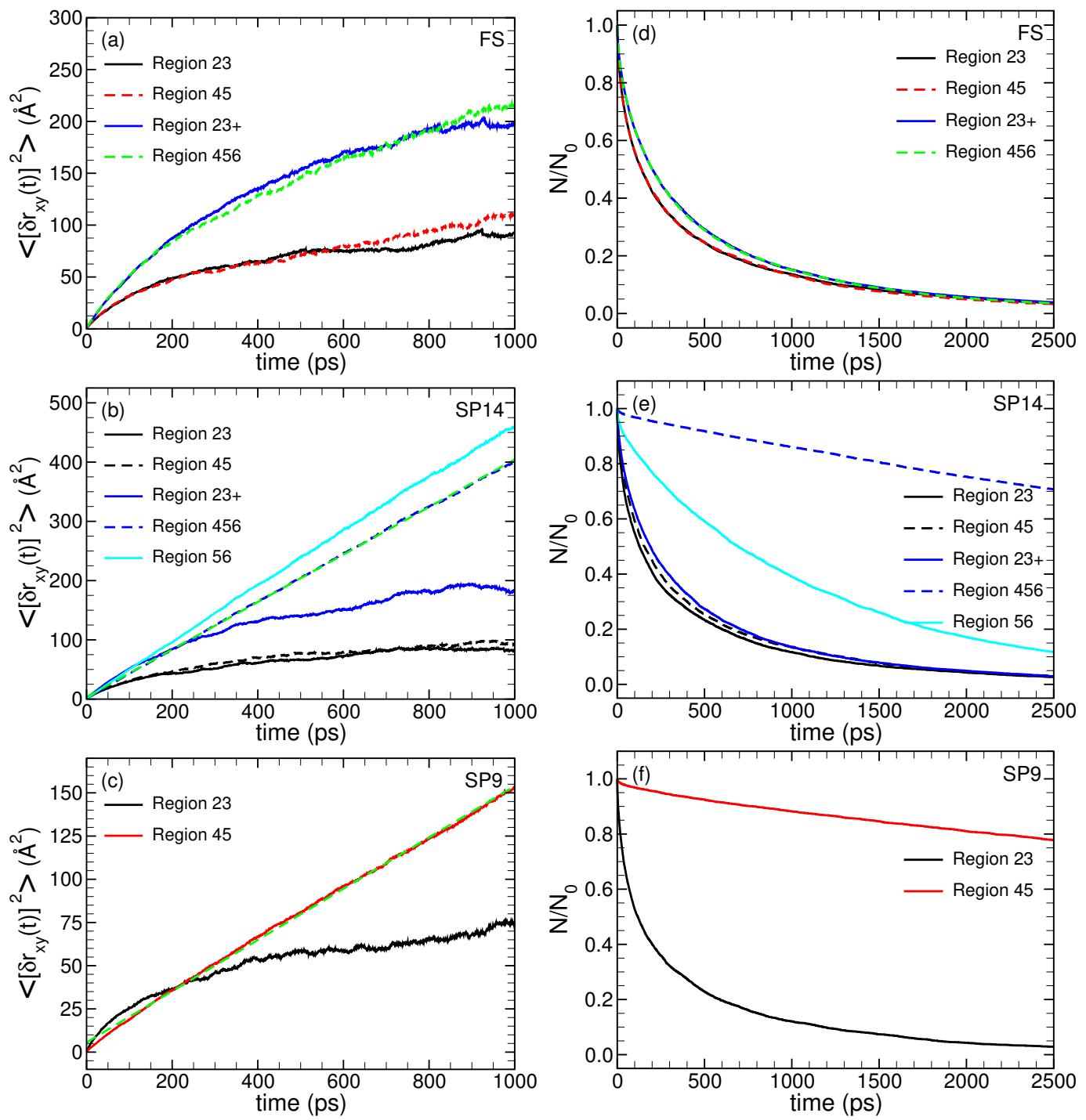

Figure 3.22: (a-c) Lateral mean square displacement of the water oxygen atoms with the water layers defined in Table 3.10. All water molecules between the membrane and the silica (water layer 456 for SP14 and layer 45 in SP9) are shown with linear fits (green dashed lines) in (b) for layer 456 in SP14 and (c) for layer 45 in SP9 with lateral water diffusion coefficients of $D=0.10 \times 10^{-5} \mathrm{~cm}^{2} / \mathrm{s}$ and $D=0.037 \times 10^{-5} \mathrm{~cm}^{2} / \mathrm{s}$, respectively. (d-f) Residency $\left(N / N_{0}\right)$ for water molecules in each water layer as a function of time. 
the silica and bilayer have high residency values since the water can only escape by entering the silica or through the membrane. These confined water layers, 456 (SP14) and 45 (SP9), had lateral diffusion coefficients of $D=0.10 \times 10^{-5} \mathrm{~cm}^{2} / \mathrm{s}$ and $D=0.037 \times 10^{-5} \mathrm{~cm}^{2} / \mathrm{s}$ for SP14 and SP9, respectively.

\subsection{Conclusion}

Even at the smallest water layer distance, between the silica and membrane, the lipids retained full hydration and there were few differences between the free-standing and supported systems in the mass density and water dipole orientation profiles, as well as the acyl chain order parameter. The only significant change to the PN vector orientation was seen when lipids were extremely close to the silica, indicating that a further reduction of the water layer between the substrate and the membrane may have more dramatic effects as the inversion of the PN vector would flip the electrostatic potential at the membrane surface [48].

At small separation distances, between the membrane and silica, water molecules were shared by both surfaces which led to smaller diffusion coefficients for the water and also for the lower leaflet lipids. The water molecules that hydrated the lipid headgroups were found to have lateral diffusion coefficient values very similar to QENS experiments [13] where the bulk water was frozen out of the measurement. Additionally, the water that was between the substrate and the membrane experienced a reduction in the water lateral diffusion coefficient that appeared to be dependent on the "trapped" water layer thickness. For SP9, where the membrane was closest to the silica, the lateral diffusion coefficient was reduced by more than an order of 
magnitude, with respect to a similar water layer in the upper leaflet.

The decrease in the lipid phosphorus lateral diffusion coefficient for the lower leaflet reported here for the $2 \leq t \leq 10$ ns time range was $35 \%$, a larger decrease than what was reported previously in a supported POPC bilayer MD simulation, which had an $18 \%$ decrease [57]. The paper additionally reported a $65 \%$ decrease for the lower leaflet lipid lateral diffusion coefficient on the $10 \leq t \leq 25$ ns time scale. In our simulations, we also determined $D$ on the $10 \leq t \leq 50$ ns time scale but only saw an $18 \%$ reduction for the lower leaflet phosphorus lateral diffusion coefficient with respect to the upper leaflet. Experimental results are ambiguous as to whether the reduced diffusion coefficient exists $[58,59]$.

This research project studied the relatively simple case where a bilayer of net zero charge lipids, hydrated with pure water, was mounted on to a smooth silica surface. Results showed that the lipid bilayer thickness did increase as the bilayer was moved closer to the substrate and the surface tension on the hydrated bilayer also increased. This potentially could lead to a reduction of the APL in the lower leaflet, increase the thickness of the bilayer further, and trigger a transition to the gel phase. Further investigation is needed and there are many ways that complexity could be added to future simulations: adding ions to the water, charged lipids with counter ions, introducing a membrane protein, modifying the silica surface roughness, including explicit hydroxyls and silanols on the silica surface, altering the in-plane simulation box size which would change the APL, or lowering the hydration level of the membrane. 


\section{Bibliography}

[1] C. J. Ferris, K. G. Gilmore, G. G. Wallace, and M. In het Panhuis. Biofabrication: an overview of the approaches used for printing of living cells. Appl. Microbiol. Biotechnol., 97(10):4243-58, 2013.

[2] S. Tasoglu and U. Demirci. Bioprinting for stem cell research. Trends Biotechnol., $31(1): 10-9,2013$.

[3] F.P.W. Melchels, M.A.N. Domingos, T.J. Klein, J. Malda, P.J. Bartolo, and D.W. Hutmacher. Additive manufacturing of tissues and organs. Progress in Polymer Science, 37(8):1079-1104, 2012.

[4] Aleksander Skardal and Anthony Atala. Biomaterials for integration with 3-d bioprinting. Annals of biomedical engineering, 43(3):730-46, 2015.

[5] K. Jakab, A. Neagu, V. Mironov, R. R. Markwald, and G. Forgacs. Engineering biological structures of prescribed shape using self-assembling multicellular systems. Proceedings of the National Academy of Sciences of the United States of America, 101(9):2864-2869, 2004. 
[6] K. Jakab, C. Norotte, B. Damon, F. Marga, A. Neagu, C. L. Besch-Williford, A. Kachurin, K. H. Church, H. Park, V. Mironov, R. R. Markwald, G. VunjakNovakovic, and G. Forgacs. Tissue engineering by self-assembly of cells printed into topologically defined structures. Tissue Eng.: Part A, 14(3):413-421, 2008.

[7] K. Jakab, C. Norotte, F. Marga, K. Murphy, G. Vunjak-Novakovic, and G. Forgacs. Tissue engineering by self-assembly and bio-printing of living cells. Biofabrication, 2(2):1, 2010.

[8] Elijah Flenner, Lorant Janosi, Bogdan Barz, Adrian Neagu, Gabor Forgacs, and Ioan Kosztin. Kinetic monte carlo and cellular particle dynamics simulations of multicellular systems. Phys. Rev. E, 85(3):031907, 2012.

[9] I. Kosztin, G. Vunjak-Novakovic, and G. Forgacs. Colloquium: Modeling the dynamics of multicellular systems: Application to tissue engineering. Rev. Mod. Phys., 84(4):1791-1805, 2012.

[10] M. McCune, A. Shafiee, G. Forgacs, and I. Kosztin. Predictive modeling of post bioprinting structure formation. Soft Matter, 10(11):1790-1800, 2014.

[11] A. Shafiee, M. McCune, G. Forgacs, and I. Kosztin. Post-deposition bioink selfassembly: a quantitative study. Biofabrication, 7(4):045005, 2015.

[12] M. Bai, A. Miskowiec, F. Y. Hansen, H. Taub, T. Jenkins, M. Tyagi, S. O. Diallo, E. Mamontov, K. W. Herwig, and S. K. Wang. Study of water diffusion on single-supported bilayer lipid membranes by quasielastic neutron scattering. EPL (Europhysics Letters), 98(4):48006, 2012. 
[13] A. Miskowiec, Z. N. Buck, M. C. Brown, H. Kaiser, F. Y. Hansen, G. M. King, H. Taub, R. Jiji, J. W. Cooley, M. Tyagi, S. O. Diallo, E. Mamontov, and K. W. Herwig. On the freezing behavior and diffusion of water in proximity to singlesupported zwitterionic and anionic bilayer lipid membranes. EPL (Europhysics Letters), 107(2):28008, 2014.

[14] E. Flenner, J. Das, M. C. Rheinstadter, and I. Kosztin. Subdiffusion and lateral diffusion coefficient of lipid atoms and molecules in phospholipid bilayers. Physical Review E - Statistical, Nonlinear, and Soft Matter Physics, 79(1), 2009.

[15] Jhuma Das, Elijah Flenner, and Ioan Kosztin. Anomalous diffusion of water molecules in hydrated lipid bilayers. The Journal of Chemical Physics, 139:065102, 2013.

[16] R. Gordon, N. S. Goel, M. S. Steinberg, and L. L. Wiseman. A rheological mechanism sufficient to explain the kinetics of cell sorting. J. Theor. Biol., 37(1):43-73, 1972.

[17] José C. M. Mombach, James A. Glazier, Richard C. Raphael, and Mark Zajac. Quantitative comparison between differential adhesion models and cell sorting in the presence and absence of fluctuations. Phys. Rev. Lett., 75:2244-2247, 1995.

[18] D. A. Beysens, G. Forgacs, and J. A. Glazier. Cell sorting is analogous to phase ordering in fluids. Proc. Natl. Acad. Sci. U. S. A., 97(17):9467-9471, 2000.

[19] Steve Plimpton. Fast parallel algorithms for short-range molecular dynamics. $J$. Comput. Phys., 117(1):1-19, 1995. 
[20] J. Frenkel. Viscous flow of crystalline bodies under the action of surface tension. J. Physics, 9:385-391, 1945.

[21] J. D. Eshelby. Trans. AIMME, 185:806, 1949.

[22] Wolfram Research, Inc. Mathematica, Version 9.0, Champaign, IL, 2012.

[23] William Humphrey, Andrew Dalke, and Klaus Schulten. VMD - Visual Molecular Dynamics. Journal of Molecular Graphics, 14:33-38, 1996.

[24] J. C. Phillips, R. Braun, W. Wang, J. Gumbart, E. Tajkhorshid, E. Villa, C. Chipot, R. D. Skeel, L. Kale, and K. Schulten. Scalable molecular dynamics with namd. Journal of Computational Chemistry, 26:1781-1802, 2005.

[25] N. Kucerka, Y. Liu, N. Chu, H. I. Petrache, S. Tristram-Nagle, and J. F. Nagle. Structure of fully hydrated fluid phase dmpc and dlpc lipid bilayers using xray scattering from oriented multilamellar arrays and from unilamellar vesicles. Biophysical Journal, 88(4):2626-2637, 2005.

[26] J. F. Nagle, R. Zhang, S. Tristram-Nagle, W. Sun, H. I. Petrache, and R. M. Suter. X-ray structure determination of fully hydrated 1 alpha phase dipalmitoylphosphatidylcholine bilayers. Biophysical Journal, 70(3):1419-1431, 1996.

[27] J. M. Zanotti, M. C. Bellissent-Funel, and J. Parello. Hydration-coupled dynamics in proteins studied by neutron scattering and nmr: the case of the typical ef-hand calcium-binding parvalbumin. Biophysical Journal, 76(5):2390-2411, 1999.

[28] J. B. Klauda, R. M. Venable, J. A. Freites, J. W. O'Connor, D. J. Tobias, C. Mondragon-Ramirez, I. Vorobyov, A. D. MacKerell Jr, and R. W. Pastor. 
Update of the charmm all-atom additive force field for lipids: Validation on six lipid types. Journal of Physical Chemistry B, 114(23):7830-7843, 2010.

[29] E. R. Cruz-Chu, A. Aksimentiev, and K. Schulten. Water-silica force field for simulating nanodevices. Journal of Physical Chemistry B, 110(43):21497-21508, 2006.

[30] Tom Darden, Darrin York, and Lee Pedersen. Particle mesh ewald: An nlog(n) method for ewald sums in large systems. The Journal of Chemical Physics, 98(12):10089-10092, 1993.

[31] Jeffrey R. Comer, David B. Wells, and Aleksei Aksimentiev. Case Study: Modeling Nanopores for Sequencing DNA, 2010.

[32] B. W. H. van Beest, G. J. Kramer, and R. A. van Santen. Force fields for silicas and aluminophosphates based on ab initio calculations. Physical Review Letters, 64(16):1955-1958, 1990.

[33] K. Vollmayr, W. Kob, and K. Binder. Cooling-rate effects in amorphous silica: A computer-simulation study. Phys Rev B Condens Matter, 54(22):15808-15827, 1996.

[34] D. B. Wells, V. Abramkina, and A. Aksimentiev. Exploring transmembrane transport through alpha-hemolysin with grid-steered molecular dynamics. $J$ Chem Phys, 127(12):125101, 2007.

[35] Gottfried Otting. Nmr studies of water bound to biological molecules. Progress in Nuclear Magnetic Resonance Spectroscopy, 31(2):259 - 285, 1997. 
[36] Jeannine Milhaud. New insights into waterphospholipid model membrane interactions. Biochimica et Biophysica Acta (BBA) - Biomembranes, 1663(12):19 $51,2004$.

[37] M. Hishida and K. Tanaka. Long-range hydration effect of lipid membrane studied by terahertz time-domain spectroscopy. Phys. Rev. Lett., 106:158102, Apr 2011.

[38] Wei Zhao, David E. Moilanen, Emily E. Fenn, and Michael D. Fayer. Water at the surfaces of aligned phospholipid multibilayer model membranes probed with ultrafast vibrational spectroscopy. Journal of the American Chemical Society, 130(42):13927-13937, 2008.

[39] S. Konig, E. Sackmann, D. Richter, R. Zorn, C. Carlile, and T. M. Bayerl. Molecular dynamics of water in oriented dppc multilayers studied by quasielastic neutron scattering and deuteriumnuclear magnetic resonance relaxation. The Journal of Chemical Physics, 100(4):3307-3316, 1994.

[40] Sundaram Balasubramanian, Subrata Pal, and Biman Bagchi. Evidence for bound and free water species in the hydration shell of an aqueous micelle. arXiv preprint physics/0212097, 2002.

[41] Michael R. Harpham, Branka M. Ladanyi, Nancy E. Levinger, and Kenneth W. Herwig. Water motion in reverse micelles studied by quasielastic neutron scattering and molecular dynamics simulations. The Journal of Chemical Physics, 121(16):7855-7868, 2004. 
[42] Zhancheng Zhang and Max L. Berkowitz. Orientational dynamics of water in phospholipid bilayers with different hydration levels. The Journal of Physical Chemistry B, 113(21):7676-7680, 2009.

[43] Shreyas Y. Bhide and Max L. Berkowitz. Structure and dynamics of water at the interface with phospholipid bilayers. The Journal of Chemical Physics, 123(22), 2005.

[44] A. K. Ronnest, G. H. Peters, F. Y. Hansen, H. Taub, and A. Miskowiec. Structure and dynamics of water and lipid molecules in charged anionic dmpg lipid bilayer membranes. The Journal of Chemical Physics, 144(14), 2016.

[45] A. Miskowiec, M. Bai, H. Taub, and F. Y. Hansen. Studies of the temperaturedependent structure of dmpc bilayer lipid membranes by atomic force microscopy. Bull. Am. Phys. Soc., 55, 2010. March Meeting of the American Physical Society, Portland, OR, 15-19 March 2010.

[46] Pieter R Cullis and Michael J Hope. Physical properties and functional roles of lipids in membranes. New Comprehensive Biochemistry, 20:1-41, 1991.

[47] B. A. Lewis and D. M. Engelman. Lipid bilayer thickness varies linearly with acyl chain length in fluid phosphatidylcholine vesicles. J Mol Biol, 166(2):211-7, 1983.

[48] E. A. Golovina, A. Golovin, F. A. Hoekstra, and R. Faller. Water replacement hypothesis in atomic details: effect of trehalose on the structure of single dehydrated popc bilayers. Langmuir, 26(13):11118-26, 2010. 
[49] S. Leekumjorn and A. K. Sum. Molecular dynamics study on the stabilization of dehydrated lipid bilayers with glucose and trehalose. J Phys Chem B, 112(34):10732-40, 2008.

[50] Dhaval A. Doshi, Andrew M. Dattelbaum, Erik B. Watkins, C. Jeffrey Brinker, Basil I. Swanson, Andrew P. Shreve, Atul N. Parikh, and Jaroslaw Majewski. Neutron reflectivity study of lipid membranes assembled on ordered nanocomposite and nanoporous silica thin films. Langmuir, 21(7):2865-2870, 2005.

[51] Justin Gullingsrud and Klaus Schulten. Lipid bilayer pressure profiles and mechanosensitive channel gating. Biophysical Journal, 86(6):3496-3509, 2004.

[52] Richard M. Venable, Frank L.H. Brown, and Richard W. Pastor. Mechanical properties of lipid bilayers from molecular dynamics simulation. Chemistry and Physics of Lipids, 192:60 - 74, 2015. \{ORNL\} workshop on Biomembranes.

[53] Scott E. Feller and Richard W. Pastor. Constant surface tension simulations of lipid bilayers: The sensitivity of surface areas and compressibilities. The Journal of chemical physics, 111(3):1281-1287, 1999.

[54] Torben Broemstrup and Nathalie Reuter. Molecular dynamics simulations of mixed acidic/zwitterionic phospholipid bilayers. Biophysical Journal, 99(3):825833, 2010.

[55] C. Faure, L. Bonakdar, and E. J. Dufourc. Determination of dmpc hydration in the 1 (alpha) and 1 (beta') phases by $2 \mathrm{~h}$ solid state $\mathrm{nmr}$ of d2o. FEBS Lett, 405(3):263-6, 1997. 
[56] F. Y. Hansen, G. H. Peters, H. Taub, and A. Miskowiec. Diffusion of water and selected atoms in dmpc lipid bilayer membranes. Journal of Chemical Physics, $137(20), 2012$.

[57] M. Roark and S. E. Feller. Structure and dynamics of a fluid phase bilayer on a solid support as observed by a molecular dynamics computer simulation. Langmuir, 24(21):12469-12473, 2008.

[58] Liangfang Zhang and Steve Granick. Lipid diffusion compared in outer and inner leaflets of planar supported bilayers. The Journal of Chemical Physics, 123(21):211104, 2005.

[59] M. Hetzer, S. Heinz, S. Grage, and T. M. Bayerl. Asymmetric molecular friction in supported phospholipid bilayers revealed by nmr measurements of lipid diffusion. Langmuir, 14(5):982-984, 1998.

[60] Paulo FF Almeida and Winchil LC Vaz. Lateral diffusion in membranes. Handbook of biological physics, 1(C):305-357, 1995.

[61] A. Meyer, R. M. Dimeo, P. M. Gehring, and D. A. Neumann. The high-flux backscattering spectrometer at the nist center for neutron research. Review of Scientific Instruments, 74(5):2759-2777, 2003.

[62] E. Mamontov and K. W. Herwig. A time-of-flight backscattering spectrometer at the spallation neutron source, basis. Review of Scientific Instruments, 82(8), 2011.

[63] R. Mills. Self-diffusion in normal and heavy water in the range 1-45.deg. The Journal of Physical Chemistry, 77(5):685-688, 1973. 
[64] Sow-Hsin Chen. Hydrogen-Bonded Liquids: Quasi-Elastic and Inelastic Neutron Scattering and Molecular Dynamics of Water at Supercooled Temperature, pages 289-332. Springer Netherlands, Dordrecht, 1991.

[65] S. Di Napoli and Z. Gamba. Correlated dynamics of water and amphiphilic molecules in thin newton black films. The Journal of Chemical Physics, 132(7), 2010. 


\section{VITA}

Matthew McCune was born in Kansas City, Missouri. He graduated from Northwest Missouri State University in Maryville, MO in 2010 with a Bachelor of Science degree in Physics under the advisement of Dr. John Shaw and Dr. Himadri Chakraborty. He graduated from the University of Missouri in Columbia, MO in 2012 with a Master of Science degree in Physics. He did his graduate research under

the advisement of Dr. Ioan Kosztin at the University of Missouri, graduating in 2016 with a Ph.D. in Physics. 